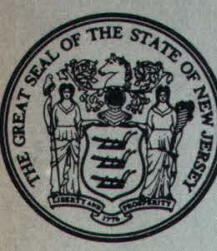

STATE OF NEW JERSEY

HYDROLOGIC STUD'ES

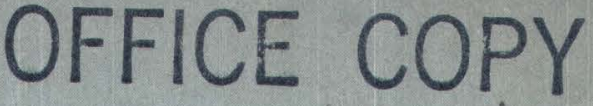

DEPARTMENT OF CONSERVATION AND ECONOMIC DEVELOPMENT

DIVISION OF WATER POLICY AND SUPPLY

RECORDS OF WELLS

and GROUND-WATER QUALITY

in BURLINGTON COUNTY,

NEW JERSEY

WATER RESOURCES CIRCULAR NO. 7

Prepared in cooperation with

United States Department of the Interior

Geological Survey

1962 


\title{
RECORDS OF WELLS AND GROUND-WATER OUALITY IN BURLINGTON COUNTY, NEW JERSEY
}

\author{
A Preliminary Report \\ By \\ F. Eugene Rush \\ Geologist \\ U. S. Geological Survey
}

Prepared by the U. S. Geological Survey in cooperation with the

State of New Jersey 



\section{State of New Jersey}

\section{Department of Conservation}

and Economic Development

H. Mat Adams, Commissioner

\section{Division of Water Policy and Supply \\ George R. Shanklin, Chief Engineer and Acting Director}

Water Policy and Supply Council

Kenneth H. Murray, Chairman ................... Tewksbury

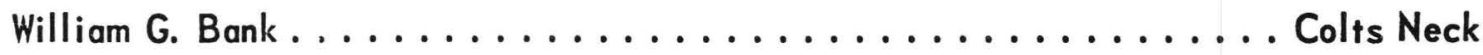

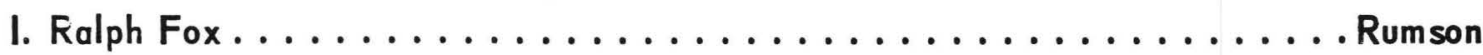

Herman A. Klenner . . . . . . . . . . . . . . . . Rahway Thomas J. Mullen . ..................... Oldwick August C. Schultes...................... Woodbury

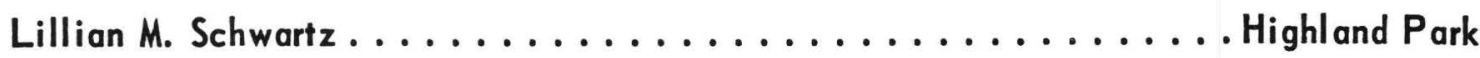
David I. Stepacoff . . . . . . . . . . . . . . . . . . Perth Amboy

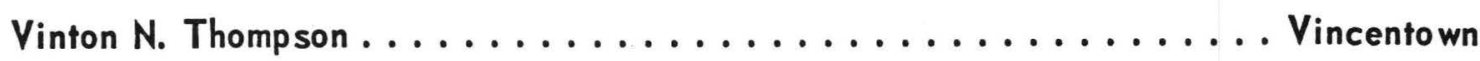
John Wyack, Secretary 



\section{CONTENTS}

\section{Page}

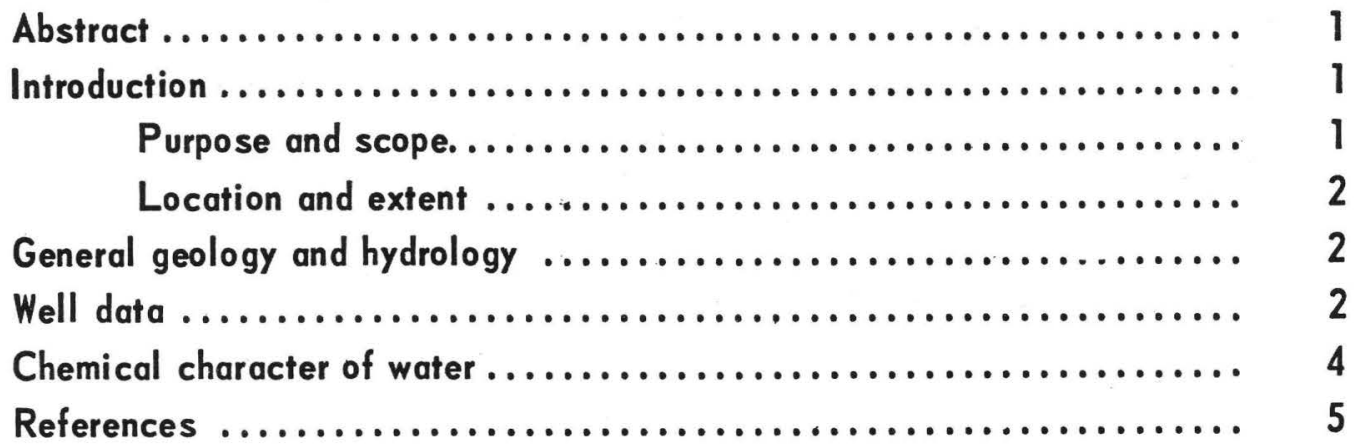

\section{ILLUSTRATIONS}

Figure 1. Map showing location of selected wells in Burlington County, N. J.

Pocket

\section{TABLES}

Page

Table 1. Age, thickness, water-bearing character, and lithology of stratigraphic units in Burlington County, N. J. ............. 3

2 Records of selected wells in Burlington County, N. J. ........ 6

3. Selected drillers' logs of wells in Burlington County,

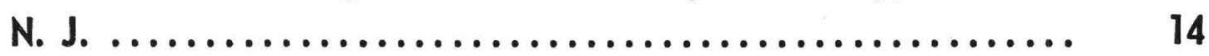

4. Chemical analyses of water from wells in Burlington County,

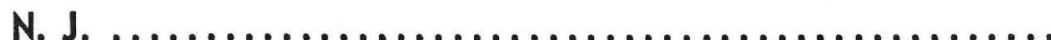





\title{
RECORDS OF WELLS AND GROUND-WATER QUALITY \\ IN BURLINGTON COUNTY, NEW JERSEY \\ by \\ F. Eugene Rush
}

\begin{abstract}
This basic-data report is limited to the presentation of selected drillers' logs and their geologic correlations, well data, and chemical analyses of water from wells in Burlington County, N. J. The county lies within the Coastal Plain province; it is underlain by unconsolidated sediments of Quaternary, Tertiary, and Cretaceous age and at greater depth by much older consolidated rocks.
\end{abstract}

\section{INTRODUCTION}

Purpose and Scope

The investigation of the ground-water resources of Burlington County, New Jersey is part of a state-wide program of water-resources studies being carried out by the U. S. Geological Survey in cooperation with the New Jersey Department of Conservation and Economic Development, Division of Water Policy and Supply. The investigation has been under the general supervision of Allen Sinnott, District Geologist.

This basic-data report is limited to the presentation of selected drillers' logs, well data, and chemical analyses of water from wells in Burlington County. Most of the drillers' logs and the well data were obtained from the files of the New Jersey Geological Survey, Bureau of Topography and Geology, Division of Research and Development, Department of Conservation and Economic Development. The information is released at this time in advance of an interpretive report because of its value to prospective ground-water users in Burlington County. The data in this report form, in part, the basis for a comprehensive, interpretive report on the groundwater resources of Burlington County, now in preparation. 


\section{Location and Extent}

Burlington County is in the southcentral part of New Jersey, extending from the Delaware River on the northwest to Great Bay, an arm of the Atlantic Ocean, on the southeast. The county is bounded by Camden and Atlantic Counties on the southwest and by Mercer, Monmouth, and Ocean Counties on the northeast. (See fig. 1.) Burlington County is the largest county in New Jersey in area, covering 827 square miles. It has a population of 224,499 in the 1960 Federal Census. While the county is principally known for its agriculture, there is considerable manufacturing, particularly along the Delaware River.

\section{GENERAL GEOLOGY AND HYDROLOGY}

Burlington County lies within the Coastal Plain province; it is underlain by layers of sand, silt, and clay of Quaternary, Tertiary, and Cretaceous age. The formation names given to these deposits and the basement crystalline rocks are listed in table 1, which gives their age, thickness, and water-bearing character. The important water-bearing formations in the county are the Raritan and Magothy Formations, the Englishtown Formation, the Wenonah Formation and Mount Laurel Sand, and the Kirkwood Formation and Cohansey Sand.

\section{WELL DATA}

Descriptions of selected wells are given in table 2 . The locations of these wells are shown on figure 1. The drillers' logs in this report have been modified, where necessary, to present a more uniform terminology. The drillers' logs and the author's tentative geologic correlations are given in table 3. 
Table 1. - Age, thickness, water-bearing character, and lithology of stratigraphic units in Burlington County, N. J.

\begin{tabular}{|c|c|c|c|c|c|}
\hline System & Series & Formation & $\begin{array}{c}\text { Thickness } \\
\text { (feet) }\end{array}$ & Water-bearing character & Description \\
\hline Quaternary & Pleistocene & $\begin{array}{l}\text { Cape May Formation } \\
\text { Pensauken Formation }\end{array}$ & $\begin{array}{l}0-40 \\
0-40\end{array}$ & $\begin{array}{l}\text { Variable } \\
\text { Variable }\end{array}$ & $\begin{array}{l}\text { Sand and gravel, local clay } \\
\text { Sand and gravel }\end{array}$ \\
\hline \multirow{4}{*}{ Tertiary } & Pliocene (?) & Cohansey Sand & $0-300$ & Excellent to fair & Sand, medium to coarse, with local ironstone \\
\hline & Miocene & Kirkwood Formation & $0-200$ & Excellent to fair & Sand, fine, some clay seams \\
\hline & Eocene & Manasquan Formation & $0-100$ & Poor & Sand, glauconite, clayey \\
\hline & Paleocene & $\begin{array}{l}\text { Vincentown Formation } \\
\text { Hornerstown Sand }\end{array}$ & $\begin{array}{l}0-100 \\
0-60\end{array}$ & $\begin{array}{l}\text { Good to poor } \\
\text { Poor }\end{array}$ & $\begin{array}{l}\text { Sand, limy, clayey; glauconite } \\
\text { Glauconite sand, with green clay matrix }\end{array}$ \\
\hline Cretaceous & & 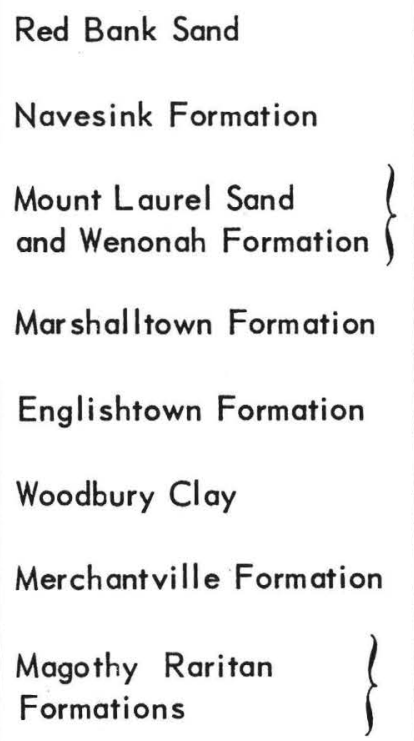 & $\begin{array}{l}0-40 \\
0-40 \\
0-100 \\
0-100 \\
0-100 \\
0-120 \\
0-80 \\
30-800\end{array}$ & $\begin{array}{l}\text { Fair } \\
\text { Poor } \\
\text { Good to poor } \\
\text { Poor } \\
\text { Good to poor } \\
\text { Poor } \\
\text { Poor } \\
\text { Excellent to good }\end{array}$ & $\begin{array}{l}\text { Sand and clay with glauconite sand } \\
\text { Glauconite sand, with, dark gray clay matrix } \\
\text { Sand, clayey, some glauconite; silt } \\
\text { Clay, sandy; glauconite sand } \\
\text { Sand and clay } \\
\text { Clay, black, micaceous } \\
\text { Clay, dark gray, glauconite sand } \\
\text { Clay and sand, alternating }\end{array}$ \\
\hline Early Paleozoic (?) & & Wissahickon Formation & & Poor & Schist or gneiss, commonly weathered \\
\hline
\end{tabular}




\section{CHEMICAL CHARACTER OF WATER}

All ground water contains dissolved mineral matter derived from the sediments, rocks, and soils with which the water has been in contact. The amount and kind of dissolved minerals determine the hardness, corrosiveness, scale-forming properties, and other physical-chemical characteristics of the water. The constituents most likely to occur in objectionable concentrations in ground water are silica $\left(\mathrm{SiO}_{2}\right)$, calcium $(\mathrm{Ca})$, magnesium $(\mathrm{Mg})$, iron $(\mathrm{Fe})$, manganese $(\mathrm{Mn})$, chloride $(\mathrm{Cl})$, and nitrate $\left(\mathrm{NO}_{3}\right)$. In addition, the ground-water properties of importance are dissolved solids, specific electrical conductance, hardness, and $\mathrm{pH}$.

According to Rainwater and Thatcher (1960, p. 141-259), silica in water appears to have little effect on human beings, livestock, fishes, or plants. It is of chief concern to industrial users because it contributes to the formation of hard "boiler scale" on heat-exchange equipment and steam turbine blades. Iron and manganese cause stains on textiles and fixtures if their combined concentration exceeds 0.4 parts per million (ppm); they also cause an unpleasant taste. Chloride is noticeable to the taste at concentrations of above $250 \mathrm{ppm}$ and renders water unpotable at concentrations above 1,000 ppm.

Calcium and magnesium cause most of the hardness of water (Hem, 1959, p. 145). Nitrate, an end product of decomposition of organic matter, when present in concentrations greater than $5 \mathrm{ppm}$ may be indicative of pollution. Water containing more than $44 \mathrm{ppm}$ of nitrate may cause infant cyanosis ("blue-baby disease"), if used in infant-feeding formulas (Maxcy, 1950). The highest concentration of nitrate given on table 4 is 21 ppm, but it generally is less than 1 ppm.

The dissolved-solids content is an index of the total mineralization of the water. Specific conductance is a relative measure of the presence of dissociated ions in solution and thus is also an index of the total mineralization of the water. The specific conductance multiplied by 0.6 to 0.7 qives an approximation of the dissolved-solid content in parts per million.

The property of hardness is difficult to define, in spite of its wide usage (Hem, 1959, p. 145-148). It can be said that hardness represents the soap-consuming power of a water. However, it is commonly expressed in an analysis as an equivalent quantity of calcium carbonate $\left(\mathrm{CaCO}_{3}\right)$. The degree of acidity or alkalinity of water is denoted by $\mathrm{pH}$, on a scale of 1 to 14 . Water having a $\mathrm{pH}$ of 7.0 is neutral; a $\mathrm{pH}$ of less than 7.0 is acidic, and more than 7.0, alkaline. However, in water chemistry a $\mathrm{pH}$ of below 4.5 may indicate free mineral acids, whereas above 4.5 some alkaline ions may be present (Hem, 1959, p. 48). The $\mathrm{pH}$ value of water is primarily of concern to industrial users of water. Chemical analyses of water from wells in Burlington County, N. J. are given in table 4. 


\section{REFERENCES}

Hem, J. D., 1959, Study and interpretation of the chemical characteristics of natural waters: U. S. Geol. Survey Water-Supply Paper 1473, 269 p.

Kummel, Henry B., 1910, Annual report of the State Geologist: New Jersey Geol. Survey Ann. Rept. for 1909, Trenton, N. J., 123 p.

Maxcy, K. F., 1950, Report on the relation of nitrate concentrations in well waters to the occurrence of methemoglobinemia: Nat. Research Council, Bull. Sanitary Eng., p. 265 App. D.

Rainwater, F. H., and Thatcher, L. L., 1960, Methods for collection and analysis of water samples: U. S. Geol. Survey Water-Supply Paper 1454, 301 p.

Richards, Horace C., 1948, Studies on the subsurface geology and paleontology of the Atlantic Coastal Plain: Philadelphia Acad. Nat. Sci. Proc., v. C, p. 39-76.

1954, A new gastropod and other fossils from the Cretaceous of New Jersey: Notulae Naturae, no. 258, Philadelphia Acad. of Nat. Sci. Proc., 5 p.

Smock, John C., 1893, Annual report of the State Geologist: New Jersey Geol. Survey Ann. Rept. for 1892, Trenton, N. J., 367 p.

1894, Annual report of the State Geologist: New Jersey Geol. Survey Ann. Rept. for 1893, Trenton, N. J., 457 p.

-1901, Annual report of the State Geologist: New Jersey Geol. Survey Ann. Rept. for 1900, Trenton, N. J., 231 p. 
Well no.: Numbers correspond to those in figure 2 and tables 3 and 4; Ao indicates chemical analysis given in table 4 , L. indicates drilleris log given in table 3.

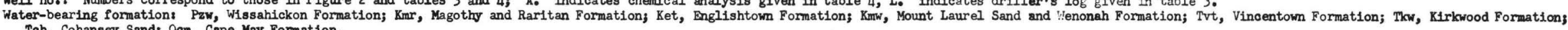
Use : CI, commercial and institutional; D, domestic 3 Ind, Industrial; Irr, Irrigation; O, observation; PS, public supply; U, unused.

\begin{tabular}{|c|c|c|c|c|c|c|c|c|c|c|c|c|c|c|c|c|}
\hline tell & Location & Ormer or name and number & Driller & 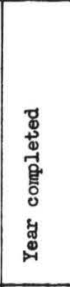 & 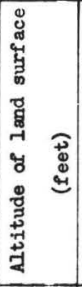 & 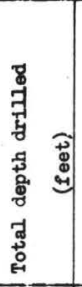 & 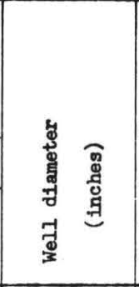 & 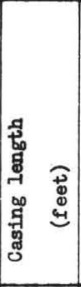 & 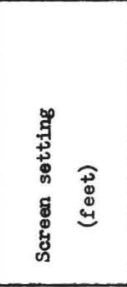 & 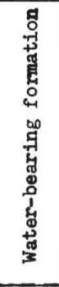 & 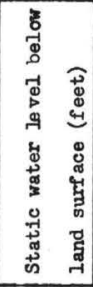 & 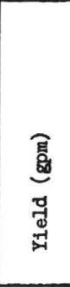 & 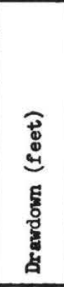 & $\stackrel{\circ}{\square}$ & Remarks & $\begin{array}{l}\text { Woll } \\
\text { no. }\end{array}$ \\
\hline 1 & Bordentown & Bordentown Water Dept. №. 1 & Layne-New York Co., Inc. & 1955 & 89 & 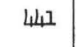 & $16 \& 8$ & 355 & $355-385$ & $\mathrm{Kmr}$ & 86 & 572 & 17 & PS & \multirow{3}{*}{ 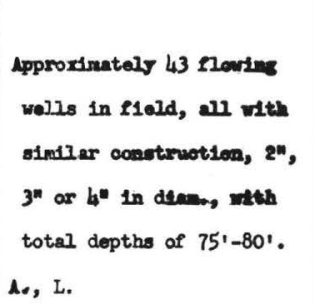 } & 1 \\
\hline 2 & $\begin{array}{l}\text { 2/2 mi. B of U.3. Highway 206, at } \\
\text { Crosswicalcs Creek }\end{array}$ & $\begin{array}{l}\text { Bordentown Water Dept. White } \\
\text { Horse woll field No. A } 40\end{array}$ & & 1941 & 9 & 80 & 3 & 75 & (none) & do. & $\begin{array}{l}\text { (flow- } \\
\text { ing) }\end{array}$ & 25 & & PS & & 2 \\
\hline 3 & Burlengton & Burlington Water Dept. No. 3 & Layne-New York Co., Inc. & 1949 & 9 & 90 & 16 & 64 & $64-85$ & Kner & 10 & 517 & 48 & P8 & & 3 \\
\hline 4 & $\begin{array}{l}\text { I ml. } \mathrm{NE} \text { of Burlington on Burlington } \\
\text { Island }\end{array}$ & $\begin{array}{l}\text { Burlington Water Dept. Test } \\
\text { Well No. } 2 \text { - Island }\end{array}$ & Artesian Well Drilling Co. & 1952 & 19 & 49 & 6 & 40 & 10 & Qcm & I4 & 75 & 8 & 0 & L. & 4 \\
\hline 5 & $\begin{array}{l}1 \text { mi. } N \text { of Burlington on Burlington } \\
\text { Island }\end{array}$ & $\begin{array}{l}\text { Burlington Water Dept. No. } 2 \\
\text { - Island }\end{array}$ & do. & 1952 & 20 & 49 & 17 & 33 & $33-49$ & Qcm & 18 & 760 & 13 & PS & $\begin{array}{l}\text { Concrete casing and screen. } \\
\text { A. }\end{array}$ & 5 \\
\hline 6 & Columbus & Colunbus Water Co. No. 2 & William Stothoff Co. & 1943 & 73 & 260 & 8 & 240 & $240-260$ & $\mathrm{Kmr}$ & 60 & 125 & 40 & PS & A. & 6 \\
\hline$?$ & I ml. N. of Crosswricks & Crosswleks Water Co. No. 1 & do. & 1959 & 30 & 299 & 8 & 269 & $269-299$ & $\mathbf{K m r}$ & 15 & 500 & 44 & PS & A. & 7 \\
\hline 8 & Beverly & Delaware River Water Co. No.15 & A. C. Schultes \& Sons & 1951 & 12 & 59 & 12 & 49 & $47-57$ & $\mathrm{Kmr}$ & 18 & 1200 & 14 & PS & Drilled to bedrock. A. & 8 \\
\hline 9 & Riverside & do. $\quad$ No.17 & do. & 1955 & 21 & 140 & 12 & 97 & $97-118$ & $\mathrm{Kmr}$ & 23 & 483 & 70 & PS & do. & 9 \\
\hline 10 & I mi. SW of Riverside, at Cambridge & $\begin{array}{ll}\text { do. } & \text { No.19 }\end{array}$ & do. & 1959 & 25 & 143 & 12 & 91 & $89-130$ & $\mathrm{Kmr}$ & 山 & 974 & 59 & Ps & do & 10 \\
\hline 11 & Marlton & Evesham Tp. Water Dept. No. 1 & A. G. Durnhey & 1897 & 1215 & 212 & 6 & -- & -- & Ket & 42 & 100 & 36 & PS & A., L. & 11 \\
\hline 12 & Mariton & do. No. 3 & Layno-New York Co., Inc. & 1956 & $8 s$ & 512 & $12 \& 8$ & 369 & $369-389$ & $\operatorname{sm}$ & 91 & 517 & 168 & PS & I. & 12 \\
\hline 13 & Florence & Florence Tp. Water Dept. №.3 & do. & 1949 & 25 & 162 & 16 & 123 & $123-138$ & $\operatorname{sir}$ & 24 & 620 & 34 & PS & Drilled to bedroak. L, L. & 13 \\
\hline 山4 & 2 mi. $S$ of Wrightstown & Fort D1x llo. 1 & Artesian Well Drilling Co. & 1947 & 138 & 980 & $20,16, \& 10$ & 920 & $916-960$ & $\mathrm{Km} r$ & 132 & 800 & 96 & PS & A. & 14 \\
\hline 15 & $1 \mathrm{mi} . \mathrm{S}$ of wrightstown & do. No. 2 & do. & 1941 & 131 & 1051 & do. & 1030 & $1030-1051$ & $\operatorname{xim}$ & 123 & 900 & 61 & PS & A., L. & 25 \\
\hline 16 & $3 \mathrm{mi} . \mathrm{S}$ of Wrightstown & do. No. 4 & Layme-New York Co., Inc. & 1943 & 248 & 1096 & do. & 1056 & $1056-1086$ & $\mathrm{Km}$ & 140 & 700 & 70 & P8 & A., $\mathrm{L}$. & 16 \\
\hline 17 & $3 \mathrm{mi}$. NE of Broms Mills at Hanover Lake & do. No. 11 & William Stothoff Co. & 1943 & 85 & 485 & 6 & 470 & $470-980$ & Ket & 20 & 45 & 193 & PS & L. & 17 \\
\hline 18 & 1 ad. E of Wrightstown & McCuire Air Force Base - A & A. C. Schultes \& Sons & 1953 & 128 & 11100 & $24,16,8<10$ & 992 & $992-1055$ & $\mathbf{I m x}$ & 120 & 913 & 63 & PS & $\begin{array}{l}\text { ropised to weathered bed- } \\
\text { rook. A., L. }\end{array}$ & 18 \\
\hline 19 & 2 mi. E of wrightstown & $-c$ & do. & 1953 & 102 & 1123 & do. & 1036 & $1036-1089$ & $\mathbf{K} \operatorname{mr}$ & 110 & 900 & 45 & PS & $\begin{array}{l}\text { Drilled to weathered bed- } \\
\text { rook. A., L. }\end{array}$ & 19 \\
\hline 20 & 2 m. SB of Wrightstom & $-\mathrm{D}$ & do. & 1953 & 312 & 1120 & do. & 1012 & $1012-1075$ & 1000 & 2110 & 925 & 34 & so & $\begin{array}{l}\text { Drillod to weathered bedt } \\
\text { rock. } 4\end{array}$ & 20 \\
\hline 21 & 3 ad. SW of Modford, at lake Pino & Lalew Woter Co. No. 2 & J. Henxy Robbine & 1950 & 52 & 200 & 6 & 180 & (none) & $\mathrm{K}=$ & 20 & 100 & - & P8 & L, I. & 21 \\
\hline 22 & Lorittona & Lovitt and Sons, Inc. No. 2 & A. C. Schultos \& Sonses & 1955 & 29 & 363 & $20,16,412$ & 225 & $225-354$ & $\sin$ & 22 & Illoh & 65 & 0 & $\begin{array}{l}\text { Sareen blemica } 246-292 \text { and } \\
315-344 . \text {. I. }\end{array}$ & 22 \\
\hline & do. & No. 3 & c. W. Lewenen Co., Inc. & 1959 & 28 & 382 & do. & 203 & 203-303 & $\mathrm{Km}$ & 25 & 1530 & 67 & PB & Soreen blank 238-283. & 23 \\
\hline
\end{tabular}


Table 2.--Records of selected wells in Burlington County, N. J.--Continued

\begin{tabular}{|c|c|c|c|c|c|c|c|c|c|c|c|c|c|c|c|c|}
\hline no. & Location & Onner or name and number & Driller & 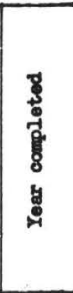 & 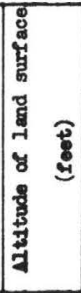 & 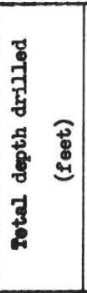 & 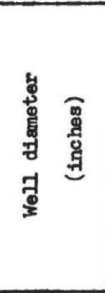 & 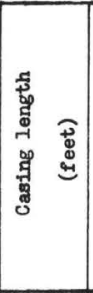 & 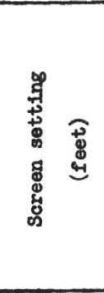 & 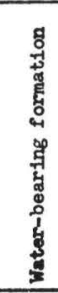 & 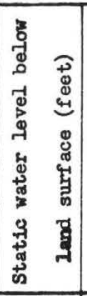 & 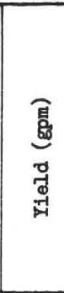 & 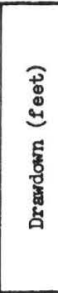 & 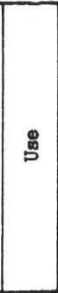 & Bemarks & woll \\
\hline 24 & & 10.4 & A. C. Sehaltas \& soose & 1958 & 28 & 204 & (.). & 177 & $196-279$ & $x=$ & 21 & 1438 & 88 & PS & Bereen alank 226 e59. Lo, $\mathbf{E}$ & 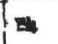 \\
\hline 25 & do. & No. 5 & do. & 1958 & 39 & 269 & do. & 230 & $230-256$ & $\mathbf{K m}$ & 37 & 1122 & 144 & PS & A., L. & 25 \\
\hline 26 & do. & No. 7 & C. W. Lauman Co., Inc. & 1958 & 19 & 306 & do. & 179 & $179-255$ & $\operatorname{smr}$ & 17 & 321 & 56 & PS & A., L. & 26 \\
\hline 27 & do. & No. 12 & A. C. Schultes \& Sons & 1955 & 40 & 526 & - & (none) & (mone) & - & - & -- & - & v & $\begin{array}{l}\text { Abandoned. Drilled as a } \\
\text { test hole. L. }\end{array}$ & 27 \\
\hline 28 & $3 \mathrm{mi} . \mathrm{S}$ of Mount Holly, at Lumberton & $\begin{array}{l}\text { Lumberton Light, Water and } \\
\text { Sewage Co. No. I }\end{array}$ & Artesian Well Drilling Co. & 1928 & 10 & 404 & $10,8,46$ & 360 & $360-401$ & $\mathbf{K} \operatorname{mr}$ & 22 & 100 & - & PS & A., L. & 28 \\
\hline 29 & Maple Shade & Maple Shade Tp. Water Dept. & Uriah white & 1893 & 55 & 375 & - & - & - & $\mathrm{Kmr}$ & $=$ & - & - & PS & Abandoned. L. & 29 \\
\hline 30 & do. & No. 4 & Artesian well Drilling co. & 1955 & 10 & 282 & $20 \& 12$ & 211 & $211-272$ & $\mathrm{Kmr}$ & 19 & 1020 & 42 & PS & L. & 30 \\
\hline 31 & Medf ord & Medford Water Co. No. 3 & Legne-New York Co., Inc. & 1957 & 48 & 590 & $12 \& 8$ & 506 & $506-536$ & $\mathrm{Kmr}$ & 48 & 517 & 86 & PS & L. & 31 \\
\hline 32 & Moorestown & $\begin{array}{l}\text { Moorestomm Tp. Water Dept. } \\
\text { No. } 1\end{array}$ & do. & 1923 & 20 & 497 & $12,8, \& 6$ & 350 & $350-372$ & $\mathrm{Kmr}$ & 28 & 450 & 214 & PS & Drilled to bedrock. A., L. & 32 \\
\hline 33 & Mount Holly & Mount Holly Wetar Co. Ho. 3 & Leopno-Neer Tark Co., Ine. & 1953 & ఋ & 380 & $16 \& 10$ & 316 & $336-346$ & $\mathbf{r m r}$ & 64 & 1200 & 132 & PS & A. & 33 \\
\hline 34 & do. & Mount Holly Water Co. & Thomas B. Harper & 1900 & 20 & 562 & 8 & -- & -- & $\mathrm{Kmr}$ & -- & -- & -- & PS & Abandoned. I. & 34 \\
\hline 35 & Pemberton & Pemberton Water Dept. No. 1 & A. L. Lyons & 1939 & 80 & 206 & $12 \& 8$ & 155 & $155-185$ & $\mathrm{Kmen}$ & 30 & 168 & 51 & PS & A., L. & 35 \\
\hline 36 & Browns Malls & Pemberton Tp. Water Dept. No.2 & Charles Bainbridge & 1947 & 63 & 282 & 8 & 257 & (none) & $\mathrm{Km}$ & 18 & 187 & 27 & PS & A. & 36 \\
\hline 37 & do. & No.3 & Thomas Magee & 1955 & 112 & 303 & 8 & 277 & $277-303$ & $\mathrm{~K}=\mathrm{x}$ & 47 & 250 & 51 & PS & L. & 37 \\
\hline 38 & do. & do. $\quad$ No.4 & Layne-New York Co., Inc. & 1960 & 93 & 402 & $12 \& 8$ & 294 & $294-334$ & $\mathrm{Kmm}$ & 60 & 500 & 11,1 & PS & A., L. & 38 \\
\hline 39 & $4 \mathrm{mi}$. W of Mount Holly at Rancocas Woods & Rancocas Woods Water Co.No.I & Charles L. Mollitar, Inc. & 1954 & 50 & 248 & 6 & 237 & $237-248$ & $\mathrm{Km} x$ & 50 & 150 & 5 & PS & L. & 39 \\
\hline 40 & Palmyra & Riverton-Palmyra Water Co.No.8 & A. C. Schultes \& Sons & 1955 & 13 & 100 & 16 & 64 & $64-84$ & $\mathrm{Kmr}$ & 26 & 503 & 32 & PS & $\begin{array}{l}\text { Drilled to weathered bed- } \\
\text { rock. A.. L. }\end{array}$ & 40 \\
\hline 41 & I $m$. 1 . B of Pemberton at Sunbury Village & Sunbury Village Water Co,No.I & William Stothoff Co. & 2953 & 62 & 198 & 8 & 178 & $178-198$ & $\mathbf{K} \mathrm{mw}$ & 17 & 252 & 119 & PS & $A_{0}^{\text {Lecke }}$ & 42 \\
\hline 42 & $2 \mathrm{mi}$. SE of Bordentown & Norman F. Scattergood & Theodore Ziegenfuss & 1952 & 72 & 362 & 6 & 348 & $348-359$ & Krar & 60 & 60 & 40 & $D$ & Farm use also. L. & 42 \\
\hline 43 & $1 \mathrm{ml}$. $\mathrm{B}$ of Bordentown & Mres. Helen Swayze & William Le Bainbridge & 1952 & 92 & 279 & 6 & 274 & $274-279$ & $\sin$ & 68 & 15 & -- & D & & 43 \\
\hline Lلب & 1 mi. $S$ of Bordentoum on U.S. Hwy. 130 & National Guard Shops & Louls Bainbridge & 1952 & 83 & 230 & 6 & -- & - & $\mathrm{Kmr}$ & - & & -- & CI & L. & 44 \\
\hline 45 & $\begin{array}{l}2 \mathrm{mit} \text {. NE of Jurlington } \\
\text { do. }\end{array}$ & $\begin{array}{l}\text { Hercules Powder Co. No. } 1 \\
\text { do. No.1-test }\end{array}$ & $\begin{array}{c}\text { Layne-New York Co., Inc. } \\
\text { do. }\end{array}$ & $\begin{array}{l}1946 \\
1945\end{array}$ & 22 & 1,6 & 10 & 105 & $105-135$ & $\mathrm{Km} r$ & $\begin{array}{r}10 \\
5\end{array}$ & 520 & 21 & CI & Drillled to bedrock. A.,L. & 45 \\
\hline 47 & do. & do. No.3-test & do. & 1945 & 16 & 121 & 3 & 94 & $94-104$ & $\mathbf{K m} \mathbf{r}$ & 山 & $\ldots$ & - & 0 & do. A. & 47 \\
\hline 48 & 2 mi. SW of Burlington on U.S. HWy. 130 & Edgar B. Heisler & Charles L. Mollitor, Inc. & 1958 & 30 & 2215 & 10 & 185 & $185-215$ & $\mathbf{x} \mathbf{m r}$ & 33 & 600 & 82 & $\operatorname{Irx}$ & L. & 48 \\
\hline 49 & I md. SW of Burlington on U.S. Hwy. 130 & Carroll Murphy & do. & 1950 & 35 & 83 & 8 & 64 & $64-82$ & $\operatorname{rm}$ & 15 & 250 & 45 & $\operatorname{Irr}$ & L. & $\omega \theta$ \\
\hline 50 & $2 \mathrm{mi} . \mathrm{SE}$ of Burlington & Masonic Howe & Amorican Well Drilling co. & 1921 & 79 & 395 & 8 & 174 & $174-194$ & $r$ & 65 & 150 & 45 & v & A., L. & 50 \\
\hline 51 & $3 \mathrm{mi} . \mathrm{E}$ of Burlington & Willien Donald & Charles L. Mollitor, Inc. & 2955 & 42 & س & 10 & 72 & $72-106$ & $\operatorname{rar}$ & 26 & 275 & 24 & Ixs & L. & 51 \\
\hline 52 & $3 \mathrm{mt}$. SE of Burlington on Oxmead Rd. & Russell H. Deacon & W. R. Cladr & 1958 & 62 & 208 & 4 & 203 & $203-208$ & $\mathbf{x}=\mathbf{x}$ & 62 & 30 & -- & D & & 52 \\
\hline 53 & $3 \mathrm{mil}$. SE of Burlington & Roy F. Main & E. Weik & 1959 & 67 & 215 & 4 & 207 & $207-215$ & $\mathbf{r m r}$ & 60 & 30 & 5 & D & & 53 \\
\hline 54 & $\begin{array}{l}3 \mathrm{mi} . \text { SE of Burlington on Oxonead Rd. } \\
2 \mathrm{ml} \text {. SW of Burlington }\end{array}$ & $\begin{array}{l}\text { Chorry Hill Inn Farm No. } 1 \\
\text { Frank Pino }\end{array}$ & $\begin{array}{l}\text { Charles L.Mollitor, Inc. } \\
\text { Edvard Robbins }\end{array}$ & $\begin{array}{l}1950 \\
1950\end{array}$ & $\begin{array}{l}72 \\
63\end{array}$ & $\begin{array}{l}208 \\
228\end{array}$ & $\begin{array}{c}6 \\
8 \& 6\end{array}$ & $\begin{array}{l}199 \\
208\end{array}$ & $\begin{array}{l}199-206 \\
206-228\end{array}$ & $\mathbf{x}=$ & $\begin{array}{l}82 \\
60\end{array}$ & $\begin{array}{r}10 \\
300\end{array}$ & 36 & D & $\begin{array}{l}\text { Tans wae also. L. } \\
\text { Puing expacity is } 400 \text { gpm.L. }\end{array}$ & $\begin{array}{l}54 \\
55\end{array}$ \\
\hline
\end{tabular}


Table 2.--Records of selected wells in Burlington County, N. J.--Contimued

\begin{tabular}{|c|c|c|c|c|c|c|c|c|c|c|c|c|c|c|c|c|}
\hline WeIl & Location & Owner or name and number & Driller & 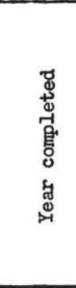 & 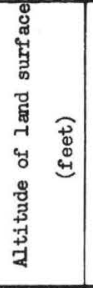 & 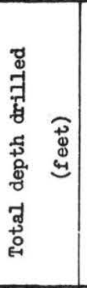 & 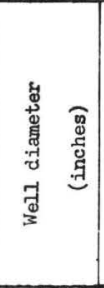 & 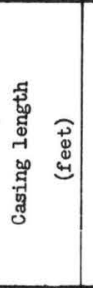 & 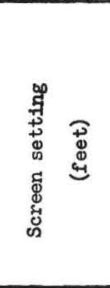 & 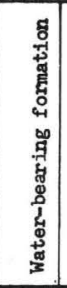 & 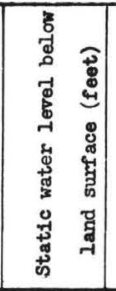 & 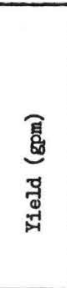 & 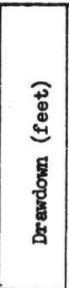 & $\Phi$ & Remarks & well \\
\hline 56 & 1 mi. SW of Florence & National Cypsum Co. No. 2 & Layne-New York Co., Inc. & 1955 & 22 & 157 & $12 \& 8$ & 113 & $113-123$ & $\mathbf{K} m \mathbf{r}$ & 21 & 183 & 21 & Ind & Drilled to bedrock. L. & 56 \\
\hline 57 & $2 \mathrm{mi}$. SW of Burlington on U.St Hary. 130 & Mechanics National Bank No. 2 & Charles L. Mollitor, Inc. & 1958 & 35 & 209 & 4 & 189 & 189-209 & $\mathrm{Kmr}$ & 30 & 180 & 27 & CI & $\begin{array}{l}\text { Air conditioning recharge } \\
\text { well. }\end{array}$ & 57 \\
\hline 58 & 1 mi. $S$ of Burlington & Charles Heal, Jr. & do. & 1955 & 50 & 80 & 8 & 59 & $59-80$ & $\mathrm{Kmr}$ & 36 & 400 & 50 & $\operatorname{Irr}$ & $\begin{array}{l}\text { Water purped into irriga- } \\
\text { tion pond. } \mathbf{L} \text {. }\end{array}$ & 58 \\
\hline 59 & $2 \mathrm{mi} . \mathrm{SW}$ of Burlington & Anthony Cannuli & Edward Robbins & 1950 & 66 & 173 & 6 & 153 & $153-173$ & $\mathrm{Kmr}$ & 55 & 200 & 30 & $\operatorname{Ir} r$ & $\begin{array}{l}\text { Water purped into irriga- } \\
\text { tion pond. }\end{array}$ & 59 \\
\hline 60 & 2 mi. SW of Burlinston on U.S. Hory. 130 & Albert Heisler & do. & 1950 & 46 & 252 & 8 & 212 & $212-252$ & $\mathrm{~K} n \mathrm{n}$ & 41 & 500 & 42 & $\operatorname{Irr}$ & Pump capacity is $500 \mathrm{gpm}$. & 60 \\
\hline 61 & II mi. $S$ of Chatsworth, at Harrisville & Harrisville well & -- & 1866 & 15 & 375 & 8 & --- & -- & Ther & (flowing) & --- & - & o & $\begin{array}{l}\text { Well is still flowing. } \\
\text { A., } L \text {. }\end{array}$ & 61 \\
\hline 62 & $2 \mathrm{mt}$. NE of Bordentown & N.J. State Reformatory No. 4 & Layne-New York Co., Inc. & 1951 & 97 & 454 & $12 \& 8$ & 357 & $357-387$ & $\mathrm{~K} n \mathrm{rr}$ & 86 & 503 & 36 & U & A., L. & 62 \\
\hline 63 & $4 \mathrm{mfl}$. SE of Bordentown, at Chesterfield & H. W. Eriel & Howard Farmer & 1959 & 93 & 272 & 4 & 266 & $266-272$ & $\mathrm{Kmr}$ & 72 & 50 & 10 & D & & 63 \\
\hline 64 & $\begin{array}{l}1 \text { mi. SW of Chesterfield, on Whitepine } \\
\text { Rd. }\end{array}$ & Ralph J. DeMarco & Louis Bainbridge & 1958 & 82 & 393 & 4 & 388 & $388-392$ & $\mathrm{~K} \min$ & 75 & 4 & 45 & D & & 64 \\
\hline 65 & 3 mi. SE of Bordentown, on 01d York Rd. & Ernest Liptak & Greenhalgh \& Kaye & 1957 & 114 & 214 & 4 & 208 & 208-214 & $\mathrm{Kmr}$ & 100 & 10 & 20 & D & & 65 \\
\hline 66 & 2 mi. If of Wrightstonn & Jacob Dana Packing Co. No. 3 & do. & 1958 & 208 & 276 & 6 & 255 & $255-276$ & Let & 100 & 60 & 80 & CI & L. & 66 \\
\hline 67 & $7 \mathrm{mi}$. N of Wrightstown, on Ellisdale Rd. & Francis D. Gray & Louis Bainbridge & 1960 & 92 & 329 & 6 & 319 & $319-325$ & $\mathrm{Kmar}$ & 75 & 40 & 5 & D & $\begin{array}{l}\text { Used for swimming pool } \\
\text { also. A, L. }\end{array}$ & 67 \\
\hline 68 & 8 mi. $N$ of Wrightstorm, on Ellisdale Rd. & Johm Marincas & Greenhalgh \& Kaye & 1954 & 97 & 208 & 6 & 204 & $204-208$ & $\mathrm{Kmr}$ & 80 & 25 & 69 & D & I. & 68 \\
\hline 69 & 4 mi. N of Wrightstown & Joseph S. Kucowski & do. & 1957 & 104 & 149 & 6 & 143 & $143-1149$ & Ket & 38 & 10 & 7 & D & & 69 \\
\hline 70 & 2 mi. $\mathbb{N E}$ of Riverton & Hoeganaes Sponge Iron Co. & Layne-New York Co., Inc. & 1951 & 35 & 136 & 8 & 119 & $119-134$ & $\mathrm{Kmr}$ & 16 & 326 & 68 & CI & Punm capacity is $50 \mathrm{gpm}$. I. & 70 \\
\hline 7 & $\begin{array}{l}3 \mathrm{mi} \text {. NE of Mount Holly, on State Route } \\
537\end{array}$ & Eastampton School & Charles L. Mollitor, Inc. & 1957 & 73 & 417 & -- & 407 & $407-427$ & $\mathrm{~K} \pi \mathrm{r}$ & 70 & 75 & 30 & U & I. & 7 \\
\hline 72 & $\begin{array}{l}1 \text { mi. NE of Mount Holly, on North Pear- } \\
\text { berton Rd. }\end{array}$ & J. A. Methrin & do. & 2953 & 39 & 364 & 6 & 352 & $352-363$ & rom & 63 & 30 & 25 & D & I. & 72 \\
\hline 73 & 1 mi. of Marlton, on Cropwell Rd. & $\begin{array}{l}\text { Byron T. Roberts Farms Inc. } \\
\text { Mo. } 2\end{array}$ & A. C. Schultes \& Sons & 2957 & 93 & 406 & $18 \& 8$ & 322 & $322-375$ & - & 100 & 750 & 80 & $\operatorname{Irr}$ & $\begin{array}{l}\text { Screen blank 348-359. } \\
\text { A., L. }\end{array}$ & 73 \\
\hline 74 & 12 ad. W of Mariton, on 01d Marltea Pike & David D. Griseom & J. Henty Robbins & 1952 & 9 & 340 & b & 320 & 3000 & 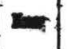 & 100 & $\omega$ & - & D & I. & 76 \\
\hline 75 & $2 \mathrm{mi}, \mathrm{SE}$ of Marlton & $\begin{array}{l}\text { U.S. Army N1ke Base, control } \\
\text { area well }\end{array}$ & Edward Robbins, $\mathrm{sr}$. & 1954 & 84 & 258 & 8 & 138 & $138-158$ & $\mathrm{kmm}$ & 42 & 200 & 43 & o & & 75 \\
\hline 76 & 1 mi. SE of Beveriy, on U.S. Highwas 130 & Earl R. Blyler & Charles I. Mollitor, Inc. & 1950 & 39 & 117 & 10 & 90 & 90-114 & $x$ & $\mathbf{1 4}$ & 575 & 19 & $\operatorname{Irr}$ & L. & 76 \\
\hline 77 & 1 mi. SE of Beverly, on Cooper St. & John w. Heal & do. & 2951 & 36 & 235 & 10 & 207 & $107-135$ & $\operatorname{sor}$ & 18 & 500 & 89 & $\operatorname{Irr}$ & A. & 77 \\
\hline 78 & I mi. SW of Beveriy, at Cambridge & Amico Sand and Gravel co. & do. & 1957 & 15 & 75 & 6 & 27 & $27-37$ & $\mathbf{r m}$ & 7 & 120 & 11 & CI & L. & 78 \\
\hline 79 & 1 mi. $S$ of Riverside, on U.s. Hhy 130 & Hullide Farms No. 3 & A. C. Schultes \& Sons & 1956 & 20 & 112 & 12 & 90 & $90-106$ & $\mathrm{Rm}$ & 6 & 4 & 68 & CI & & 79 \\
\hline 80 & $1 \mathrm{mI}$. SB of Reverside, on $0 . s$. Hory 130 & Holy Cross High School & Charles L. Mollitor, Inc. & 1958 & 82 & 174 & 10 & 154 & $154-174$ & $\mathbf{x m r}$ & 55 & 185 & 20 & Irr & L. & 80 \\
\hline 81 & I at. E of Riverside, on U.S. Hovy 130 & Holiday Lake & do. & 1958 & 25 & 198 & 8 & 188 & $188-198$ & $\mathrm{Kmr}$ & 25 & 180 & - & CI & L. & 81 \\
\hline
\end{tabular}


Table 26--Records of selected wells in Burlington County, N. J.--Continued

\begin{tabular}{|c|c|c|c|c|c|c|c|c|c|c|c|c|c|c|c|c|}
\hline werl & Location & Omer or name and number & Driller & 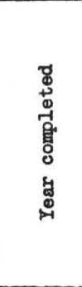 & 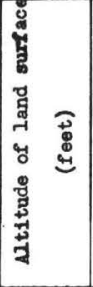 & 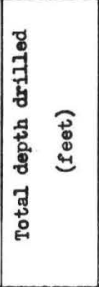 & 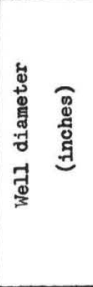 & 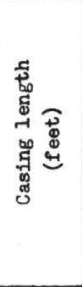 & 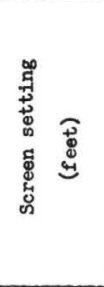 & 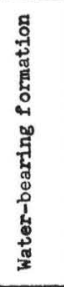 & 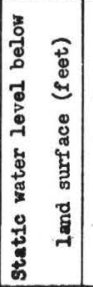 & 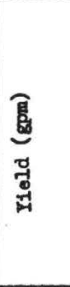 & 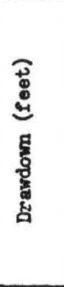 & $\stackrel{\Phi}{\square}$ & Remarks & $\begin{array}{l}\text { Well } \\
\text { no. }\end{array}$ \\
\hline 82 & 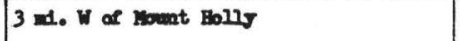 & Nifred Thomes & ertesion Well Drfiling Co. & 1954 & 34 & 75 & 4 & 70 & $70-74$ & Kot & 33 & 4 & 29 & D & & ex \\
\hline 83 & $4 \mathrm{ml}$. SW of Mount Holly & Fred Worth & Edward Robbins, Sr. & 1949 & 30 & 317 & 8 & 277 & $277-297$ & $\mathbf{K m r}$ & 23 & 200 & 97 & $\operatorname{Irr}$ & Prump capacits is 200 epm. & 83 \\
\hline 84 & $3 \mathrm{ml} . \mathrm{NE}$ of Modford & Charles H. Clark & Charles I. Mollitar, Inc. & 1956 & 51 & 191 & 4 & 184 & $184-190$ & Ket & 25 & 35 & 20 & $\mathrm{D}$ & & 84 \\
\hline 85 & Moumt Holly & Plasticon Corp. & do. & 1955 & 45 & 107 & 6 & 97 & $97-107$ & Ret & 40 & 75 & 5 & cI & & 85 \\
\hline 86 & $3 \mathrm{mi}$. E. of Burlingtion & John and N1ck Cannuli & do. & 1955 & 41 & 93 & 10 & 72 & $72-92$ & $\mathrm{xm}$ & 29 & 250 & 30 & $\operatorname{Irr}$ & Puap capacity is $500 \mathrm{gpm}$. & 86 \\
\hline 87 & do. & Janes Workman & Edward Robbins, Sr. & 1952 & 41 & 194 & 10 & 174 & $174-194$ & $\mathrm{Kmr}$ & 52 & 800 & 32 & $\operatorname{Irr}$ & do. & 87 \\
\hline 88 & $\begin{array}{l}3 \mathrm{mi} \text {. W of Wrightstown on State } \\
\text { Route } 54.5\end{array}$ & John H. Cook & Charles I. Mollitor, Inc. & 1950 & 1115 & 148 & 6 & 136 & $136-148$ & Set & 36 & 50 & 4 & D & $\begin{array}{l}\text { Water temperature is } \\
549 \text { \%. L. }\end{array}$ & 88 \\
\hline 89 & $2 \mathrm{~mL} . \mathrm{S}$ of Bordentorn on U.S. Hhy 206 & Turnpike Junction Ind. Park & A. C. Schultes \& Sons & 1958 & 60 & 340 & 10 & 293 & $293-329$ & $\mathrm{Km}$ & 55 & 402 & 31 & $\bar{\sigma}$ & $\begin{array}{l}\text { Screen blank 307-318. } \\
\text { Undereloped induestriel } \\
\text { park. A., L. }\end{array}$ & 89 \\
\hline 90 & 4 mi. $S$ of Bordentorm & Franklin Tallman & Charles L. Mollitor, Inc. & 1949 & 82 & 195 & 6 & 184 & $184-195$ & $\mathbf{K m}$ & 75 & 38 & 5 & $\mathrm{D}$ & Farm use also. L. & 90 \\
\hline 91 & $3 \mathrm{ml} . \mathrm{S}$ of Bordentowm & Ed Phares & Louis Bainbirdge & 1949 & 92 & 505 & 6 & 364 & $364-370$ & $\mathrm{x}_{\mathrm{mr}}$ & 125 & -- & - & $\mathrm{D}$ & $\begin{array}{l}\text { Farm use also. Pump } \\
\text { capacity is } 20 \mathrm{gpm} . \mathrm{L} \text {. }\end{array}$ & 91 \\
\hline 92 & do. & George H. Preidel & Edward Robbins, Sr. & 1952 & 102 & 215 & 6 & 205 & $205-215$ & $\mathrm{xm}$ & 100 & 50 & 5 & $\mathrm{D}$ & Farm use also. & 92 \\
\hline 93 & do. & M. P. Woodward & W. R. Glair & -- & 72 & 239 & 3 & 234 & $234-239$ & Inror & 54 & 20 & - & D & & 93 \\
\hline 94 & $4 \mathrm{mi} . \mathrm{SE}$ of Bordentowm & $\begin{array}{l}\text { North Burlington Co. Regional } \\
\text { Jr.-Sr. High School }\end{array}$ & Russell D' Agostino & 1959 & 83 & 350 & $8 \& 6$ & 290 & $290-310$ & $\mathrm{Km}$ & 85 & 200 & 35 & cI & Water contains iroa. I. & 94 \\
\hline 95 & $6 \mathrm{mi} . \mathrm{E}$ of Burlington & Ralph R. Parker & W. R. Clair & 1959 & 62 & 380 & 4 & 370 & $370-380$ & $\mathrm{x}_{\operatorname{mr}}$ & 50 & 50 & 5 & $\mathrm{D}$ & Farm use also. L. & 95 \\
\hline 96 & $5 \mathrm{mil} . \mathrm{E}$ of Burlington & Franklin Wainwright & do. & $\cdots$ & 62 & 209 & 3 & 204 & $204-209$ & $\mathbf{r m}$ & 42 & 20 & - & $\mathrm{D}$ & & 96 \\
\hline 97 & $1 \mathrm{mi}$. $\mathrm{N}$ of Medford on Church Rd. & Peter Martin & John S. Wrobel & 1949 & 62 & 126 & 3 & 123 & $123-126$ & $\mathbf{x m}$ & थ2 & 10 & 2 & $\mathbf{D}$ & A., $\mathbf{l}$. & 97 \\
\hline 98 & 2 mi. NW of Medford on Church Rd. & William E. Jolmson, Jr. & Charles L. Mollitor, Inc. & 1950 & 79 & 440 & $8 \& 6$ & 420 & $420-440$ & $\cos$ & 65 & 200 & 45 & $\operatorname{Irx}$ & L. & 98 \\
\hline 99 & $\begin{array}{l}\text { I mi. S of Medford Lakes on Tuckerton } \\
\text { Rd. }\end{array}$ & George Aaron & J. Henry Robbins & 1952 & 2116 & 340 & 4 & 320 & (none) & $\sin$ & 50 & 50 & - & D & L. & 99 \\
\hline 100 & $\begin{array}{l}3 \mathrm{ml} . \mathrm{SW} \text { of Medford Lakes, at Gen- } \\
\text { tennial Lake }\end{array}$ & William G. Freeman & do. & 1955 & 65 & 275 & 4 & 260 & (none) & $x=$ & 20 & 40 & - & D & L. & 100 \\
\hline 102 & $\begin{array}{l}2 \text { mi. W of Modford Lakes, at Taunton } \\
\text { Lake }\end{array}$ & Robert M. Dickson & do. & 1951 & 66 & 242 & 4 & 222 & (none) & $\operatorname{rnar}$ & 20 & 100 & - & D & & 101 \\
\hline 102 & I ml SE of Maple Shade on State Hry. 73 & Albert E. Crisp & Haines and Moore & 1951 & 62 & 197 & 4 & 290 & $190-196$ & $\operatorname{Kar}$ & 58 & 20 & 5 & D & $\begin{array}{l}\text { Watar temperature is } \\
56{ }^{\circ} \text {. Water conteins } \\
\text { Iron. L. }\end{array}$ & 202 \\
\hline 103 & State HIghway 38, Maple Shade & Savar Corporation & Charles L. Mollitor, Inc. & 1955 & 62 & 166 & 6 & 155 & $255-166$ & $=$ & 65 & 120 & 20 & CI & $\begin{array}{l}\text { Water used at a drivo-in } \\
\text { theater. }\end{array}$ & 103 \\
\hline 104 & 3 mí. E ar woorestown, on Harne thry. & wiziliam Haines, Jr. & Thowas C. Magee, Jr. & 1954 & 52 & 55 & 4 & 47 & $47-53$ & rot & 21 & 10 & 20 & D & A., I. & ioh \\
\hline 105 & $2 \mathrm{mi}$. SB of Moorestonen & Roy w. Conrow & J. Henry Robbins & 1952 & 37 & 205 & 6. & 195 & $295-205$ & $\mathbf{r}$ & 60 & 50 & - & D & $\begin{array}{l}\text { Water tepperatase is } \\
56 \% \text { T. In }\end{array}$ & 105 \\
\hline 106 & 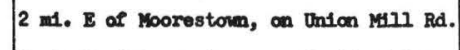 & Joln MeNicholas & Charles L. Mollitox, Inc. & 2955 & 30 & 290 & 4 & 284 & $284-290$ & $x=$ & 22 & 25 & 3 & D & & 106 \\
\hline 107 & $3 \mathrm{mi} . \mathrm{E}$ of Moorestomn, on Hartford Rd. & Thomas P. MeGinnis & J. Henry Robbins & 1952 & 46 & 276 & 4 & 256 & $256-276$ & $x$ & 50 & 50 & - & $\mathrm{D}$ & I. & 207 \\
\hline
\end{tabular}




\begin{tabular}{|c|c|c|c|c|c|c|c|c|c|c|c|c|c|c|c|c|}
\hline nell & Location & Onnor or newe and number & Driller & 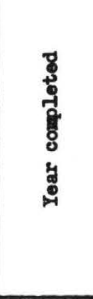 & 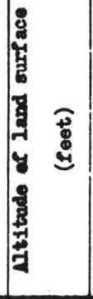 & 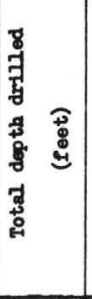 & 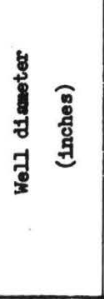 & 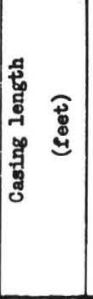 & 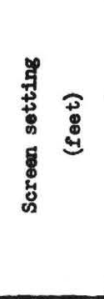 & 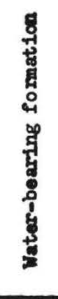 & 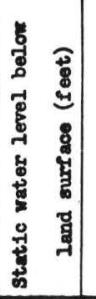 & 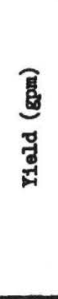 & 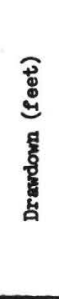 & $\stackrel{8}{\square}$ & Reanikes & noll \\
\hline 108 & $3 \mathrm{mi}$. If of moorestom & Dondinis Trescano & Ores Hauser & 2954 & 28 & 196 & 3 & 182 & 182-192 & $x$ & 48 & 8 & - & D & Water teperaturese is & 108 \\
\hline 309 & Ior. & New Jersey Turmpike & do. & 2951 & 50 & 230 & 8 & 192 & $192-222$ & $\operatorname{Kmr}$ & 48 & 970 & 140 & cI & $\begin{array}{l}\text { beed for atr condition- } \\
\text { ing aleo. }\end{array}$ & 109 \\
\hline$m 0$ & 4 d. E of Moorostom & J. S. Coverly & Charles L. Bollitor, Inc. & 1954 & 32 & 250 & 4 & 244 & $24 \sqrt{4-250}$ & $\mathbf{r}$ & 30 & 50 & 10 & D & & 210 \\
\hline בוב & 3 mi. wadrord, on Hartford Rd. & Eva Diamond & John S. Wrobel & 1951 & 73 & 202 & 4 & 190 & 190-200 & Ket & 48 & 10 & 6 & D & L. & m \\
\hline 132 . & $4 \mathrm{mi}$. SE of Moorestorm, on Hartiford Rd. & Anthong Palumbo & J. Henry Robbins & 1949 & 66 & 348 & 4 & 328 & $328-348$ & $\mathrm{Kmr}$ & 60 & 50 & - & D & & 122 \\
\hline 123 & $4 \mathrm{mi}$. SE of Moorestom & Samuel Dickter & Charles L. Mollitor, Inc. & 1957 & 50 & 300 & 4 & 292 & $292-300$ & $\mathrm{Kmr}$ & 60 & 40 & 5 & $\mathrm{D}$ & I. & 113 \\
\hline 124 & do. & Gertrude Froeman & do. & 1953 & 38 & 262 & 4 & 256 & $256-262$ & $\sin$ & 33 & 15 & 8 & D & & Ш \\
\hline 125 & $\begin{array}{l}4 \mathrm{mil} \text {. W of Mount Holly, at Rancocos } \\
\text { Woods }\end{array}$ & Walter H. Kopp & do. & 1952 & 43 & 237 & 6 & 227 & $227-237$ & $\sin$ & 41 & 20 & 46 & D & & 215 \\
\hline 126 & 2 mi. SE of Maple Shade, on Church Rd. & Charles J. Ruhle & J. Henry Robbins & 1951 & 57 & 240 & 4 & 230 & $230-240$ & $\mathrm{Kmr}$ & 60 & 50 & - & D & L. & 116 \\
\hline 217 & $\begin{array}{l}2 \text { mi. SE of Maple Shade, on State } \\
\text { Highway } 73\end{array}$ & Felix Oliveto & Charles L. Mollitor, Inc. & 1953 & 63 & 223 & 4 & 216 & 216-22? & Kmar & 55 & 60 & 10 & D & L. & 117 \\
\hline 278 & do. & Ralph Vasturo & J. Henry Robbins & 1950 & 68 & 119 & 6 & 100 & (none) & Xet & 25 & 10 & - & D & A. & 128 \\
\hline 119 & $3 \mathrm{ml}$. E. of Moorestom, on Hartfond Rd. & John D. Hallet & Charles L. Mollitor, Inc. & 1956 & 32 & 244 & 4 & 238 & $238-244$ & $\sin$ & 35 & 30 & 20 & D & & 119 \\
\hline 120 & $3 \mathrm{mi}$. E of Moorestom, at Masonville & William Breur & do. & 1958 & 78 & 272 & 4 & 266 & $266-272$ & $\ln$ & 90 & 40 & 10 & $\mathrm{D}$ & I. & 120 \\
\hline 122 & 2 mi. w of Marlton, on State thy 73 & W. R. Fought & do. & 1953 & 85 & 284 & 4 & 276 & 276-281 & $\operatorname{Knar}$ & 89 & 15 & 10 & D & & 121 \\
\hline 122 & $2 \mathrm{ml}$. $\mathrm{NE}$ of Marlton & Joseph E. Rudderow & do. & 1954 & 48 & 457 & $8 \& 6$ & Lلب & $441-457$ & $\operatorname{Km} x$ & 56 & 450 & 125 & $\operatorname{Irx}$ & $\begin{array}{l}\text { Pump copacity is } 450 \\
\text { gpm. L. }\end{array}$ & 122 \\
\hline 123 & $3 \mathrm{ml}$. $\mathrm{NB}$ af Mariton & do. & William C. Barr & $\begin{array}{l}\text { before } \\
1898\end{array}$ & 82 & 306 & 3 & - & - & - & 50 & - & - & D & $\begin{array}{l}\text { Mrs. Samuel Shroeve was } \\
\text { the owner at tho tive } \\
\text { the wall was drilled. }\end{array}$ & 123 \\
\hline 124 & do. & Walter E. Cotter, Jr. & Edward Robbins, Sr. & 1955 & 77 & 384 & 4 & 379 & $379-384$ & Knrr & 70 & 60 & 10 & D & a. & 124 \\
\hline 125 & $4 \mathrm{mi}$. SB of Moorestorm, on Hartford Rd. & Saul Grossmen & Charles L. Mollitor & 1951 & 40 & 105 & 4 & 98 & $98-105$ & Xet & 22 & 10 & 18 & D & A. & 125 \\
\hline 126 & New Albany Rd., Moorestorm & P1ttsburgh Plate Glass Co. & Layne-New York Co., Inc. & 1947 & 65 & 318 & $16 \& 10$ & 263 & 263-283 & $\sin$ & 65 & 517 & 94 & Ind & A., I. & 126 \\
\hline 127 & Mount Bolly & $\begin{array}{l}\text { Hollypiord Ioe \& Cold Starage } \\
\text { No. } 1\end{array}$ & do. & 1946 & 10 & 500 & 10 & 470 & $470-500$ & $\mathrm{Kmr}$ & 4 & 350 & 29 & CI & & 127 \\
\hline 128 & do. & Dunlep's Carpet Workcs & c. G. Orcutt & $\begin{array}{l}\text { before } \\
1893\end{array}$ & 12 & 675 & - & -- & - & - & - & - & - & $\mathbf{0}$ & Wall is abendonod. I. & 128 \\
\hline 129 & do. & Nowo Market & Charles I. Mollitor, Inc. & 1956 & 62 & 467 & 8 & 456 & $456-467$ & Kum & 75 & 225 & 10 & cI & 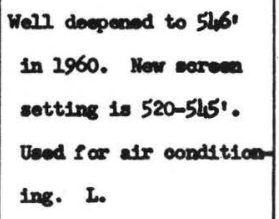 & 129 \\
\hline 1300 & 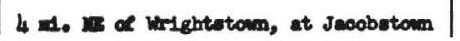 & Jecobstonen Beptist Church & Andrew Whit to & 1951 & 175 & $\mathrm{UH}_{4} \mathrm{O}$ & 4 & 136 & $136-140$ & $\mathbf{n}=$ & 50 & 25 & - & CI & I. & 230 \\
\hline
\end{tabular}


Table 2.--Records of selected wells in Burlingtion County, N. J.--Continued

\begin{tabular}{|c|c|c|c|c|c|c|c|c|c|c|c|c|c|c|c|c|}
\hline $\begin{aligned} \text { Well } \\
\text { no. }\end{aligned}$ & Location & Omer or name and number & Driller & 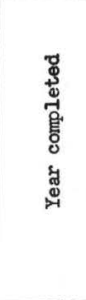 & 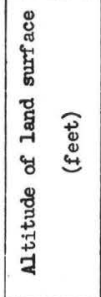 & 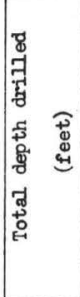 & 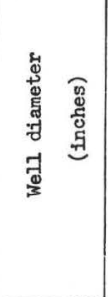 & 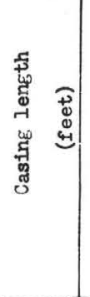 & 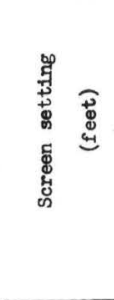 & 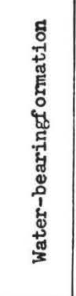 & 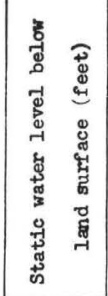 & 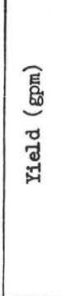 & 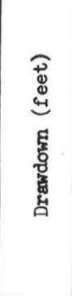 & $\stackrel{\square}{\square}$ & Remarks & noll \\
\hline 131 & $\begin{array}{l}4 \text { mi. NE of Wrightstom, at Ocean co. } \\
\text { line. }\end{array}$ & $\begin{array}{l}\text { Jersey Oantral Power \& } \\
\text { Light co. }\end{array}$ & areenhelgh \& Iaje & 2956 & 88 & 121 & 6 & 110 & $110-121$ & $\mathrm{Km}$ & 3 & 30 & 105 & CI & A., L. & 131 \\
\hline 132 & $5 \mathrm{mi} . \mathrm{N}$ of Wrightstown & Edrard H. Cancarz & do. & 1954 & 121 & 157 & 4 & 148 & $\mu_{18-152}$ & Ket & 35 & 10 & 17 & $\mathrm{D}$ & L. & 132 \\
\hline 233 & $6 \mathrm{mi} . \mathrm{N}$ of Wrightstom & Stanley Horner, Jr. & do. & 1958 & 118 & 254 & 4 & 151 & 151-154 & Ket & 40 & 15 & 25 & $\mathrm{D}$ & & 133 \\
\hline 134 & wriehtstown & Spartan Village, Inc. & $\begin{array}{l}\text { Louis Bainbridge and } \\
\text { Charles L. Mollitor, Inc. }\end{array}$ & 1955 & 154 & 395 & 6 & 128 & $228-150$ & Kom & 26 & 40 & 19 & $\mathrm{v}$ & L. & 134 \\
\hline 135 & $5 \mathrm{mi} . \mathrm{N}$ of Wrightstown & John Giberson & Greenhalgh \& Kaye & 1956 & 140 & 135 & 4 & -- & (none) & $\mathrm{Km}$ & 42 & 10 & 13 & $\mathrm{D}$ & & 135 \\
\hline 236 & Riverside & B. F. Goodrich Co. No. 2 & Artesian Well Drilling co. & 1951 & 34 & 162 & 10 & 95 & $95-120$ & $\ln n$ & 24 & 458 & 19 & Ind & A., L. & 136 \\
\hline 137 & I mi. SW of Riverside, at Cambridge & Dredge Harbor Yacht Basin & Charles L. Mollitor, Inc. & 1947 & 10 & 80 & -- & 76 & (none) & Pzw & -- & 25 & -- & cI & & 137 \\
\hline 138 & Riverside & Riverside-Alloy Metal Co. & Ridpath \& Potter & 1938 & 18 & 800 & 6 & 120 & (none) & Paw & -- & - & -- & Ind & L. & 138 \\
\hline 139 & do. & Riverside Industrial Center & Charles I. Mollitor, Inc. & 1952 & 18 & 80 & 8 & 54 & $54-67$ & $\mathrm{~K} \pi \mathrm{r}$ & 12 & 250 & 28 & Ind & $\begin{array}{l}\text { Water temperature re- } \\
\text { ported as } L \rho \circ F(\gamma)\end{array}$ & 139 \\
\hline $140^{\circ}$ & $1 \mathrm{mi}$. NW of Browns Mills & Jane Tabone & do. & 1954 & 130 & 140 & 4 & 134 & $134-140$ & Tvt & 38 & 10 & 8 & CI & L. & 240 \\
\hline 142 & 2 mi. $s$ of Pemberton & A. F. Brunt & do. & 1955 & 83 & 210 & 4 & 204 & $2014-210$ & $\mathrm{Km}$ & 45 & 15 & - & $D$ & L. & 241 \\
\hline 142 & $1 \mathrm{mi} . \mathrm{S}$ of Penbarton & Clarence Adans & J. Henry Robbins & 1949 & 72 & 195 & 4 & 175 & (none) & $\mathrm{Km}$ & 25 & 6 & -- & $\mathrm{D}$ & Farm use also. & 142 \\
\hline 143 & Browns willes & Meilley & Ridoath \& Potter & $\begin{array}{l}\text { before } \\
1910\end{array}$ & $70-80$ & 430 & 6 & -- & -- & Ket & (flowing) & 48 & -- & - & $\begin{array}{l}\text { Purped at a rate of } \\
225 \mathrm{gpm} \text { also. L. }\end{array}$ & 143 \\
\hline $1 / 4$ & $2 \mathrm{mi}$. E of Broms : vills, on Ridge Rd. & Joseph 0' Ne:111 & A. C. Schultes and Sons & 1949 & 91 & 337 & 6 & 322 & 322-337 & $\mathrm{x}$ mor & 29 & 110 & 27 & $\mathrm{D}$ & L. & 244 \\
\hline 2,5 & $\begin{array}{l}2 \text { mi. SE of Broms Ifills, at Country } \\
\text { Lakes }\end{array}$ & Country Lakes, Inc. & Charles L. Mollitar, Inc. & 1959 & 82 & 345 & 4 & 329 & $329-345$ & $\mathrm{xm}$ & 23 & 80 & 10 & PS & L. & 145 \\
\hline 146 & 2 mi. SE of Pemberton & Harvey Cower & Edward Robbins, sr. & 1949 & 68 & 250 & 6 & 250 & (none) & $\mathrm{Krm}$ & 14 & 150 & 86 & $\operatorname{Irr}$ & & 246 \\
\hline 4.7 & I mi. E of Pemberton, on State Rte 530 & John W. Coodman & Harvey Beebe, Sr. & 2951 & 73 & 231 & 2 & 140 & (none) & $\mathrm{Km}$ & 25 & 30 & - & $\mathrm{D}$ & & 47 \\
\hline 149 & $4 \mathrm{mi} . \mathrm{w}$ of Wrightstom, at Jobstom & Hel1s Stock Farm & $\begin{array}{l}\text { Wm. Blaisdell \& Orcutt } \\
\text { Bros. } \\
\text { Charles L. Mollitar, Inc. }\end{array}$ & $\begin{array}{l}\text { before } \\
1893 \\
1960\end{array}$ & $\begin{array}{c}\text { approx. } \\
70 \\
74\end{array}$ & 715 & - & -- & - & $\mathrm{Kmr}$ & - & -- & -- & $\mathrm{v}$ & $\begin{array}{l}\text { Abandoned. Formarly } \\
\text { called Lorillard well. } \\
\text { L. }\end{array}$ & 148 \\
\hline 150 & $3 \mathrm{mi}$. SW of Wrightstorm, at Juliustown & Militan L. Havens; Sr. & $\begin{array}{l}\text { Charles I. Mollitar, Inc. } \\
\text { Gus Hauser }\end{array}$ & 1953 & 1125 & 137 & 4 & 117 & (none) & $\begin{array}{l}\mathrm{K}_{\mathrm{et}} \\
\mathrm{K}_{\mathrm{m}}\end{array}$ & 20 & 200 & $\begin{array}{l}4 \\
2\end{array}$ & $\begin{array}{r}\mathrm{CI} \\
\mathrm{D}\end{array}$ & $\begin{array}{l}\text { A., } \mathrm{L} \text {. } \\
\text { Water terperature is } \\
56^{\circ} \mathrm{F} \text {. }\end{array}$ & $\begin{array}{l}149 \\
150\end{array}$ \\
\hline 251 & $3 \mathrm{mi}$. SW of wrightstom & Hells Stock Farm & Ridpath \& Potter & 1928 & 96 & 988 & $12 \& 8$ & 757 & $757-800$ & $\mathrm{Km} x$ & - & 220 & 4 & 0 & $\begin{array}{l}\text { Water contains iron. } \\
\text { Formerly called Walker- } \\
\text { Gordon Dairy well. L. }\end{array}$ & 151 \\
\hline $\begin{array}{l}152 \\
153\end{array}$ & $\begin{array}{l}3 \mathrm{mi} \cdot \mathrm{N} \text { of Mount Holly } \\
2 \mathrm{mi} \cdot \mathrm{NW} \text { of Wrightstomm }\end{array}$ & $\begin{array}{l}\text { Ridge Stables } \\
\text { Frank J. Ondrushek, Sr. }\end{array}$ & $\begin{array}{l}\text { Charles L. Mollitor, Inc. } \\
\text { Greenhalgh \& Kaye }\end{array}$ & 1957 & 41 & 230 & 6 & 220 & $220-230$ & $\ln x$ & 45 & 100 & 65 & $\mathrm{D}$ & Farm use also. L. & 252 \\
\hline 154 & $\begin{array}{l}5 \text { mi. NE of Mount HoLly, on U.s. } \\
\text { Highway } 206\end{array}$ & Tallman Brothers, Inc. & J. Henry Robbins & $\begin{array}{l}1958 \\
1956\end{array}$ & $\begin{array}{r}182 \\
42\end{array}$ & $\begin{array}{l}256 \\
260\end{array}$ & $\begin{array}{c}4 \\
8 \& 6\end{array}$ & $\begin{array}{l}253 \\
240\end{array}$ & $\begin{array}{l}253-256 \\
240-260\end{array}$ & $\begin{array}{l}\text { Ket } \\
\text { Knrr }\end{array}$ & $\begin{array}{l}96 \\
60\end{array}$ & $\begin{array}{l}15 \\
40\end{array}$ & $\begin{array}{l}30 \\
-\end{array}$ & \begin{tabular}{r|r}
$\mathrm{D}$ \\
$\mathrm{CI}$
\end{tabular} & & $\begin{array}{l}153 \\
154\end{array}$ \\
\hline 155 & do. & do. & W. R. Cladr & 1952 & 42 & 52 & 2 & 47 & 47-52 & Ket & 6 & 10 & 4 & & z. & 55 \\
\hline
\end{tabular}


Table 2.---Records of selected wells in Burlington County, N. J.--Contimued

\begin{tabular}{|c|c|c|c|c|c|c|c|c|c|c|c|c|c|c|c|c|}
\hline Well & Location & Owner or name and number & Driller & 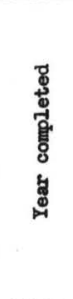 & 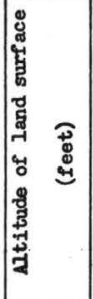 & 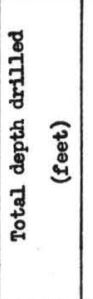 & 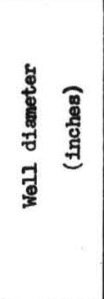 & 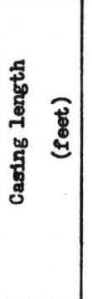 & 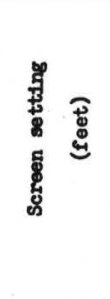 & 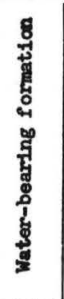 & 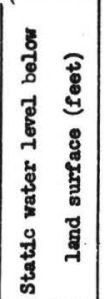 & 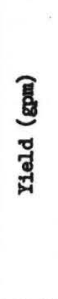 & 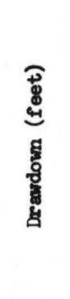 & $\stackrel{\Phi}{\square}$ & Remarkes & well \\
\hline 156 & $5 \mathrm{mi}$. SE of Mount Holly, at Vincentomm & Calvin G. Smith & Harvery Beebe, Sr. & 1949 & 37 & 271 & 2 & 17 & (nome) & $\mathbf{r}$ & 7 & 35 & 9 & D & & 256 \\
\hline 157 & $\begin{array}{l}6 \mathrm{mi} \text {. SE of Mount Holly, on } \mathrm{U}, \mathrm{S} \text {. } \\
\text { Hhry. } 206\end{array}$ & Ross Monroe & do. & 1949 & 46 & 248 & 2 & 157 & (none) & Kmmer & 211 & 25 & - & D & L. & 157 \\
\hline 158 & $\begin{array}{l}5 \text { mi. NE of Medford Lakes, at } \\
\text { Hampton Lakes }\end{array}$ & Hampton Lake Development Co. & Charles L. Mollitor, Inc. & 1956 & 49 & 268 & 10 & 247 & $247-268$ & $\mathbf{x} \operatorname{man}$ & 6 & 300 & $44^{2}$ & PS & A., $\mathrm{L}$. & 158 \\
\hline 159 & $\begin{array}{l}3 \text { mi. W of Pemberton, on HHghways } \\
38 \text { and } 206\end{array}$ & Vincentown Diner & Edward Robbins, Sr. & 1953 & 72 & 250 & 4 & 240 & (none) & Ket & 45 & 100 & 15 & CI & L. & 159 \\
\hline 160 & $\begin{array}{l}5 \text { mi. Ww of Chatsworth, on State } \\
\text { Highway } 72\end{array}$ & Frank Mayo & Greenhalgh \& Kaye & 1953 & 140 & 69 & 4 & 65 & $65-69$ & Toh & 37 & 25 & 9 & CI & L. & 160 \\
\hline 261 & $3 \mathrm{mi} . \mathrm{N}$ of Mount Holly & Ronald Smith & J. H. Jenkins and Sons & 1960 & 78 & 208 & 4 & 202 & 202-208 & $\mathrm{Kmr}$ & 90 & 25 & 60 & D & & 161 \\
\hline 162 & 2 mi. N af Mount Holly, on Oxmead Rd. & A. Marone & Charles L. Mollitor, Inc. & 1956 & 40 & 265 & 6 & 254 & $254-265$ & $\mathrm{Kmr}$ & 40 & 150 & 5 & D & $\begin{array}{l}\text { Supplies water to two } \\
\text { houses. L. }\end{array}$ & 162 \\
\hline 263 & $3 \mathrm{mi}$. $\mathrm{N}$ of Mount Holly & Clifford Seniner & do. & 1955 & 104 & 248 & 6 & 238 & $238-248$ & $\mathrm{Kmr}$ & 96 & 30 & 14 & D & & 163 \\
\hline 164 & 3 mi. W af Mount Holly & Walter Jessup & do. & 1950 & 80 & 241 & 4 & 234 & $234-242$ & $\mathrm{Kmr}$ & 90 & 4 & 50 & D & Water temp. is $54^{\circ} \mathrm{T}$. & 164 \\
\hline 165 & do. & Alexander Construction co. & do. & 1955 & 57 & 215 & 6 & 204 & $204-2214$ & $\mathrm{Kmr}$ & 60 & 50 & 20 & $\mathbf{v}$ & Abandoned. L. & 165 \\
\hline 166 & $\begin{array}{l}3 \mathrm{mi} \text {. WW of Mount Holly, an Woodlane } \\
\text { Rd. }\end{array}$ & John S. Pew & do. & 1957 & 79 & 228 & 4 & 222 & $222-228$ & $\mathrm{Kmr}$ & 60 & 20 & 10 & D & L. & 266 \\
\hline 167 & 3 mi. $S$ of Burlington & Eugene D. Buzzeli & do. & 1956 & 45 & 211 & 4 & 204 & 204-210 & $\mathbf{x m}$ & 48 & 20 & 6 & D & & 167 \\
\hline 168 & $2 \mathrm{md}$. $\mathrm{N}$ of Mount Holly, on Burr Rd. & Burlington Co. Country Club & Edward Robbins, Sr. & 1950 & 102 & 309 & $8 \& 6$ & 269 & 269-309 & $\mathbf{x}$ & 100 & 400 & 20 & Irx & $\begin{array}{l}\text { Supplies water to the } \\
\text { clubhouse also. } \mathrm{A} \text {. }\end{array}$ & 168 \\
\hline 169 & I ml. E of Mount Holly, on Powell Rd. & John W. Hampton & Charles L. Mollitor, Inc. & 1955 & 55 & 160 & 4 & 154 & $154-160$ & Ket & 27 & 45 & 28 & $\mathrm{D}$ & L. & 169 \\
\hline 170 & $2 \mathrm{md}$. E of Mount Holly, on Powell Rd. & Donald C. Foote & do. & 1954 & 52 & 160 & 4 & 254 & $154-160$ & Ket & 25 & 25 & 7 & D & & 170 \\
\hline 17 & 2 mil. W of Pamberton, at Bimingham & Ionac Chemical co. & A. C. Schultes \& Sons & 1960 & 30 & 921 & 12 & 490 & $490-521$ & Kim & 39 & 1001 & 104 & Ind & A., L. & 17 \\
\hline 172 & $\begin{array}{l}2 \mathrm{mil} \text {. B of Pemberton, on State Route } \\
530\end{array}$ & Burlington Co. Institution No.I & Layne-New York Co., Inc. & 1931 & 59 & 776 . & $12 \& 3$ & 353 & $353-381$ & Ket & 42 & - & - & CI & A., L. & 172 \\
\hline 173 & 4 mi. $N$ af Modford & Lester Jones & Charles L. Mollitor, Inc. & 1953 & 42 & 159 & 6 & 148 & $148-159$ & Ket & 16 & 210 & 34 & D & Farm use also. A. & 173 \\
\hline 174 & Mount Holly & Mount Holly Water Co. No. 1 & Layne-New Yark Co., Inc. & 1925 & 9 & 376 & $18 \& 10$ & 322 & $322-372$ & $\mathrm{Km}$ & 12 & 700 & 101 & PS & A. & 174 \\
\hline 175 & $1 \mathrm{ml} . \mathrm{SW}$ of Florence & National Cypsum Co. No. 1 & do. & 1956 & 22 & 167 & $12 \& 8$ & 228 & $118-128$ & $\mathrm{Kmr}$ & 19 & 154 & 38 & Ind & A. & 175 \\
\hline 176 & $\begin{array}{l}7 \text { mi. N of Wrightstove, on Emlisdale } \\
\text { Bd. }\end{array}$ & R. H. Clark & J. Henry Robbins & 1952 & 87 & 95 & 4 & 85 & $85-95$ & Ket & 40 & 20 & - & D & A. & 176 \\
\hline 177 & 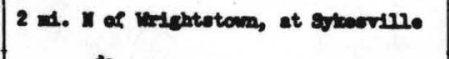 & Hen Horbert & Groenhaleh \& Kasp & 2956 & 170 & 29 & 4 & 225 & (nome) & $\mathrm{r}=$ & 40 & 10 & 20 & D & A. & $1 \pi$ \\
\hline 178 & - & Willim C. Rahills & do. & 1955 & 195 & 258 & 4 & 255 & $255-158$ & $x=$ & 58 & 10 & 5 & Df & I. & 170 \\
\hline 27 & $\begin{array}{l}2 \text { ac. I of mightotom, on State Route } \\
528 \mathrm{spur}\end{array}$ & Dix Conereto Co. & Leon Gager & 1953 & 100 & 117 & 6 & 100 & $100-110$ & $\mathrm{Km}$ & (flowing) & 60 & 68 & CI & 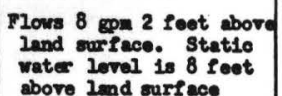 & 179 \\
\hline 280 & No-1entstoven & Clifeard C. Holden & Greenhalgh \& Kaye & 1957 & 233 & 150 & 4 & -- & (none) & $\mathbf{x}=$ & 20 & 15 & 20 & D & 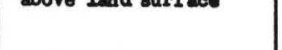 & 180 \\
\hline 181 & do. & $\begin{array}{l}\text { Wrightstore Municipal Vtility } \\
\text { Authority }\end{array}$ & & --- & 120 & 268 & 8 & -- & - & Ket & (ellowing) & -- & - & 0 & A., l & 201 \\
\hline
\end{tabular}




\begin{tabular}{|c|c|c|c|c|c|c|c|c|c|c|c|c|c|c|c|c|}
\hline Well & Location & Omer or name and number & Driller & 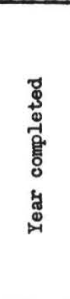 & 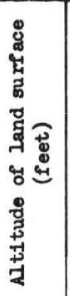 & 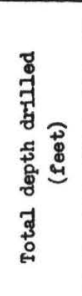 & 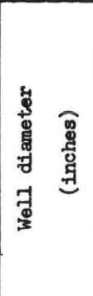 & 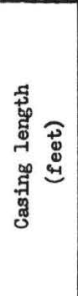 & 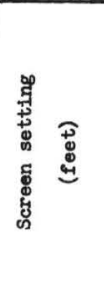 & 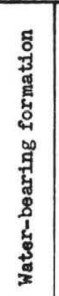 & 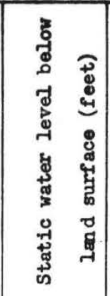 & 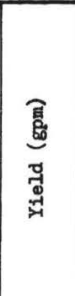 & 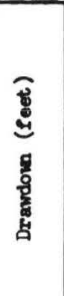 & $:$ & Remarks & noll \\
\hline 182 & $1 \mathrm{mi}$. W of Wrightstown & John I. Bond & Greenhalgh \& Kaye & 1953 & 143 & 123 & - & - & --- & $\mathrm{Kmm}$ & 13 & 15 & 7 & D & & 182 \\
\hline 183 & $2 \mathrm{mi} . \mathrm{W}$ of Wrightstowm & Charles B. Hutchinson & Charles L. Mollitor, Inc. & 1960 & 180 & 134 & 4 & 124 & $124-134$ & Kmw & 54 & 25 & 5 & $\mathrm{D}$ & L. & 183 \\
\hline 184 & $2 \mathrm{mi} . \mathrm{NE}$ of Browns Mills & Fort Dix No. 10 & Willilam Stothoff co., Inc. & 1943 & 100 & 449 & 6 & -- & ---- & Ket & 26 & 60 & 102 & PS & a. & 184 \\
\hline 185 & $4 \mathrm{mi}$. SE of Broums wels, at Whitesbog & Joseph J. White, Inc. & Ridpath \& Potter & 1914 & 95 & 801 & $10 \& 8$ & 498 & (none) & Ket & 5 & 45 & - & u & L. & 185 \\
\hline 186 & do. & do. & J. W. Jenkins & 1959 & 95 & 388 & $6 \& 4$ & 376 & $376-388$ & $\mathrm{Kmm}$ & - & 150 & -- & CI & $\begin{array}{l}\text { Pumping level is } 90 \text { feet. } \\
\text { L. }\end{array}$ & 186 \\
\hline 187 & $4 \mathrm{mi}$. SE of Pemberton & Francis Bush & Edward Robbins, Sr. & 1954 & 65 & 273 & 6 & 250 & $250-256$ & $\mathrm{Kmw}$ & 28 & 400 & 38 & $\operatorname{Irx}$ & & 187 \\
\hline 188 & Chatsworth & Anthony DeMarco Cranberry Plant & Bernard A. Leek & -- & 99 & 67 & 3 & - & --- & $\operatorname{Tch}$ & 6 & - & - & D & A.: & 188 \\
\hline 189 & $\begin{array}{l}3 \text { mi. NE of New Gratna, at Lake } \\
\text { Absegami }\end{array}$ & Bass River State Forest & & ---- & 20 & 31 & $1 \frac{1}{4}$ & 27 & (none) & Teh & 3 & - & - & PS & A. & 189 \\
\hline 190 & $6 \mathrm{mi} . \mathrm{W}$ of New Gretna & Green Bank State Forest Nursery & & & 13 & 73 & $8 \& 6$ & 53 & $53-73$ & $\mathrm{Tch}$ & - & 150 & -- & $\operatorname{Irr}$ & A. & 190 \\
\hline 191 & New Gretna & Chester Allen & & & 9 & 232 & 3 & -- & --.-- & Thw & (rlowing) & 20 & 4 & CI & $\begin{array}{l}\text { Static water level is } 4 \\
\text { feet above land surface. } \\
\text { A. }\end{array}$ & 191 \\
\hline 192 & Atsion & Lesson Small & & & 51 & 88 & $1 \frac{1}{4}$ & -- & $-\cdots--$ & $\operatorname{Tch}$ & 6 & - & - & D & A. & 192 \\
\hline 193 & $3 \mathrm{mi} . \mathrm{N}$ of Atsion, at Indian Mills & Leah Prickett & & --- & 77 & 60 & - & -- & ----- & Teh & - & -- & - & D & A. & 193 \\
\hline 194 & $4 \mathrm{mi}$. SW of Pemberton, near Vincentown & Vincentown water Co. & & 1923 & 40 & 153 & 8 & -- & --.-- & Knar & (rlowing) & 175 & - & PS & $\begin{array}{l}\text { Flows } 28 \mathrm{gpm} \text { at land sur- } \\
\text { face in the spring seasor } \\
\text { A. }\end{array}$ & 194 \\
\hline 195 & $2 \mathrm{mi} . \mathrm{S}$ of Mount Holly, at Lumberton & Electronic Parts Specialty Co. & J. Henry Robbins & 1953 & 33 & 121 & $6 \& 4$ & 101 & $101-121$ & Ket & 30 & 50 & -- & Ind & A. & 195 \\
\hline 196 & $\begin{array}{l}2 \text { mi. } W \text { of Medford Lakes, at Taunton } \\
\text { Lakes }\end{array}$ & Taunton Lakes Water Co. No. 1 & do. & 1950 & 57 & 252 & 6 & 230 & (none) & Kmar & 25 & 300 & - & $\mathrm{v}$ & Abandoned. A., L. & 196 \\
\hline 197 & Beverly & National Wat $\in$ roroof Papers, Inc. & Charles L. Mollitor, Inc. & 1955 & 38 & 61 & 16 & 41 & $41-6$ & $\mathrm{Kmr}$ & 22 & -- & - & Ind & Used for cooling. & 197 \\
\hline 198 & 2 mi. SE of Riverton & $\begin{array}{l}\text { Ríverton-Palnura Water Co. } \\
\text { No. } 10\end{array}$ & A. C. Schultes \& Sons & 1961 & 79 & 308 & 12 & 243 & $243-281$ & $\operatorname{Knr}$ & 75 & 1051 & 95 & PS & $\begin{array}{l}\text { Drilled to weathered bed- } \\
\text { rock. A., L. }\end{array}$ & 198 \\
\hline 199 & $2 \mathrm{mi} \mathrm{NE}$ of Moorestown, on Hartford $\mathrm{Rd}$. & Moorestown Tp. Water Dept. No. 4 & Layne-New York Co., Inc. & 1959 & 59 & 360 & 12 & 298 & $298-338$ & $\mathbf{K m r}$ & 58 & 715 & 52 & Ps & L. & 199 \\
\hline 200 & $2 \mathrm{mi} \mathrm{NE}$ of Chatsworth & The Superior Zinc Corp. & Artesian well Drilling Co. & 1941 & 140 & 153 & 12 & 121 & $121-1146$ & Teh & 36 & 300 & 50 & Ind & & 200 \\
\hline 201 & $6 \mathrm{mi} . \mathrm{E}$ of Chatoworth on State Hiry 72 & Cedar Bridge Fire Tower & J. Henry Robbins & 1938 & 200 & 2110 & 6 & -- & $-\cdots$ & Tch & 61 & -- & - & 0 & I. & 201 \\
\hline 202 & $3 \mathrm{mi}$. W of Mount Holly & Inductotherm corp. & Charles L. Mollitor, Inc. & 1961 & 65 & 444 & 6 & 471 & $472-443$ & $\mathrm{Kmr}$ & 80 & 300 & 16 & Ind & I. & 202 \\
\hline 203 & $\begin{array}{l}6 \text { mi. S af Chatsworth, on State } \\
\text { Route } 563\end{array}$ & Arthur Sooy & (omer) & -- & 45 & 30 & 2 & -- & ----- & Teh & - & -- & -- & $\mathrm{D}$ & Driven well. A. & 203 \\
\hline 204 & I mi. SW of Bordentom at Fleldsboro & Bzura Chemical Co., Inc. & A. J. Connelly, Inc. & 1960 & 9 & 79 & - & -- & --- & $\mathrm{Kmr}$ & - & -- & - & Ind & A. & 204 \\
\hline 205 & $3 \mathrm{mi} . \mathrm{NE}$ of Pemberton & Pemberton Tp. High School & Legno-Hew Yark Co., Ine. & 1959 & 75 & 220 & 6 & 140 & $140-150$ & $x=$ & - & -- & -- & CI & A. & 205 \\
\hline 206 & $3 \mathrm{mi} . \mathrm{E}$ of Moorestom on Marne thy & William Haines, Jr. & Artesian well Drilling co. & 1958 & 55 & 238 & 8 & 200 & $200-238$ & Set & - & 400 & - & Irr & a. & 206 \\
\hline $\begin{array}{l}207 \\
208\end{array}$ & $\begin{array}{l}5 \mathrm{mi} \text {. AE af Mount Holly on U.S. Hhy } 206 \\
2 \mathrm{mi} \text {. S of Moorestown }\end{array}$ & $\begin{array}{l}\text { Harry Rupp } \\
\text { Mount Laurel Water co. }\end{array}$ & A. C. Schultes \& Sons & --- & $\begin{array}{l}62 \\
30\end{array}$ & $\begin{array}{r}52 \\
595\end{array}$ & $\begin{array}{c}4 \\
12,8\end{array}$ & -- & 558-589 & $\begin{array}{l}\text { Iet } \\
\operatorname{Imr}\end{array}$ & - & 548 & - & $\begin{array}{l}\text { CI } \\
\text { PS }\end{array}$ & A. & $\begin{array}{l}207 \\
208\end{array}$ \\
\hline
\end{tabular}


Table 3. - Selected drillers' logs of wells in Burlington County, N. J.

Well no. 3, Burlington Water Department

(Log by Layne-New York Co., Inc.)

Topsoil . ........................

Fill

Quaternary:

Cape May Formation:

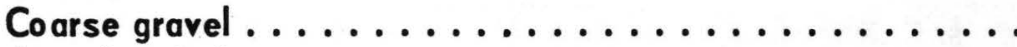

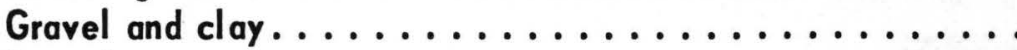

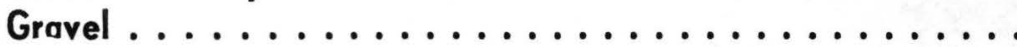

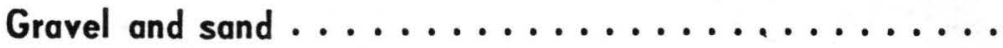

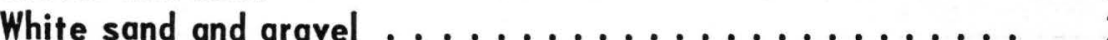

Brown sand and clay $\ldots \ldots \ldots \ldots \ldots \ldots \ldots, 7$

Altitude 9 feet

Thickness

(feet)

1

4

Depth

(feet)

Craceous:

Magothy (?) and Raritan (?) Formations:

White sand and clay.

10

43

Sand, gravel, and sandy clay.

8

51

Magothy and Raritan Formations:

Blue clay ...................... 10

Clay, gravel, and sand $\ldots \ldots \ldots \ldots \ldots \ldots \ldots \ldots$

Gravel, sand and clay $\ldots \ldots \ldots \ldots \ldots \ldots \ldots \ldots$

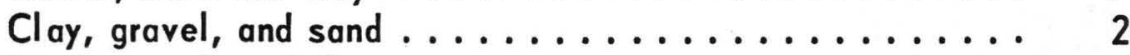

Sand, gravel, and clay ................ 12

Quartz sand and blue clay............... 5

590


Table 3. - Selected drillers' logs of wells in Burlington County, N. J. - Continued

Well no. 4, Burlington Water Department

(Log by Artesian Well Drilling Co.)

\begin{tabular}{|c|c|c|}
\hline & \multicolumn{2}{|c|}{ Al titude 19 feet } \\
\hline & $\begin{array}{l}\text { Thickness } \\
\text { (feet) }\end{array}$ & $\begin{array}{l}\text { Depth } \\
\text { (feet) }\end{array}$ \\
\hline Sand and loam ... & 5 & 5 \\
\hline Dirty, brown sand & 5 & 10 \\
\hline Coarse sand $\ldots \ldots \ldots \ldots \ldots \ldots \ldots \ldots$ & 5 & 15 \\
\hline Coarse sand and mud, some gravel $\ldots \ldots \ldots \ldots \ldots \ldots$ & 5 & 20 \\
\hline Coarse sand and medium gravel $\ldots \ldots \ldots \ldots \ldots$ & 5 & 25 \\
\hline Coarse sand and gravel $\ldots \ldots \ldots \ldots \ldots \ldots \ldots \ldots \ldots$ & 5 & 30 \\
\hline Coarse sand and fine gravel $\ldots \ldots \ldots \ldots \ldots \ldots \ldots$ & 10 & 40 \\
\hline Coarse sand and gravel $\ldots \ldots \ldots \ldots \ldots$ & 6 & 46 \\
\hline Medium and large gravel $\ldots .$. & 2 & 48 \\
\hline $\begin{array}{l}\text { Cretaceous: } \\
\text { Magothy and Raritan Formations: }\end{array}$ & & \\
\hline Gray sandy clay $\ldots \ldots \ldots \ldots \ldots$ & 1 & 49 \\
\hline
\end{tabular}


Table 3. - Selected drillers' logs of wells in Burlington County, N. J. - Continued

Well no. 9, Delaware River Water Co.

(Log by A. C. Schultes \& Sons)

Quaternary:

Altitude 21 feet

Thickness Depth

(feet) (feet)

Cape May Formation:

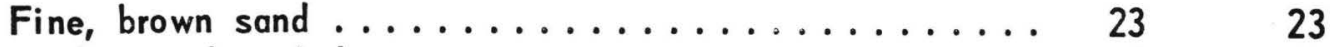

Sand, gravel, and clay .................. 326

Cretaceous(?):

Magothy (?) and Raritan (?) Formations:

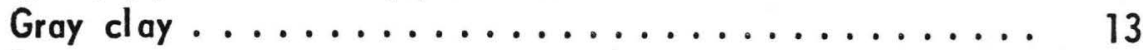

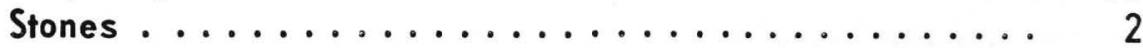

Interbedded brown sand, gravel, and clay .......... 8

Coarse, brown sand .................. 5

Cretaceous:

Magothy and Raritan Formations:

Coarse, brown sand and white cloy, some gravel ....... $21 \quad 75$

Gravel and yellow clay .................. 7 . 82

Red, white and yellow clay ................. 89

Red and brown clay .................... 6 . . 96

Yellow and white clay .................. 2 2

Stones, gravel, and clay.................. 7 105

Fine sand and gravel ..................... 4 . 109

Fine sand and gravel, some white clay............ $10 \quad 119$

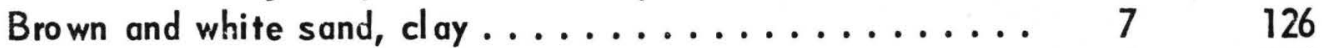

Coarse, brown sand and clay ................ 64132

Yellow and white clay .................. 32135

Early Paleozoic (?):

Wissahickon Formation:

Weathered rock .................... 
Table 3. - Selected drillers' logs of wells in Burlington County, N. J. - Continued

Well no. 10, Delaware River Water Co.

(Log by A. C. Schultes and Sons)

Cuaternary:

Altitude 25 feet
Thickness Depth
(feet) (feet)

Cape May Formation:

No sample ................................. $20 \quad 20$

Cretaceous (?):

Magothy (?) and Raritan (?) Formations:

Hard clay ................................... 15

Cretaceous:

Magothy and Raritan Formations:

White and red clay ...............................

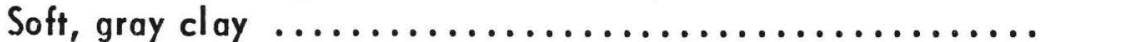

Yellow sandy clay ............................. 14

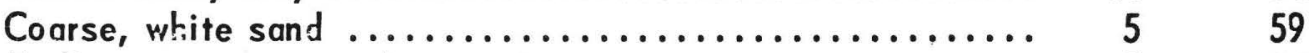

Medium to coarse sand, some ironstone ............... 5

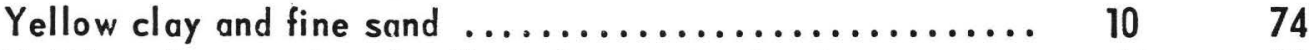

Pebbles, fine sand, and yellow clay - water-bearing ........ 7 . 71

Medium to coarse sandy clay, ironstone ................ 5

Coarse sand and gravel ........................... 5

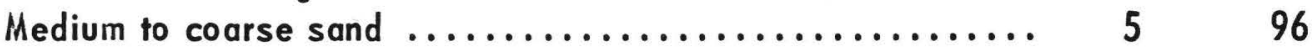

Medium to coarse sand, clay, and stones............... $18 \quad 114$

Fine sand, stones, and yellow clay .................. $5 \quad 119$

Medium to coarse sand ............................ $10 \quad 129$

Brown clay ................................... 7

Red clay ................................. $6 \quad 142$

Early Paleozoic (?):

Wissahickon Formation:

Weathered rock ................................ I 143 
Table 3. - Selected drillers' logs of wells in Burlington County, N. J. - Continued Well no. 11, Evesham Township Water Department

(Log by A. G. Dunphey)

Topsoil

Quaternary:

Pensauken Formation:

Yellow gravel ................... 5

Altitude 115 feet

Thickness Depth

(feet) (feet)

$9 \quad 9$

Tertiary:

Kirkwood Formation:

Yellow sand....................... 15

Tertiary and Cretaceous:

Hornerstown Sand and Navesink Formation (undifferentiated):

Black mud....................... 20

Marl .......................... 25

Brown mud....................... 19

Mud and shells................... 3

Cretaceous:

Mount Laurel Sand and Wenonah Formation:

Gray sand with abundance of water, somewhat irony ..... 10

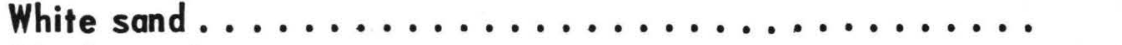

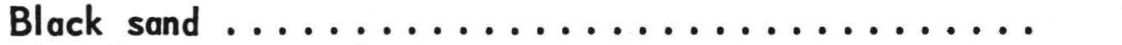

Whitish or gray sand $\ldots \ldots \ldots \ldots \ldots \ldots \ldots \ldots \ldots$

Black sandy mud

At

29

Marshalltown (?) Formation:

Black clay ............................. Below

Englishtown (?) Formation:

Black, muddy sandy................ At

Englishtown Formation:

Sandy marl

.........

Light-colored sand.

At

196

208

Water-bearing, yellowish-white sand.

At

212 
Table 3. - Selected drillers' logs of wells in Burlington County, N. J. - Continued

Well no. 12, Evesham Township Water Department

(Log by Layne-New York Co., Inc.)

Altitude 89 feet

Thickness Depth

(feet) (feet)

Topsoil ...................... 1 1

Tertiary:

Hornestown Sand:

Yellow sandy clay, red and blue marl ............ $15 \quad 16$

Cretaceous:

Navesink Formation:

Black zeolite (?) sand and marl with clay streaks ...... $57 \quad 73$

Mount Laurel Sand, Wenonah Formation, and Marshall town

Formation (undifferentiated):

Fine to coarse sand with shells . . . . . . . . . . . $37 \quad 110$

Blue clay with sand streaks............... $55 \quad 165$

Englishtown Formation:

Fine to medium sand and gravel ............ $35 \quad 200$

Woodbury $\mathrm{Cl}$ ay and Merchantville Formation (undifferentiated):

Blue clay and sand with shells .............. $76 \quad 276$

Blue and white clay with sand streaks............ $84 \quad 360$

Magothy and Raritan Formation s:

Fine to coarse, black and white sand .......... 33

Blue clay with streaks of fine sand . . . . . . . . . . $22 \quad 415$

Fine to medium sand .................. $11 \quad 426$

Fine to medium sand with blue and white clay streaks. . . . $74 \quad 500$

Blue, red, and white clay ................. $12 \quad 512$ 
Table 3. - Selected drillers' logs of wells in Burlington County, N. J. - Continued

Well no. 13, Florence Township Water Department

(Log by Layne-New York Co., Inc.)

Altitude 25 feet

Thickness Depth

(feet) (feet)

Quaternary:

Cape May Formation:

Fine, brown sand $\ldots \ldots \ldots \ldots \ldots \ldots \ldots \ldots \ldots \ldots$

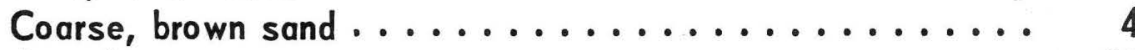

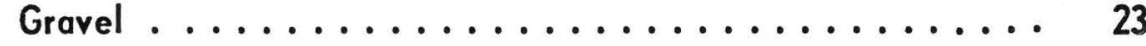

4

$23 \quad 31$

Cretaceous:

Magothy and Raritan Formations:

Blue and white clay ..................... 2 33

Tough red clay....................... 7 74

Tough red and white clay ................... 44

Blue and white sandy clay ................... 10

Medium gray sand and white clay ............... 19

Coarse sand, red and white clay .............. $12 \quad 125$

Coarse, brown sand .................... $13 \quad 138$

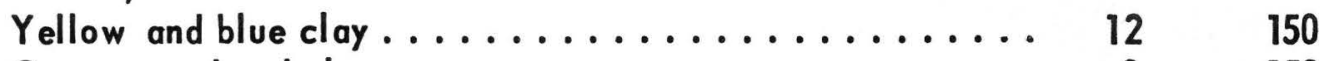

Coarse sand and clay ................... 8

Early Paleozoic (?):

Wissahickon Formation:

Mica rock ........................ 5 
Table 3. - Selected drillers' logs of wells in Burlington County, N. J. - Continued

Well no. 15, Fort Dix

(Log by Artesian Well Drilling Co.)

Tertiary:

$\begin{array}{cc}\text { Altitude } 131 \text { feet } \\ \text { Thickness } & \text { Depth } \\ \text { (feet) } & \text { (feet) }\end{array}$

Kirkwood Formation:

Fine, yellow clayey sand.................... 22

Gray clay and fine silty sand ................. 18 40

Green, clayey, glauconitic sand .............. $25 \quad 65$

Manasquan and Vincentown Formations, Hornerstown Sand

(undifferentiated):

Yellowish-green, clayey, glauconitic sand .......... $41 \quad 106$

$\begin{array}{llll}\text { Bright-green, clayey, and sandy glauconite with shells... } & 14 & 120\end{array}$

Cretaceous:

Navesink Formation:

Dark-gray glauconitic clay with shells........... $21 \quad 141$

Medium, yellowish-green, glauconitic sand .......... 21

Mount Laurel Sand and Wenonah Formation:

Gray, clayey, glauconitic sand with fossils.......... At

Fine, gray, clayey micaceous sand ............. At

162

Marshalltown Formation:

Gray clay and fine, micaceous sand ............. At 274

Dark greenish-gray glauconitic clay with some fine

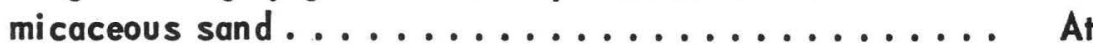

275

Englishtown Formation:

Brown clay with a trace of lignite . . . . . . . . . . . At 299

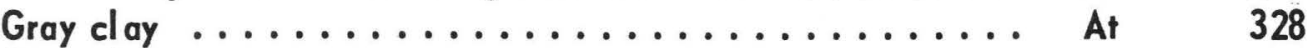

Gray clay ........................... At 352

Woodbury Formation:

Gray, glauconitic, fossiliferous clay ............ At 370

Gray, glauconitic, fossiliferous clay ............. $77 \quad 447$

Merchantville Formation:

Greenish-gray to dark gray glauconitic clay ......... $83 \quad 530$

Magothy and Raritan Formations:

Fine to medium glauconitic sand ............ At

At $\quad 530$

Very fine sand ....................... At 540

Very fine sand and some clay ............... $40 \quad 580$

Fine sand ............................ $42 \quad 622$

Fine to coarse sand and gravel ................. 6628

Fine to coarse, gray slightly clayey sand .......... 2 630

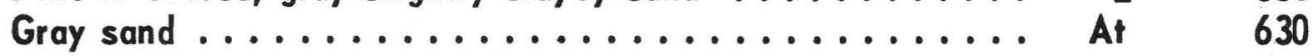

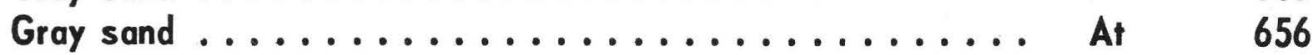

Very fine, light gray silty sand $\ldots \ldots \ldots \ldots \ldots$.......... At 663

Fine to coarse, light gray sand, gravel and white clay .... At 668

Fine, brown, micaceous sand ............... At 715

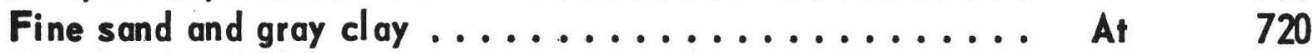

Fine sand and clay ..................... $150 \quad 870$

Red, white, and gray clay ................... $160 \quad 1030$

Coarse water-bearing sand $\ldots \ldots \ldots \ldots \ldots \ldots$........... 21 
Table 3. - Selected drillers' logs of wells in Burlington County, N. J. - Continued

Well no. 16, Fort Dix

(Log by Layne-New York Co., Inc.)

Tertiary:

Altitude 148 feet
Thickness $\begin{aligned} & \text { Depth } \\ & \text { (feet) }\end{aligned}$

Undifferentiated:

Sand and gravel ................. 82

Clay and black sand ............... 59

Cretaceous:

Undifferentiated:

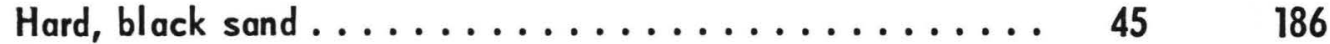

Silt and clay....................... 45

Gray sand and gravel (?) ................ $25 \quad 256$

Marshall town and Englishtown Formations (undifferentiated):

Silt, sand, and clay ................... $42 \quad 298$

Woodbury Clay and Merchantville Formation (undifferentiated):

Black clay and sand .................. $68 \quad 366$

Clay and sand ..................... $56 \quad 422$

Hard clay and sand .................... 101

Magothy (?) and Raritan (?) Formations:

Sandy clay......................... $33 \quad 556$

Tough clay ......................... $12 \quad 568$

Sandy clay ....................... $68 \quad 636$

Hard sandy clay ..................... $58 \quad 694$

Tough clay with hard streaks. . . . . . . . . . . . $48 \quad 742$

Clay and gravel ..................... 7

Gravel and hard clay ..................... $23 \quad 772$

Sand and hard clay ........................ 50

Magothy and Raritan Formations:

Clay with red streaks . . . . . . . . . . . . . . . $87 \quad 909$

Clay, sand, gravel, and wood ............... $20 \quad 929$

Medium, gray sand . . . . . . . . . . . . . . . . . . . 19

Tough clay . . . . . . . . . . . . . . . . . . . . . 21 969

Muddy sand .................... $12 \quad 981$

Tough clay ......................... $22 \quad 1003$

Medium, gray sand.................. $21 \quad 1024$

Tough clay . . . . . . . . . . . . . . . . . . . . . . $10 \quad 1034$

Sand, clay, and gravel ................... 16 1050

Coarse, gray sand and gravel . . . . . . . . . . . . 36 1086

Clay.......................... 10 1096 
Table 3. - Selected drillers' logs of wells in Burlington County, N. J. - Continued Well no. 17, Fort Dix (Log by H. G. Richards, Geologist)

Tertiary:

Altitude 85 feet

Thickness Depth

(feet) (feet)

Kirkwood Formation:

Fine, yellow, slightly clayey sand . . . . . . . . . . . $10 \quad 10$

Fine to coarse, buff and light gray sand . . . . . . . . $10 \quad 20$

Fine sand and gray clay ................. $10 \quad 30$

Very fine, gray, clayey sand ................ $30 \quad 60$

Very fine, gray sand and clay ............... $10 \quad 70$

Black, lignitic, silty clay ................... $10 \quad 80$

Manasquan and Vincentown Formations (undifferentiated):

Green clay........................ $50 \quad 130$

Green glauconitic clay . . . . . . . . . . . . . . . 10 10

Hard, light green clay . . . . . . . . . . . . . . . 20 $20 \quad 160$

Green clay........................... 30 190

Green glauconitic clay ................... 10 10200

Green clay ........................ $10 \quad 210$

Hornerstown Sand:

Dark green to gray glauconitic clay . . . . . . . . . . $20 \quad 230$

Green glauconitic clay ................... $10 \quad 240$

Green glauconitic sandy clay . . . . . . . . . . . . . . 20 2026

Cretaceous:

Navesink Formation:

Gray glauconitic clay. . . . . . . . . . . ... $10 \quad 270$

Gray sandy clay .................... $30 \quad 300$

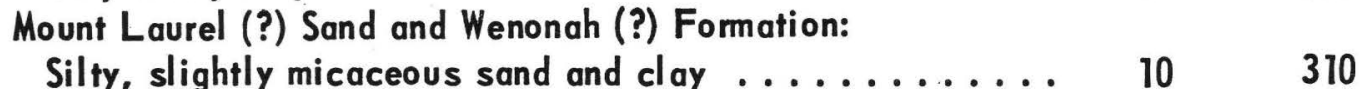

Mount Laurel Sand and Wenonah Formation:

Green silty, slightly clayey sand . . . . . . . . . . 30 30

Gray silty sand and clay ................. $50 \quad 390$

Marshal Itown Formation:

Gray, silty, sandy clay . . . . . . . . . . . . . . . . $50 \quad 440$

Fine, green to gray, clayey, glauconitic sand . . . . . . . $10 \quad 450$

Englishtown Formation:

Gray clay and sand .................. $10 \quad 460$

Fine to medium, light green to gray, slightly glauconitic sand with shell fragments............... 25 
Table 3. - Selected drillers' logs of wells in Burlington County, N. J. - Continued Well no. 18, McGuire Air Force Base

Topsoil (Log by A. C. Schultes and Sons)

Altitude 128 feet
Thickness
(feet)

Tertiary:

Kirkwood Formation:

Fine, yellow sand .................. 5

Fine, yellow sand and gravel . . . . . . . . . . . 9

Fine, gray sand .................. 7

Medium, yellow and gray sand .............. 13

Vincentown Formation:

Fine, gray sand .................... 15

Tertiary and Cretaceous:

Undifferentiated:

Gray and white sand, black marl with shells ........ 151

Blue clay and marl ................. 63

201

Cretaceous:

Englishtown Formation:

Streaks of hardpan and sand ............. 78

Gray sand with lignite, streaks of hardpan and shells . . . . $88 \quad 359$

Woodhury Clay:

Black clay with some sand ................ 16 375

Black clay, fine sand, and shells ............ $53 \quad 428$

Merchantville Formation:

Dark green clay, shells, and fine sand ........... 44432

Clay and medium sand .................. 13

Black clay, medium sand, and shells ............ $57 \quad 502$

Hiagothy (?) and Raritan (?) Formations:

Green clay, fine sand, and black clay . . . . . . . . $48 \quad 550$

Magothy and Raritan Formations:

Red and black clay with hard sand streaks . . . . . . . $38 \quad 588$

Gray sand and white clay ............... $53 \quad 641$

Gray sand with streaks of black clay . . . . . . . . . . . $24 \quad 665$

Black clay........................ $5 \quad 670$

Black clay and fine sand ................ 5

Fine, gray sand and black clay ............. 5

Fine to medium, gray sand and black clay ......... $41 \quad 721$

Black clay and very fine sand ................ $19 \quad 740$

Black clay, red clay, and shells................ 12

Red and black clay, shells, and fine sand .......... $23 \quad 775$

Fine, gray and white sand ............... $50 \quad 825$

Black and red clay, wood, and black and gray sand . . . . . 55

Gray sand, red clay, and wood . . . . . . . . . . . . . $39 \quad 919$

Tough, variegated clay................... 3195

Hard, red clay and coarse sand . . . . . . . . . . . . $5 \quad 955$

Hard, variegated clay .................. 596

Very hard, red clay ........................ $24 \quad 984$

Medium to coarse sand .................... $44 \quad 1028$

Fine to coarse sand ..................... $32 \quad 1060$

Early Paleozoic (?):

Wissahickon (?) Formation:

Clay, sand, and weathered rock ............. $37 \quad 1097$

Red clay ....................... $3 \quad 1100$ 
Table 3. - Selected drillers' logs of wells in Burlington County, N. J. - Continued

Well no. 19, McGuire Air Force Base

(Log by A. C. Schultes and Sons)

Tertiary:

$\begin{array}{cr}\text { Altitude } 102 \text { feet } \\ \text { Thickness } & \text { Depth } \\ \text { (feet) } & \text { (feet) }\end{array}$

Kirkwood Formation:

Yellow sand ...................... 5

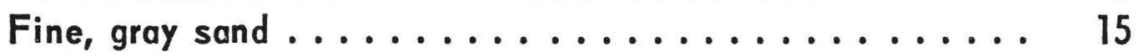

Yellow clay, sand, and wood ..........................

Hard, gray and white sand ................ 65

Cretaceous:

Navesink Formation:

Black marl with shells ..................... 40

Red clay ..........................

Mount Laurel Sand and Wenonah Formation:

Fine, black and white sand ............... 89

220

Marshall town Formation:

Fine, gray and black sand ............... 32

252

Gray clay

Englishtown Formation:

Fine to medium, gray, micaceous sand .......... 25

285

Medium to coarse sand, black marl, and shells ....... 20

305

Coarse sand, black marl, and shells ............ 25

330

Coarse sand with shells.

350

Woodbury Clay:

Fine to coarse sand (?), marl, and shells . . . . . . . . 25

Fine to medium sand (?), shells, and marl ......... 35

375

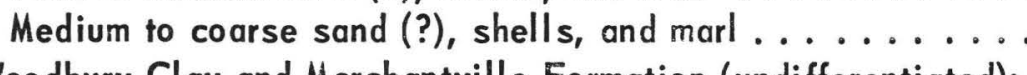

410

Woodbury Clay and Merchantville Formation (undifferentiated):

Medium to coarse sand (?), red clay, marl and shells . . . . 38

425

Merchantville Formation:

Marl, shells, and red clay ................ 67

463

Magothy and Raritan Formations:

Clay, shells, and wood..................

Clay, shells, white clay, and wood ..............

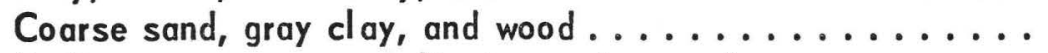

Medium to coarse sand, lignite, and gray clay .........

Red and white clay and marl .................

Layers of gravel, wood, and micaceous clay ..........

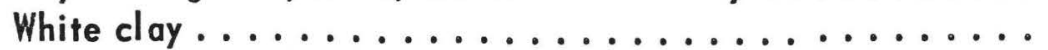

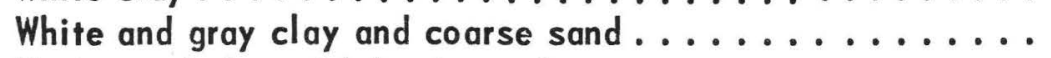

Variegated clay with hard streaks ..............

Variegated clay ...................... 70

Gray sand, streaks of red and white clay, and wood ...... 62

Medium to coarse sand and white clay ............ 6

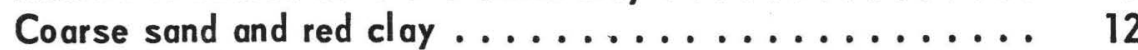

Sand and red clay .................... 20

Red clay .......................... 8

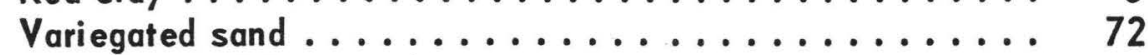


Table 3. - Selected drillers' logs of wells in Burlington County, N. J. - Continued

Well no. 21, Lakes Water Co.

(Log by J. Henry Robbins)

Tertiary:

Kirkwood Formation:

Yellow sand and clay.

30

30

Kirkwood and Vincentown Formations (undifferentiated):

Black clay......................... $70 \quad 100$

Tertiary and Cretaceous:

Hornerstown Sand and Navesink Formation (undifferentiated):

Green clay........................... $70 \quad 170$

Cretaceous:

Mount Laurel Sand and Wenonah Formation:

Fine, green sand .................. 10

Coarse, green and white sand $\ldots \ldots \ldots \ldots \ldots \ldots \ldots$

180

200 
Table 3. - Selected drillers' logs of wells in Burlington County, N. J. - Continued Well no. 22, Levitt and Sons Inc.

(Log by A. C. Schultes and Sons)

\begin{tabular}{|c|c|c|}
\hline & Altitude & feet \\
\hline & $\begin{array}{l}\text { Thickness } \\
\text { (feet) }\end{array}$ & $\begin{array}{l}\text { Depth } \\
\text { (feet) }\end{array}$ \\
\hline$\ldots \ldots \ldots \ldots \ldots \ldots \ldots \ldots$ & 5 & 5 \\
\hline $\begin{array}{l}\text { Uaternary: } \\
\text { Cape May Formation: }\end{array}$ & & \\
\hline Fine white sand with mica $\ldots \ldots \ldots \ldots \ldots \ldots$ & 5 & 10 \\
\hline Fine to medium, brown sand $\ldots \ldots \ldots \ldots \ldots \ldots \ldots$ & 15 & 25 \\
\hline Coarse sand and marl $\ldots \ldots \ldots \ldots \ldots \ldots \ldots \ldots \ldots$ & 7 & 32 \\
\hline 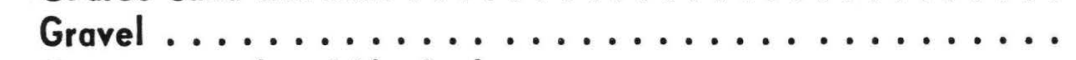 & 8 & 40 \\
\hline Coarse gravel and black clay $\ldots \ldots \ldots \ldots \ldots \ldots \ldots$ & 5 & 45 \\
\hline etaceous: & & \\
\hline Merchantville Formation: & & \\
\hline Black clay........... & 20 & 65 \\
\hline Magothy and Raritan Formations: & & \\
\hline Fine to medium, brown sand and clay $\ldots \ldots \ldots \ldots \ldots$ & 5 & 70 \\
\hline Fine, gray sand $\ldots \ldots \ldots \ldots \ldots \ldots \ldots \ldots \ldots$ & 15 & 85 \\
\hline Fine to medium gray sand with mica and wood $\ldots \ldots \ldots \ldots$ & 45 & 130 \\
\hline Medium to coarse sand $\ldots \ldots \ldots \ldots \ldots \ldots \ldots \ldots \ldots \ldots$ & 10 & 140 \\
\hline Fine to medium sand $\ldots \ldots \ldots \ldots \ldots \ldots \ldots \ldots \ldots \ldots$ & 35 & 175 \\
\hline White clay $\ldots \ldots \ldots \ldots \ldots \ldots \ldots \ldots \ldots$ & 19 & 194 \\
\hline Fine gray sand $\ldots \ldots \ldots \ldots \ldots \ldots \ldots \ldots \ldots \ldots \ldots$ & 51 & 245 \\
\hline edium, white sand $\ldots \ldots \ldots \ldots \ldots \ldots \ldots$ & 10 & 255 \\
\hline White clay and fine, white sand $\ldots \ldots \ldots \ldots \ldots \ldots \ldots$ & 10 & 265 \\
\hline$\ldots \ldots \ldots \ldots \ldots \ldots \ldots \ldots$ & 11 & 276 \\
\hline Gray clay, fine to coarse sand, and gravel $\ldots \ldots \ldots \ldots$ & 3 & 279 \\
\hline 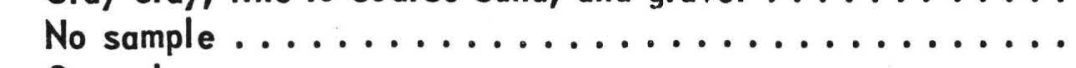 & 11 & 290 \\
\hline 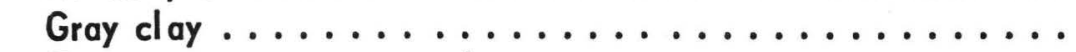 & 4 & 294 \\
\hline Fine to coarse, gray sand $\ldots \ldots \ldots \ldots \ldots \ldots \ldots \ldots \ldots$ & 29 & 323 \\
\hline Gray and red clay $\ldots \ldots \ldots \ldots \ldots \ldots \ldots \ldots$ & 15 & 338 \\
\hline Medium, gray sand $\ldots \ldots \ldots \ldots \ldots \ldots \ldots \ldots \ldots$ & 23 & 361 \\
\hline $\begin{array}{l}\text { arly Paleozoic (?): } \\
\text { Wissahickon Formation: }\end{array}$ & & \\
\hline & 2 & 363 \\
\hline
\end{tabular}


Table 3. - Selected drillers' logs of wells in Burlington County, N. J. - Continued Well no. 24, Levitt and Sons Inc.

(Log by A. C. Schultes and Sons)

Topsoil

Quaternary:

Cape May Formation:

Yellow sandy clay .................. 10

Altitude 28 feet

Thickness Depth

(feet) (feet)

Cretaceous:

Magothy and Raritan Formations:

Red and white clay .................. 39

Sand and gravel .................. 5

Dark gray clay ................... 5

$5 \quad 5$

Red clay with gravel .................. 16

Dark gray clay ......................

Medium, gray sand with clay .............. 11

Fine, brown sand .................. 15

Gravel with some stones................ 5

Medium, gray sand and gravel ............. 45

Medium to coarse sand and gravel ............ 40

Medium to coarse, white sand ............ 30

Gravel and red clay .................... 10

Gray silty clay .................... 3

Red clay ...................... 4

White silty clay $\ldots \ldots \ldots \ldots \ldots \ldots \ldots \ldots \ldots \ldots$

Fine to medium sand with clay ............. 18

Fine, brown sand with clay .............. 9

Hardpan ....................... I

arly Paleozoic (?):

Wissahickon Formation:

Weathered rock

80

84

95

110

115

160

200

230

240

243

247

255

273

282

283 
Table 3. - Selected drillers' logs of wells in Burlington County, N. J. - Continued

Well no. 25, Levitt and Sons Inc.

(Log by A. C. Schultes and Sons)

Fill

$\begin{array}{cc}\text { Altitude } 39 \text { feet } \\ \text { Thickness } \\ \text { Depth } \\ \text { (feet) } & \begin{array}{c}\text { (feet) } \\ \text {. } \\ 5\end{array} \\ \end{array}$

Cretaceous:

Magothy and Raritan Formations:

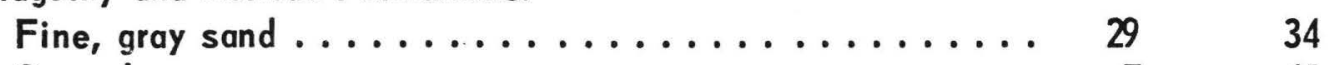

Gray clay ...................... 7

Fine, gray sand $\ldots \ldots \ldots \ldots \ldots \ldots \ldots \ldots \ldots \ldots \ldots$. 8 . 49

Light gray clay.................... $38 \quad 87$

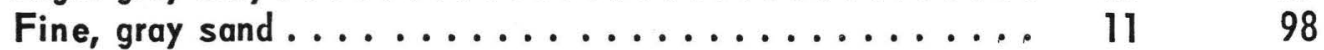

Red clay ..................... 3 101

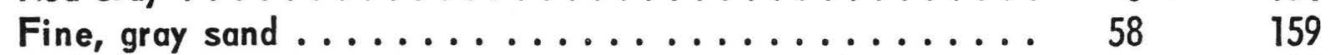

Red clay ........................ $28 \quad 187$

Fine to coarse, gray sand ................ 6

Sand ........................ $3 \quad 196$

Light gray clay ........................ $14 \quad 210$

Fine, gray, silty sand . . . . . . . . . . . . $12 \quad 222$

Gray clay ..................... $10 \quad 232$

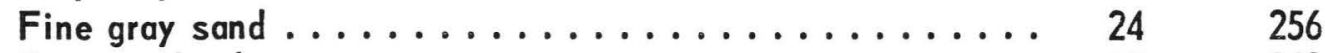

Gray sandy clay ..................... $12 \quad 268$

Early Paleozoic (?):

Wissahickon Formation:

Weathered rock ....................... 1269 
Table 3. - Selected drillers' logs of wells in Burlington County, N. J. - Continued

Well no. 26, Levitt and Sons Inc.

(Log by C. W. Lauman and Co. Inc.)

Quaternary:

Cape May Formation:

Brown sand and gravel .................. 11

Altitude 19 feet

Thickness

Depth

(feet) (feet)

Cretaceous:

Merchantville Formation:

Gray to black, sandy silt with lignite ... . . . . . . . 22

Magothy and Raritan Formations:

Fine to medium, gray sand ................. 14

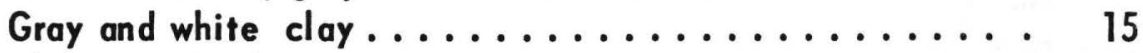

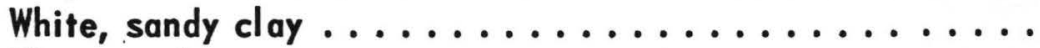

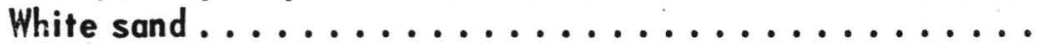

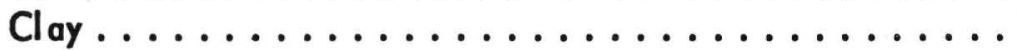

Red and white clayey sand $\ldots \ldots \ldots \ldots \ldots \ldots \ldots$

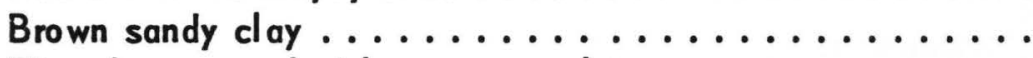

Fine, brown sand with some gravel ............

Fine, white, clayey sand and gravel ............ 5

Fine, light brown sand ...................... 10

Fine to coarse, brown sand with coarse gravel ........ 5

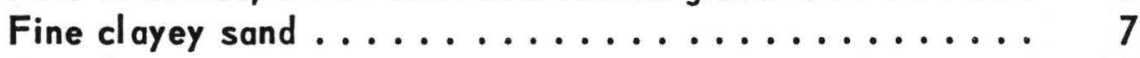

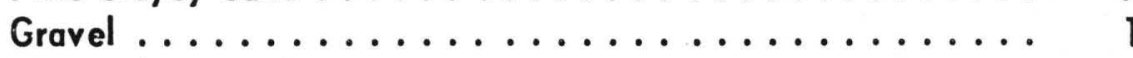

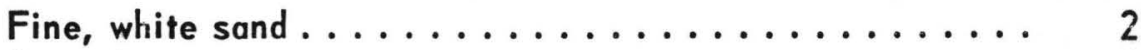

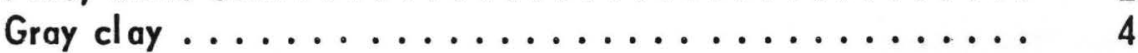

Fine to coarse, brown sand ................ 10

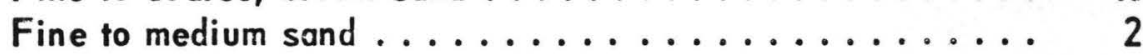

Clay ............................ 1

Fine to coarse, brown sand ............... 9

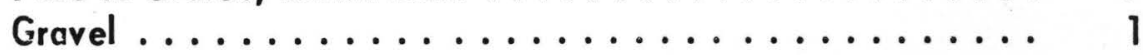

Red and gray clay ..................... 11

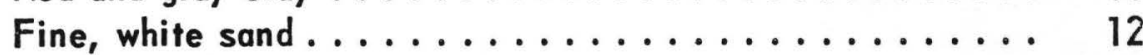

Fine to medium, brown sand . .............. 5

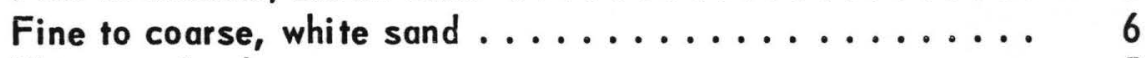

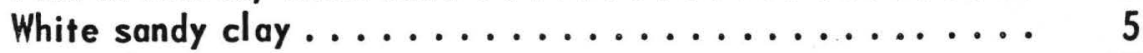

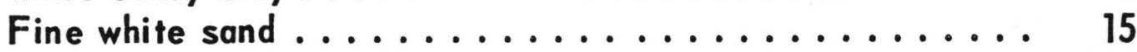

Fine gray sand with some clay ............... 10

Fine to coarse, gray sand ............... 7

Gray clay ........................ 3

Fine to coarse, gray sand ................ 6

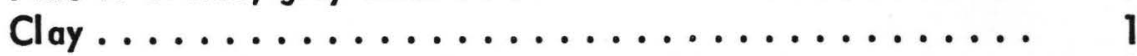

Fine to medium, gray sand $\ldots \ldots \ldots \ldots \ldots \ldots$

Fine to coarse, brown and white sand ............ 5

Fine to coarse, gray sand and gravel ............ 10

Gray clay ............................. 5

Red clay ............................. 6

Early Paleozoic (?):

Wissahickon Formation:

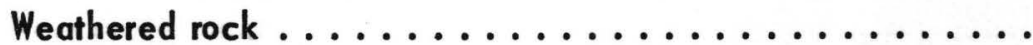


Table 3. - Selected drillers' logs of wells in Burlington County, N. J. - Continued

Well no. 27, Levitt and Sons Inc.

(Log by E. A. Hickok and D. W. Miller)

Quaternary:

Pensauken Formation:

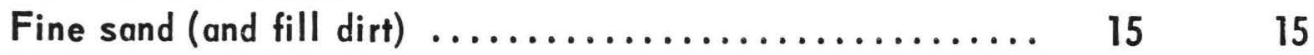

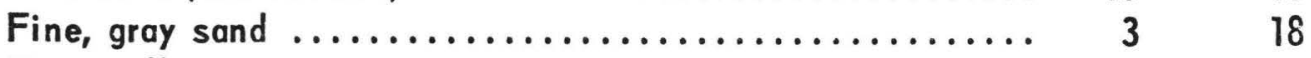

Fine, yellow sand....................... 20

Cretaceous:

Woodbury Clay:

Black mi caceous clay ...................... 30

Merchantville Formation:

Black to green glauconitic clay .................... $60 \quad 110$

Magothy (?) and Raritan (?) Formations:

Black to green glauconitic clay and some fine sand....... $10 \quad 120$

Magothy and Raritan Formations:

Gray and white clay ....................... $30 \quad 150$

Red and gray clay ........................... $10 \quad 160$

Gray silty clay ............................... $20 \quad 180$

Gray clay and medium gravel ...................... $10 \quad 190$

Red and gray clay........................... $30 \quad 220$

Dark gray clay with wood ...................... $10 \quad 230$

Gray clay ................................. $10 \quad 240$

Fine to medium sand with gray clay stringers ........... $20 \quad 260$

Fine to medium sand with some mica............... $10 \quad 270$

White silt................................... $10 \quad 280$

Sand, silt, and clay ......................... $10 \quad 290$

Gray clay ................................ $10 \quad 300$

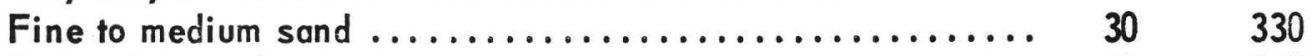

Red and gray clay ........................ $10 \quad 340$

Gray silt.............................. $10 . \ldots$

Fine to coarse sand witl gravel and clay............. $30 \quad 380$

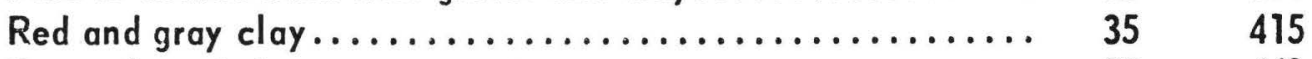

Gray silt and clay......................... $25 \quad 440$

Fine to medium sand ...................... $10 \quad 450$

Sand, gravel, and clay ...................... $10 \quad 460$

Gray clay and silt............................ $20 \quad 480$

Hedium to coarse sand and some gray clay ............ $10 \quad 490$

Gray clay with lignite ........................ 99

Medium gravel ............................. $10 \quad 509$

Gray clay and fine sand...................... 1

Early Paleozoic (?):

Wissahickon Formation:

Green to black, weathered, micaceous schist .......... $16 \quad 526$ 
Table 3. - Selected drillers' logs of wells in Burlington County, N. J. - Continued

Well no. 28, Lumberton Light, Water and Sewage Co.

(Log by Artesian Well Drilling Co.)

Cretaceous:

Altitude 10 feet

Thickness Depth

(feet) (feet)

Mount Laurel Sand and Wenonah Formation:

Sand........................................ 15

Undifferentiated:

Green marl and clay .......................... 69

Marshall town Formation:

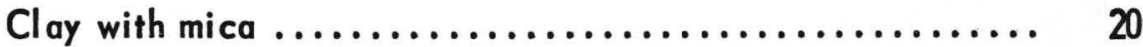

Englishtown Formation:

Black sand..................................... 20

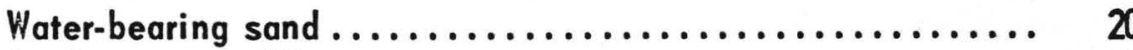

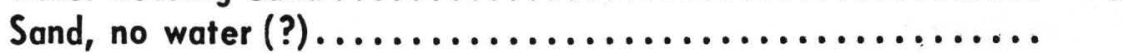

Woodbury Clay:

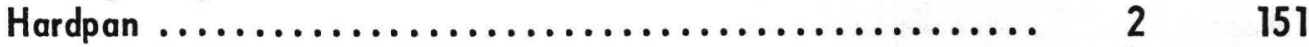

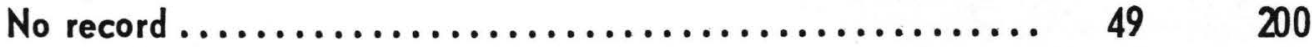

Black clay and mud ............................ $46 \quad 246$

Merchantville Formation:

Green marl ................................. $35 \quad 281$

Black marl ................................ $67 \quad 348$

Undifferentiated:

Marl and sand $\ldots \ldots \ldots \ldots \ldots \ldots \ldots \ldots \ldots \ldots \ldots \ldots \ldots, 12 \quad 360$

Magothy and Raritan Formations:

Pink clay..................................... 2362

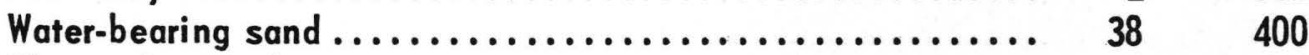

White, plastic clay ............................... 4404 
Table 3. - Selected drillers' logs of wells in Burlington County, N. J. - Continued

Well no. 29, Maple Shade Water Department

(Log by James M. McCrae L/)

Quaternary:

Altitude 55 feet

Thickness Depth

(feet) (feet)

Cape May Formation:

Surface gravel ...........................

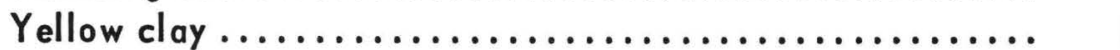

Cretaceous:

Woodbury $\mathrm{Cl}$ ay and Merchantville Formation (undifferentiated):

Fossiliferous, black sand ...................... 320

Black clay ............................... $44 \quad 64$

Magothy and Raritan Formations:

Fine to coarse, gray sand and gravel ............... $33 \quad 97$

Greensand marl (?) ............................... 603

Fine, gray sand and coarse gravel................. $27 \quad 130$

White clay ............................. $100 \quad 230$

Red clay................................ $10 \quad 240$

Sand and clay ................................ $20 \quad 260$

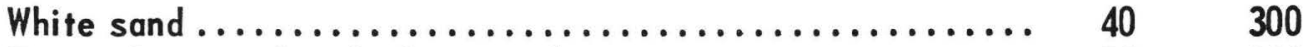

Fine, white sand with clay streaks ............... $15 \quad 315$

Coarse, white sand.......................... $35 \quad 350$

Coarse, white gravel ......................... $20 \quad 370$

Very coarse, white gravel with pebbles and boulders....... $5 \quad 375$

1/ Smock, 1894, p. 409.410 
Table 3. - Selected drillers' logs of wells in Burlington County, N. J. - Continued

Well no. 30, Maple Shade Water Department

(Log by Artesian Well Drilling Co.)

Quaternary:

Cape May Formation:

Clay and gravel............................... 8

Cretaceous:

Woodbury Clay and Merchantville Formation (undifferentiated):

Black marl ...................................... 63

Magothy and Raritan Formations:

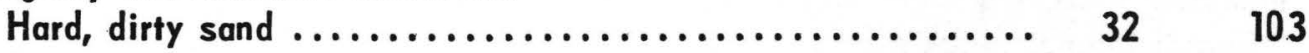

Sand and gravel $\ldots \ldots \ldots \ldots \ldots \ldots \ldots \ldots \ldots \ldots \ldots \ldots, 10,113$

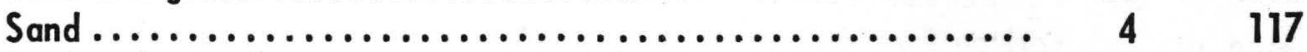

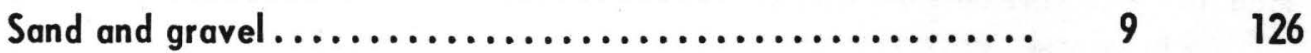

Sand and coarse gravel $\ldots \ldots \ldots \ldots \ldots \ldots \ldots \ldots \ldots \ldots, \quad 8 \ldots \ldots \ldots$

Clay .......................................... $38 \quad 172$

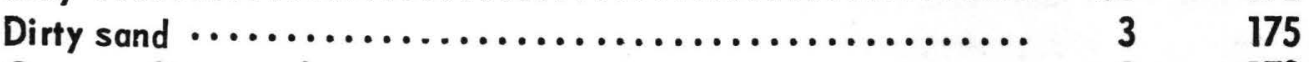

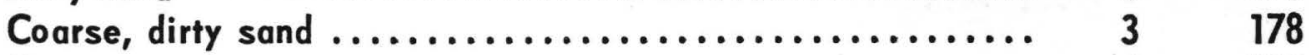

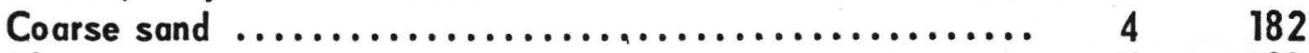

Clay ........................................ $7 \quad 189$

Fine dirty sand $\ldots \ldots \ldots \ldots \ldots \ldots \ldots \ldots \ldots \ldots \ldots \ldots, 7,196 \ldots \ldots$

Clay.......................................... $8 \quad 204$

Fine, dirty sand $\ldots \ldots \ldots \ldots \ldots \ldots \ldots \ldots \ldots \ldots \ldots \ldots \ldots \ldots \ldots \ldots, 11205$

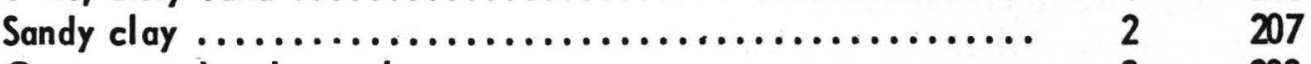

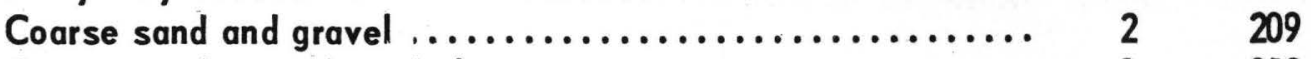

Coarse sand, gravel, and clay .................... 9218

Sandy clay ................................... $27 \quad 245$

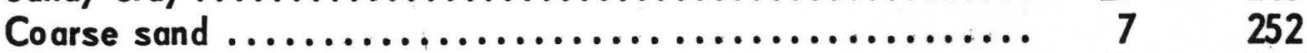

Clay ....................................... $20 \quad 272$

No sample $\ldots \ldots \ldots \ldots \ldots \ldots \ldots \ldots \ldots \ldots \ldots \ldots \ldots, 10,282$ 
Table 3. - Selected drillers' logs of wells in Burlington County, N. J. - Continued

Well no. 31, Medford Water Co.

(Log by Layne-New York Co., Inc.)

Topsoil

Quaternary:

Cape May Formation:

Yellow sandy clay and gravel..................... $11 \quad 12$

Tertiary:

Vincentown Formation and Hornerstown Sand (undifferentiated):

Green marl with shells and streaks of clay ............... 33

Tertiary and Cretaceous:

Undifferentiated:

Black sandy clay with shells $\ldots \ldots \ldots \ldots \ldots \ldots \ldots \ldots \ldots$. $39 \quad 84$

Fine sand with shells and streaks of gray clay .......... $27 \quad 111$

Cretaceous:

Mount Laurel Sand and Wenonah Formation:

Sandstone and shell s............................... $7 \quad 118$

Fine, gray sand with shells and streaks of clay........... $19 \quad 137$

Fine, black and white sand with shells ................ $13 \quad 150$

Marshall town Formation:

Black sandy clay with streaks of tough gray clay ......... $25 \quad 175$

Marshall town and Englishtown Formations (undifferentiated):

Gray sandy clay with shells ....................... 84

Englishtown Formation:

Tough, gray clay ............................ $6 \quad 265$

Woodbury Clay:

Gray sandy clay and shells $\ldots \ldots \ldots \ldots \ldots \ldots \ldots \ldots \ldots . .6252$

Tough, gray clay .............................. $15 \quad 332$

Fine, black and white sand with shells .............. $26 \quad 358$

Merchantville Formation:

Black marl with shells and streaks of gray clay.......... $77 \quad 435$

Magothy and Raritan Formations:

Hard, black and white sand with shells and streaks

of gray clay .................................. $59 \quad 494$

Tough, gray clay.............................. 6500

Fine to medium, white sand $\ldots \ldots \ldots \ldots \ldots \ldots \ldots \ldots \ldots, 18 \ldots \ldots, 518$

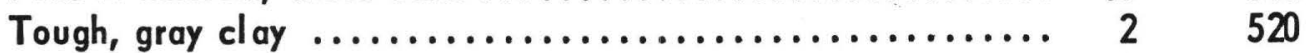

Medium to coarse, gray sand $\ldots \ldots \ldots \ldots \ldots \ldots \ldots \ldots \ldots, 20 \quad 540$

White sandy clay $\ldots \ldots \ldots \ldots \ldots \ldots \ldots \ldots \ldots \ldots \ldots \ldots, 12 \quad 552$

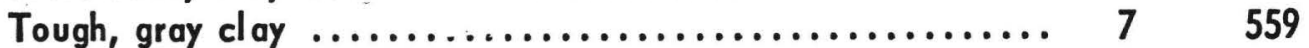

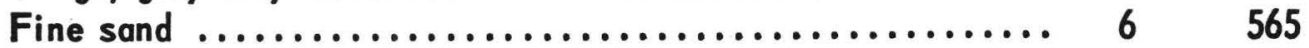

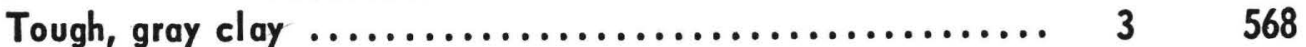

Hard sand with streaks of gray clay ................. $22 \quad 590$ 
Table 3. - Selected drillers' logs of wells in Burlington County, N. J. - Continued Well no. 32, Moorestown Township Water Department (Log by Layne-New York Co., Inc.)

Cretaceous:

$\begin{array}{cc}\text { Altitude } 20 \text { feet } \\ \text { Thickness } & \text { Depth } \\ \text { (feet) } & \text { (feet) }\end{array}$

Woodbury $\mathrm{Cl}$ ay and Merchantville Formation (undifferentiated):

Elue sandy clay $\ldots \ldots \ldots \ldots \ldots \ldots \ldots \ldots \ldots \ldots, 13$

Elue clay................................ 112

Magothy and Faritan Formations:

Fine, gray sand $\ldots \ldots \ldots \ldots \ldots \ldots \ldots \ldots \ldots \ldots \ldots \ldots \ldots \ldots, 23 \quad 148$

Qlue clay ................................ $8 \quad 156$

Coarse, gray sand $\ldots \ldots \ldots \ldots \ldots \ldots \ldots \ldots \ldots \ldots \ldots, 33 \quad 189$

Mhite clay ................................. $60 \quad 249$

Coarse, gray sand $\ldots \ldots \ldots \ldots \ldots \ldots \ldots \ldots \ldots \ldots \ldots \ldots$. $56 \quad 305$

Blue clay ................................ 15,320

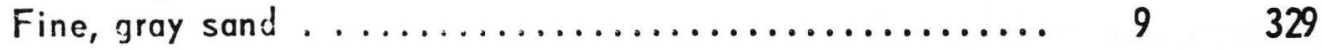

Blue clay ................................ 8

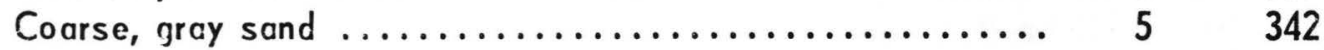

Coarse, gray sand and gravel $\ldots \ldots \ldots \ldots \ldots \ldots \ldots \ldots \ldots . \quad 33 \quad 375$

Yellow clay................................... 738

Red clay .................................... $34 \quad 416$

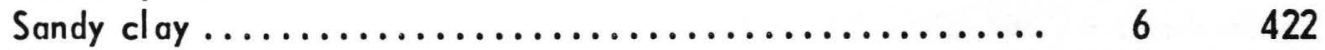

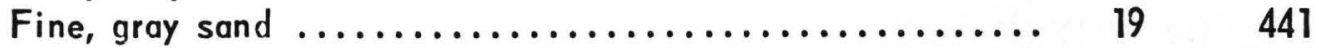

Coarse sand $\ldots \ldots \ldots \ldots \ldots \ldots \ldots \ldots \ldots \ldots \ldots \ldots \ldots . \ldots \ldots, 22 \quad 463$

Sand, gravel, and boulders ..................... $31 \quad 494$

Blue clay ............................... 2496

Early Paleozoic (?):

Wissahickon Formation:

Rock..................................... I 497 
Table 3. - Selected drillers' logs of wells in Burlington County, N. J. - Continued

Well no. 34, Mount Holly Water Company

(Log by John C. Smock 2) )

Quaternary:

Cape May Formation:

Surface material $\ldots \ldots \ldots \ldots \ldots \ldots \ldots \ldots \ldots \ldots \ldots \ldots, 12$

Cretaceous:

Mount Laurel Sand and Wenonah Formation:

Dark gray micaceous sand with some green sand (glauconite)

Altitude 20 feet

Thickness Depth

(feet) (feet)

Marshall town Formation:

Marly clay ................................... $\quad 45$

At 12

Englishtown Formation:

Water-bearing, yellow sand $\ldots \ldots \ldots \ldots \ldots \ldots \ldots \ldots \ldots, 40,97$

Woodbury Clay:

Dark sandy clay .............................. $25 \quad 122$

Clay with gravel $\ldots \ldots \ldots \ldots \ldots \ldots \ldots \ldots \ldots \ldots \ldots \ldots \ldots$ At 122

Dark marly clay .............................. $71 \quad 193$

Merchantville Formation:

Green sand (glauconite) marl ...................... $42 \quad 235$

Coarse, dark sandy clay ......................... $40 \quad 275$

Magothy (?) and Raritan Formations (?):

Pink, very clayey sand ........................... $15 \quad 290$

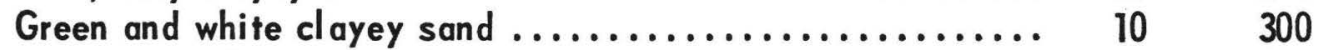

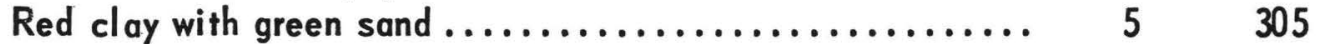

Clayey sand and green sand (glauconite) .............. $15 \quad 320$

Magothy and Raritan Formations:

Water-bearing, white lignitic sand .................. $20 \quad 340$

Fine, yellow gravel .............................. 2342

Dark, micaceous sandy clay ........................ $8 \quad 350$

White micaceous sand ............................ $18 \quad 368$

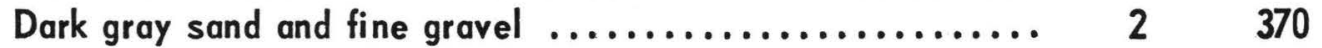

Green clayey sand and fine gravel $\ldots . \ldots . \ldots . \ldots . \ldots . \ldots . .24 \quad 394$

Sandstone ..................................... At 394

Pink sandy clay .............................. $106 \quad 500$

Red cl ay, less sandy ........................... $39 \quad 539$

Water-bearing sand and gravel $\ldots \ldots \ldots \ldots \ldots \ldots \ldots \ldots . \quad 23 \quad 562$

2/ Smock, 1901, p. 144-147 
Table 3. - Selected drillers' logs of wells in Burlington County, N. J. - Continued Well no. 35, Pemberton Water Department (Log by A. L. Lyons)

Tertiary:

Altitude 80 feet Thickness Depth (feet) (feet)

Manasquan Formation:

Yellow sandy clay ............................ $17 \quad 17$

Vincentown Formation:

Gray sand $\ldots \ldots \ldots \ldots \ldots \ldots \ldots \ldots \ldots \ldots \ldots \ldots \ldots, 22$

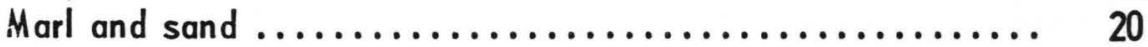

Undifferentiated:

Green sand and clay

Tertiary and Cretaceous:

Hornerstown Sand and Navesink Formation (undifferentiated):

Blue clay and marl

Cretaceous:

Mount Laurel Sand and Wenonah Formation:

Dark gray sand

Marshall town Formation:

Dark sandy clay

Well no. 37, Pemberton Township Water Department (Log by Thomas Magee)

Tertiary:

Cohansey Sand:

Sand

Kirkwood Formation:

White sandy clay $\ldots \ldots \ldots \ldots \ldots \ldots \ldots \ldots \ldots \ldots \ldots, 7$

Yellow sandy clay ............................. $\quad 40$

Altitude 112 feet

White sand ...

Black marl ...

Manasquan and Vincentown Formations, Hornerstown Sand (undifferentiated):

Green marl .....................................

Green sandy marl

Cretaceous:

Navesink Formation:

Hard, green marl

Green sandy mar

Green sandy marl with shell

$14 \quad 210$

Mount Laurel Sand and Wenonah Formation:

Hard sand

Hard sand and marl with shells

27 
Table 3. - Selected drillers' logs of wells in Burlington County, N. J. - Continued

Well no. 38, Pemberton Township Water Department

(Log by Layne-New York Co., Inc.)

$\quad \begin{gathered}\text { Altitude } 93 \text { feet } \\ \text { Thickness } \\ \text { (feet) }\end{gathered}$
Depth
(feet)


Table 3. - Selected drillers' logs of wells in Burlington County, N. J. - Continued

Well no. 39, Rancocas Woods Water Co. (Log by Charles L. Mollitor Inc.)

Topsoil

$$
\text { (Log by Charles L. Mollitor Inc.) }
$$

\begin{tabular}{|c|c|c|}
\hline & $\begin{array}{c}\text { Altitude } 5 \\
\text { Thickness } \\
\text { (feet) }\end{array}$ & $\begin{array}{l}0 \text { feet } \\
\text { Depth } \\
\text { (feet) }\end{array}$ \\
\hline Topsoil .......... & . 1 & \\
\hline $\begin{array}{l}\text { retaceous: } \\
\text { Englishtown Formation: }\end{array}$ & & \\
\hline Sand $\ldots \ldots \ldots \ldots$ & 20 & 21 \\
\hline Water-bearing, fine sand $\ldots \ldots \ldots \ldots \ldots \ldots \ldots \ldots \ldots$ & 12 & \\
\hline $\begin{array}{l}\text { Brown sand and gravel } \ldots \ldots \ldots \ldots \ldots \ldots \ldots \ldots \ldots \ldots \ldots \ldots \ldots \\
\text { Woodbury Clay: }\end{array}$ & 27 & 60 \\
\hline 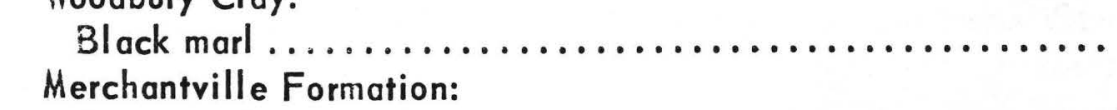 & 60 & 120 \\
\hline 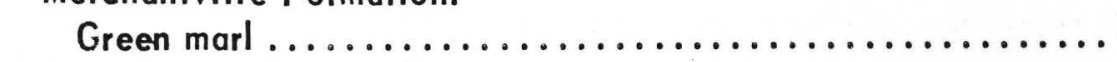 & 60 & 180 \\
\hline Magothy and Faritan Formations: & & \\
\hline White clay ........... & 7 & 187 \\
\hline & 48 & 235 \\
\hline Sand and gravel $\ldots \ldots \ldots \ldots \ldots \ldots \ldots \ldots \ldots$ & 13 & 248 \\
\hline
\end{tabular}

Well no. 40, Riverton and Palinyra Water Co. (Log by A. C. Schultes and Sons)

Altitude 13 feet

Topsoil Quaternary:

Cape May Formation:

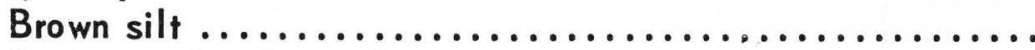

Brown sand and gravel $\ldots \ldots \ldots \ldots \ldots \ldots \ldots \ldots \ldots \ldots \ldots$

Cretaceous:

Magothy and Raritan Formations:

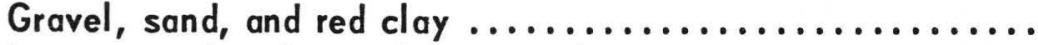

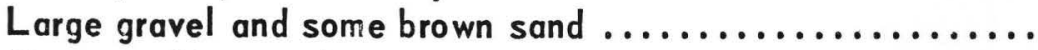

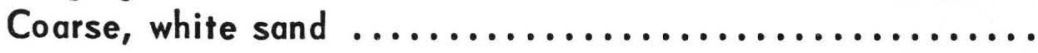

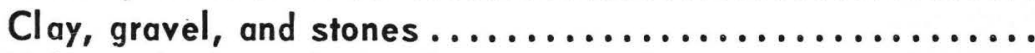

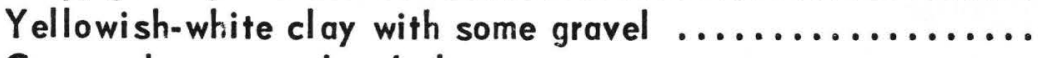

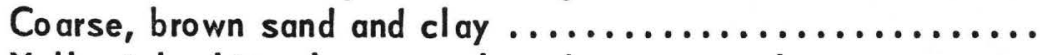

Yellowish-white clay, gravel, and coarse sand ...........

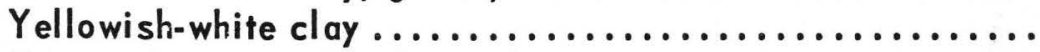

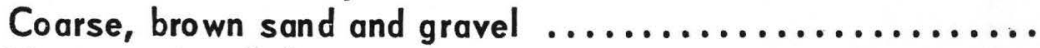

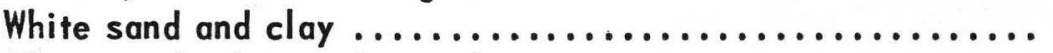

White sand, clay, and gravel $\ldots \ldots \ldots \ldots \ldots \ldots \ldots \ldots$

White sand with some gravel $\ldots \ldots \ldots \ldots \ldots \ldots \ldots \ldots \ldots$

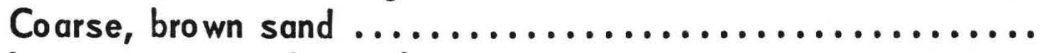

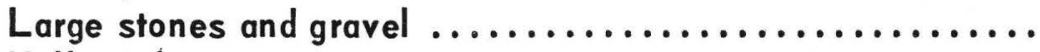

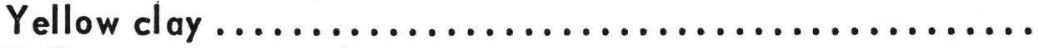

Yellow mi caceous clay ......................... 3

$\begin{array}{rr}1 & 1 \\ 8 & 9 \\ 9 & 18\end{array}$

$\begin{array}{rr}2 & 20 \\ 14 & 34 \\ 1 & 35 \\ 6 & 41 \\ 6 & 47 \\ 11 & 58 \\ 1 & 59 \\ 3 & 62 \\ 7 & 69 \\ 4 & 73 \\ 1 & 74 \\ 5 & 79 \\ 2 & 81 \\ 3 & 84 \\ 9 & 93 \\ 3 & 96\end{array}$

Early Pal eozoic (?):

Wissahickon Formation:

Weathered rock 
Table 3. - Selected drillers' logs of wells in Burlington County, N. J. - Continued

Well no. 42, Norman F. Scattergood

(Log by Theodore Ziegenfus)

Cuaternary:

Altitude 72 feet

Thickness Deptli

(feet) (feet)

Cape May Formation:

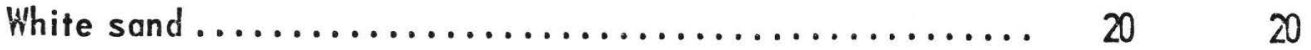

Cretaceous:

Undifferentiated:

Blue clay................................ $125 \quad 145$

Magothy and Faritan Formations:

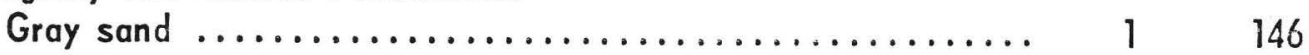

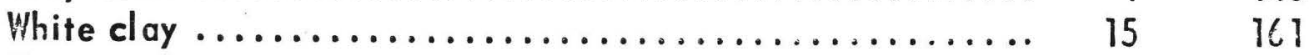

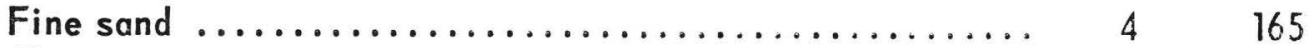

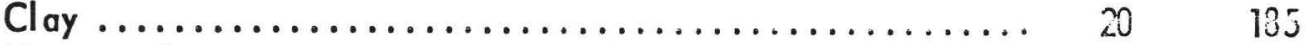

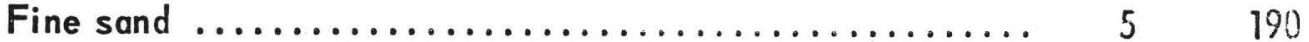

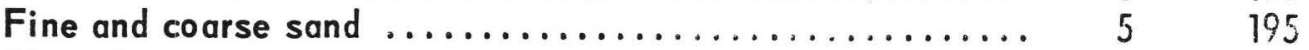

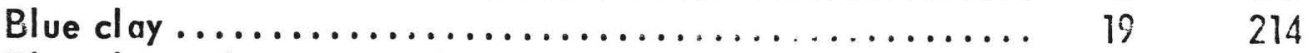

Blue clay and coarse sand .................... 6220

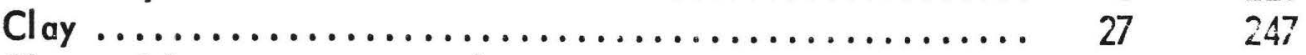

Clay and fine to coarse sand .................. 6 . 253

Fed clay ................................ 10 26 263

White clay ................................. $16 \quad 279$

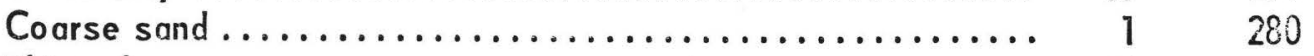

Vihite clay ........................................ 35

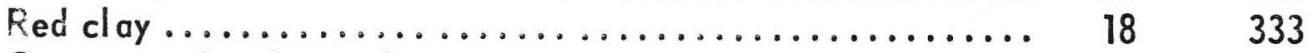

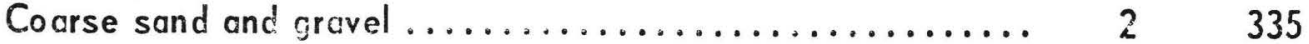

Dlue and white clay ......................... 13

Fine to coarse sand and grcvel ................. $14 \quad 362$ 
Table 3. - Selected drillers' logs of wells in Burlington County, N. J. - Continued Well no. 44, National Guard Maintenance Shop (Log by Louis M. Bainbridge and Son)

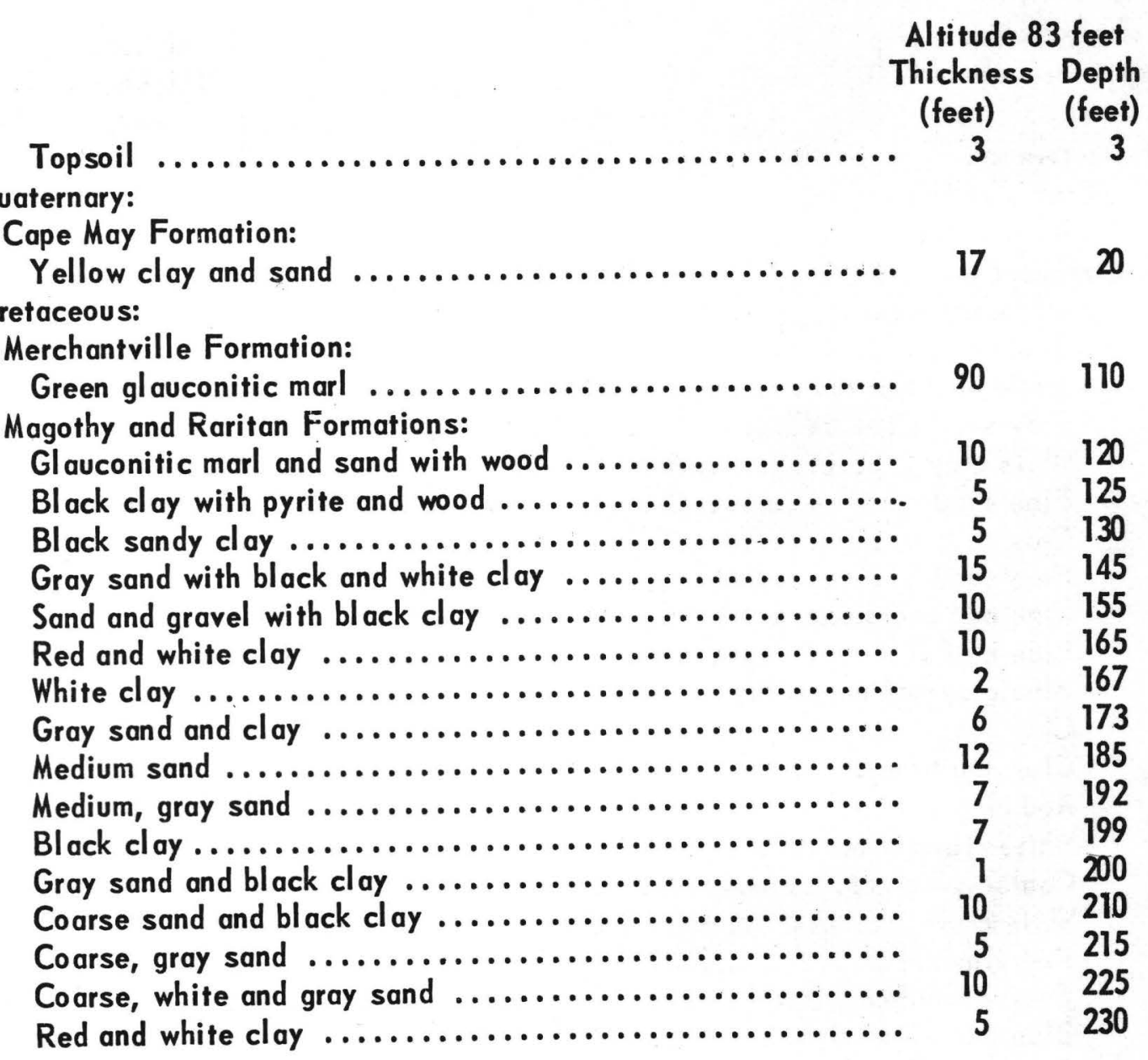

Well no. 45, Hercules Powder Co.

(Log by Layne-New York Co., Inc.)

Quaternary:

Altitude 22 feet

Cape May Formation:

Sand.

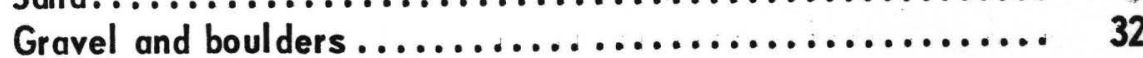

Cretaceous:

Magothy and Raritan Formations:

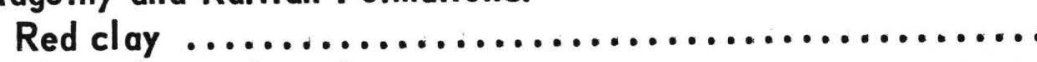

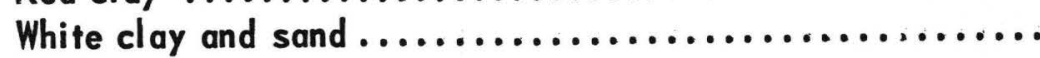

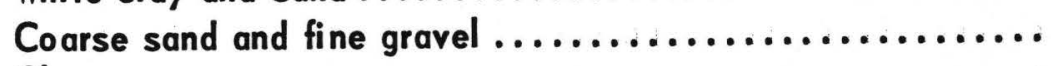

Clay ..............................................

Sand and gravel

$39 \quad 77$

19.96

$24 \quad 120$

$7 \quad 127$

$8 \quad 135$

Early Pal eozoic (?):

Wissahickon Formation:

Mica rock 
Table 3. - Selected drillers' logs of wells in Burlington County, N. J. - Continued

Well no. 48, Edgar B. Heisler

(Log by Charles L. Mollitor Inc.)

Fill

Altitude 30 feet

Thickness Depth

(feet)

(feet)

Quaternary:

Cape May Formation:

Dry sand ..............

Cape May (?) Formation:

Yellow sand

18

19

Cretaceous:

Magothy and Faritan Formations:

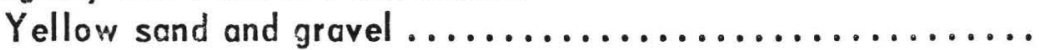

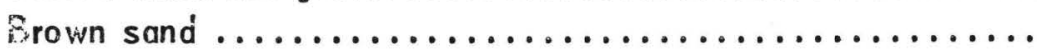

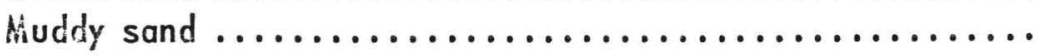

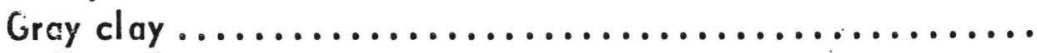

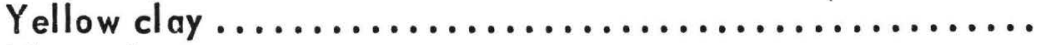

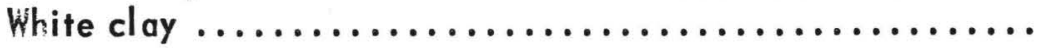

Yellow sand $\ldots \ldots \ldots \ldots \ldots \ldots \ldots \ldots \ldots \ldots \ldots \ldots \ldots \ldots$

Yellow sand and clay $. . \ldots \ldots \ldots \ldots \ldots \ldots \ldots \ldots \ldots . . . \ldots \ldots$

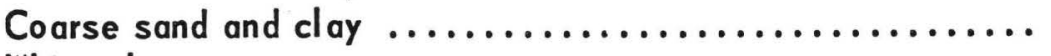

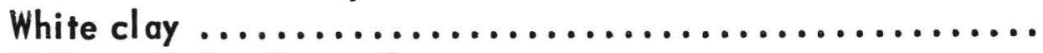

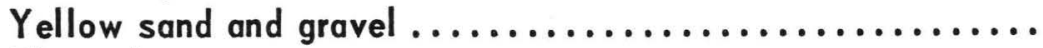

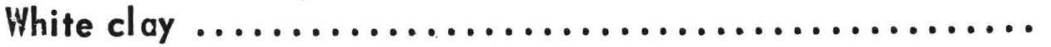

Sand and gravel $\ldots \ldots \ldots \ldots \ldots \ldots \ldots \ldots \ldots \ldots \ldots$

Well no. 49, Carroll Murphy

(Log by Charles L. Mollitor Inc.)

Quaternary:

Cape May Formation:

Altitude 35 feet

Medium to coarse, brown sand and loam ............... Medium to coarse sand

Cretaceous:

Magothy and Raritan Formations:

Gray clay .................................... 22

White clay with sand.......................... 725

Red clay with sand $\ldots \ldots \ldots \ldots \ldots \ldots \ldots \ldots \ldots \ldots \ldots \ldots \ldots$. 5 . $30 . \ldots$

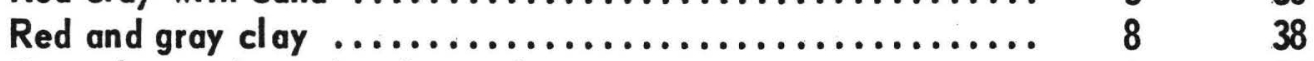

Gray clay with sand and gravel ..................... 34

Brown sandstone .......................... 10.51

Medium to coarse, gray sand $\ldots \ldots \ldots \ldots \ldots \ldots \ldots \ldots \ldots \ldots, 25$

Fine, white sand $\ldots \ldots \ldots \ldots \ldots \ldots \ldots \ldots \ldots \ldots \ldots \ldots \ldots, 6 \ldots \ldots$

White clay and white sand $\ldots \ldots \ldots \ldots \ldots \ldots \ldots \ldots \ldots \ldots .1183$ 
Table 3. - Selected drillers' logs of well's in Burlington County, N. J. - Continued

Well no. 50, Masonic Home

(Log by American Well Drilling Co.)

Quaternary:

Pensauken Formation:

Yellow sandy clay

Cretaceous:

Woodbury Clay and Merchantville Formation (undifferentiated):

Black silty clay

Altitude 79 feet

Thickness Depth

(feet) (feet)

Hagothy and Raritan Formations:

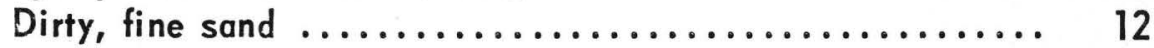

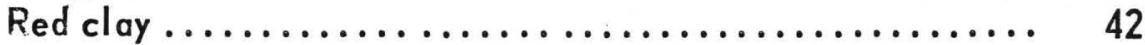

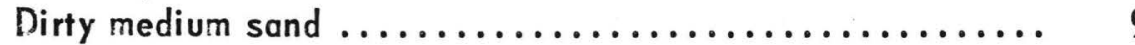

Clean, fine to coarse, gray sand ................... 13

Fine to coarse, gray sand with wood fragments $\ldots \ldots \ldots \ldots$

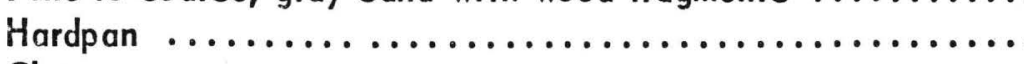

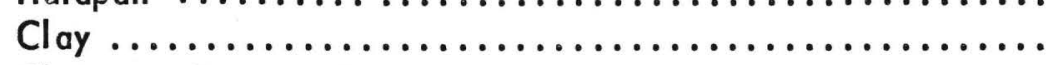

Clean, medium sand.$\ldots \ldots \ldots \ldots \ldots \ldots \ldots \ldots \ldots \ldots$

Clean, coarse sand and fine gravel $\ldots \ldots \ldots \ldots \ldots \ldots \ldots$

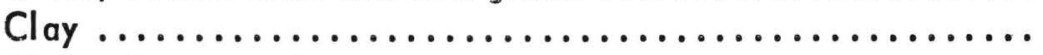

Clean, fine gravel $\ldots \ldots \ldots \ldots \ldots \ldots \ldots \ldots \ldots \ldots \ldots \ldots$

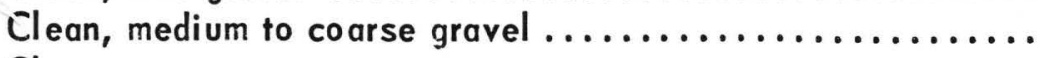

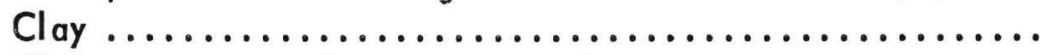

Clean, coarse sand.$\ldots \ldots \ldots \ldots \ldots \ldots \ldots \ldots \ldots \ldots \ldots$

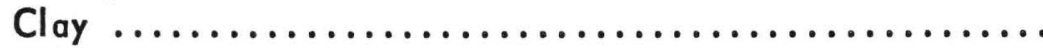

Clean, coarse sand and fine gravel $\ldots \ldots \ldots \ldots \ldots \ldots \ldots \ldots$

Red and white clay $\ldots \ldots \ldots \ldots \ldots \ldots \ldots \ldots \ldots \ldots$

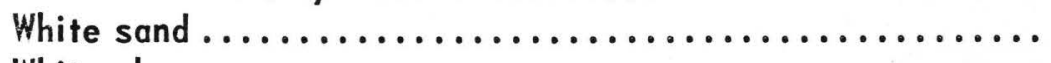

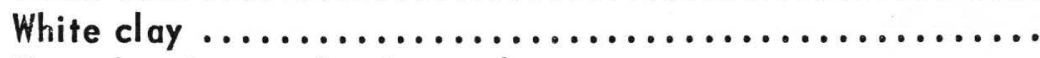

Water-bearing sand and gravel $\ldots \ldots \ldots \ldots \ldots \ldots \ldots \ldots$

Red clay 
Table 3. - Selected drillers' logs of wells in Burlington County, N. J. - Continued

Well no. 51, William A. Donald

(Log by Paul Steffens)

Loamy topsoil

Altitude 42 feet

Thickness Depth

(feet) (feet)

Quaternary:

Cape May Formation:

Clay 6 6

Dirty sand

Cretaceous (?):

Magothy (?) and Raritan (?) Formations:

Sand and gravel

Cretaceous:

Magothy and Raritan Formations:

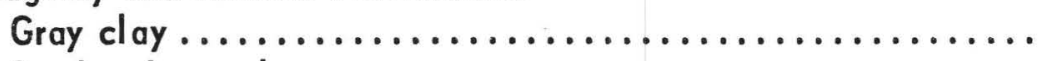

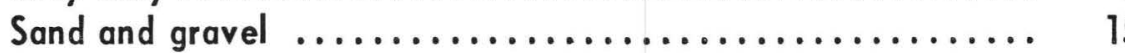

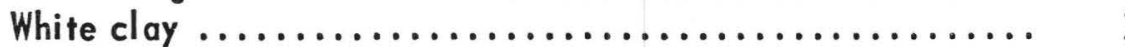

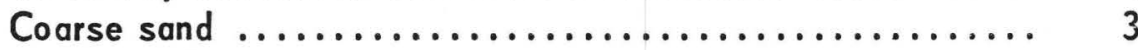

Fine gravel $\ldots \ldots \ldots \ldots \ldots \ldots \ldots \ldots \ldots \ldots \ldots \ldots \ldots \ldots, 1$

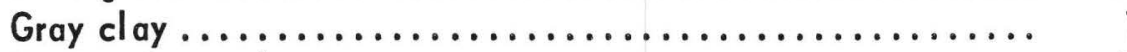

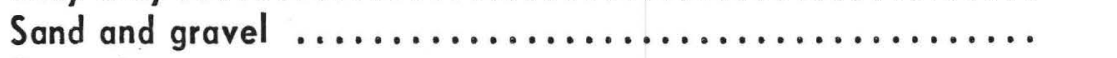

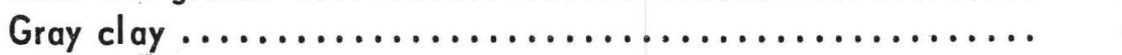

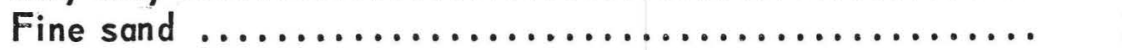

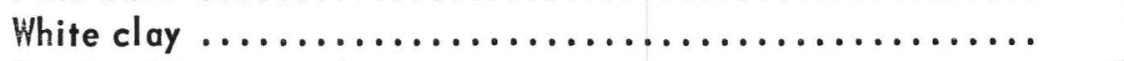

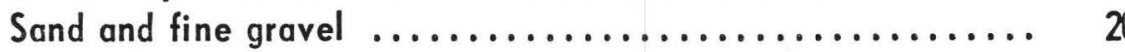

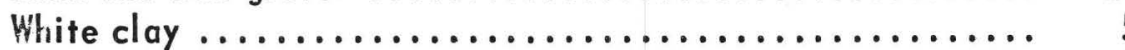

Englishtown Formation:

Brown loam ....................................

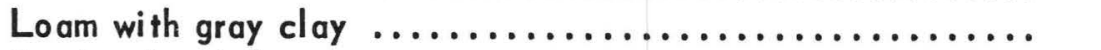

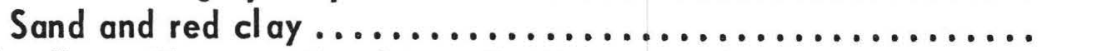

Woodbury Clay and Merchantville Formation (undifferentiated):

Black and gray clay ............................ 85

Green marl with hardpan .......................... 27

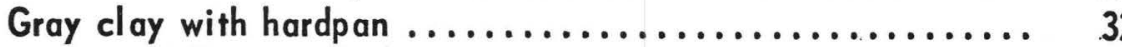

Magothy and Raritan Formations:

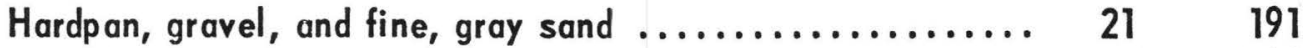

Water-bearing, fine, gray sand .................... $17 \quad 208$ 
Table 3. - Selected drillers' logs of wells in Burlington County, N. J. - Continued Well no. 55, Frank Pino (Log by Edward Robbins)

Cretaceous (?):

Merchantville (?) Formation:

Altitude 63 feet

Thickness Depth

(feet) (feet)

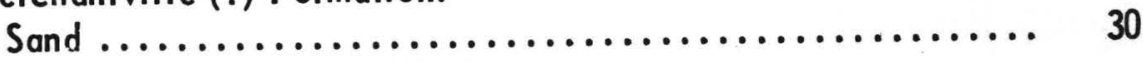

Magothy (?) and Raritan (?) Formations:

Light-gray clay...

Cretaceous:

Magothy and Raritan Formations:

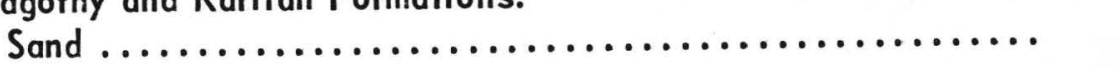

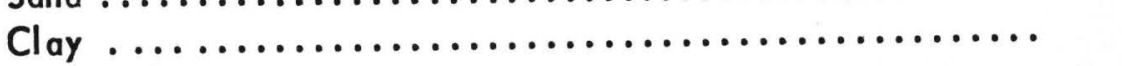

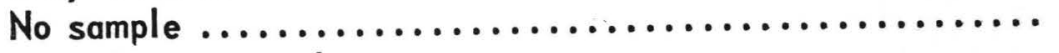

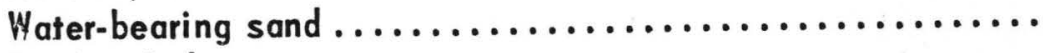

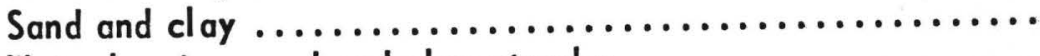

Water-bearing sand and clay streaks $\ldots \ldots \ldots \ldots \ldots \ldots \ldots$

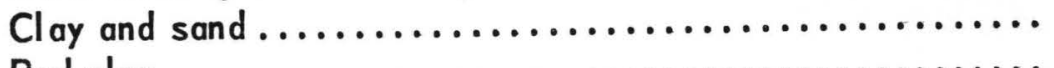

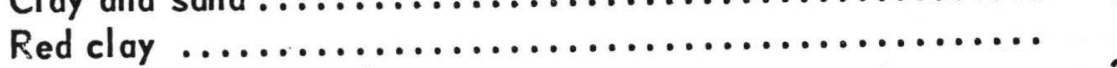

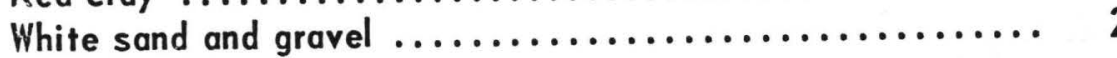

30

$50 \quad 80$

81

182

890

$10 \quad 100$

$49 \quad 149$

$20 \quad 169$

$31 \quad 200$

$7 \quad 207$

$20 \quad 227$

Well no. 56, National Gypsum Co.

(Log by Layne-New York Co., Inc.)

Topsoil

Quaternary:

Cape May Formation:

Red sand and clay ...

Brown sand and gravel ................................

Coarse sand and gravel ........................ 10

Altitude 22 feet

$11 \quad 21$

$10 \quad 31$

Cretaceous:

Magothy and Raritan Formations:

White and yellow sandy clay.

Coarse, yellow sand and gravel with streaks of yellow clay.

Yellow and white sandy clay with some gravel ...............

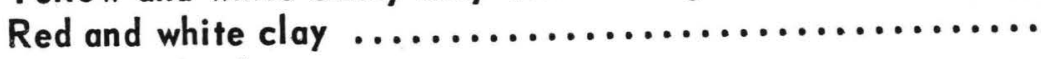

White sandy clay

Brown sand and coarse gravel .........................

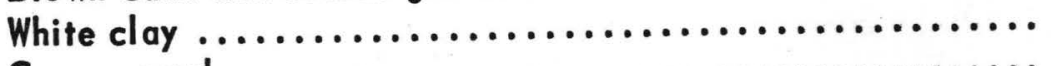

Coarse sand

White and yellow clay 
Table 3. - Selected drillers' logs of wells in Burlington County, N. J. - Continued

Well no. 58, Charles Heal, Jr.

(Log by Charles L. Mollitor Inc.)

Quaternary:

Altitude 50 feet

Thickness Depth

(feet) (feet)

Cape May Formation:

Sand

Dry sand

$1 \quad 1$

Sand

$4 \quad 5$

Gravel

$14 \quad 19$

Cretaceous:

Merchantville Formation:

Black marl

2

21

Magothy and Raritan Formations:

Sand ...

Gravel

71

980

Well no. 61, Harrisville

(Log by Mahlon Broon $\underline{3}$ )

Tertiary:

Altitude 15 feet

Cohansey Sand:

Sand.

77

Sand.

Blue mud

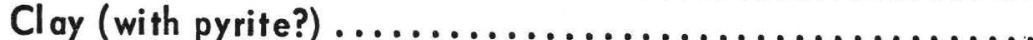

Marly mud...

108

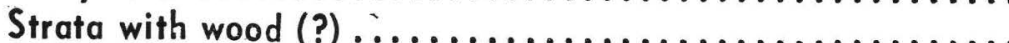

Mud with shells

Hard sediments

Water-bearing sand

196

Dark, slushy sand

231

Yellow sand

245

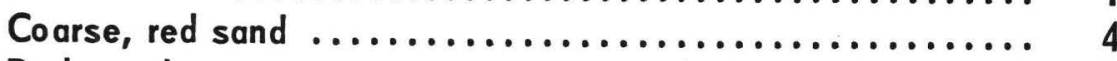

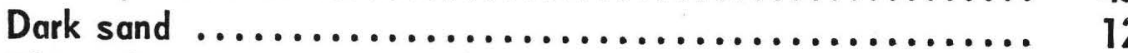

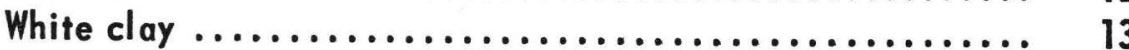

Kirkwood Formation:

Water-bearing, green marl $\ldots \ldots \ldots \ldots \ldots \ldots \ldots \ldots \ldots \ldots . .37 \quad 368$

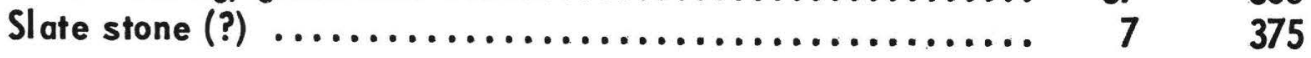

3/ Smock, 1893, p. 288-289 
Table 3. - Selected drillers' logs of wells in Burlington County, N. J. - Continued

Well no. 62, New Jersey State Reformatory

(Log by Layne-New York Co., Inc.)

Topsoil

Altitude 97 feet

Thickness Depth

(feet)

1

(feet)

Quaternary:

Pensauken Formation:

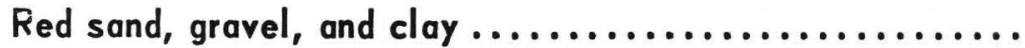

Cretaceous:

Woodbury Clay and Merchantville Formation (undifferentiated):

Tough, black clay ............................. 22

Soft, green clay ............................... 40

35

Soft, black clay

$40 \quad 115$

Magothy (?) and Raritan (?) Formations:

Soft, gray clay

140

Magothy and Raritan Formations:

Fine, white sand.............................. 35

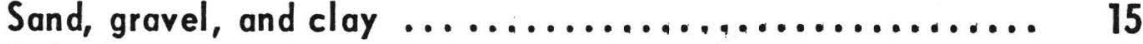

Soft clay ...................................... 15

Coarse, white sand $\ldots \ldots \ldots \ldots \ldots \ldots \ldots \ldots \ldots \ldots \ldots . \ldots \ldots$

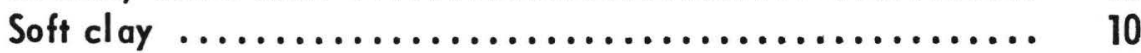

Sand with streaks of clay $\ldots \ldots \ldots \ldots \ldots \ldots \ldots \ldots \ldots \ldots .22$

Clay ......................................... 12

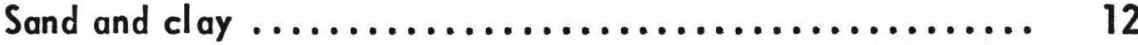

12

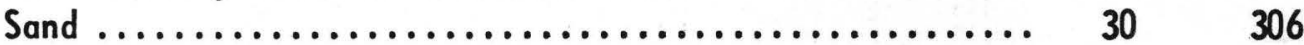

Fed clay ................................... $39 \quad 345$

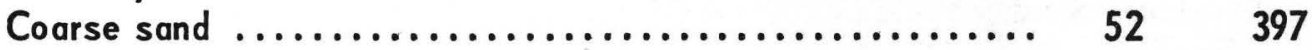

Clay ..................................... $25 \quad 422$

Coarse sand $\ldots \ldots \ldots \ldots \ldots \ldots \ldots \ldots \ldots \ldots \ldots \ldots \ldots \ldots \ldots . \ldots 2244$

Clay ......................................... 10 
Table 3. - Selected drillers' logs of wells in Burlington County, N. J. - Continued

Well no. 66, Dana Packing Co.

(Log by Greenhalgh and Kaye)

Quaternary (?):

Altitude 208 feet

Thickness Depth

(feet) (feet)

Bridgeton (?) Formation:

Yellow gravel ............

$38 \quad 38$

Tertiary and Cretaceous:

Hornerstown and Red Bank Sands, Navesink Formation

(undifferentiated):

Dense, brown clay .............................. $22 \quad 60$

Green and black marl ........................... $46 \quad 106$

Cretaceous (?):

Mount Laurel (?) Sand and Wenonah (?) Formation:

Green clay..................................... $24 \quad 130$

Dark gray clay $\ldots \ldots \ldots \ldots \ldots \ldots \ldots \ldots \ldots \ldots \ldots \ldots \ldots, \quad 25 \quad 155$

Cretaceous:

Marshall town Formation:

Clay and fine sand $\ldots \ldots \ldots \ldots \ldots \ldots \ldots \ldots \ldots \ldots, 95,250$

Englishtown Formation:

Coarse, yellow sand $\ldots \ldots \ldots \ldots \ldots \ldots \ldots \ldots \ldots \ldots, 26 \ldots \ldots$

Well no. 67, Francis D. Gray

(Log by Louis M. Bainbridge and Son)

Cretaceous:

Altitude 92 feet

Mount Laurel Sand and Wenonah Formation:

Yellow sand and clay ........................ 30

30

Marshall town Formation:

Green clay ................................... 15

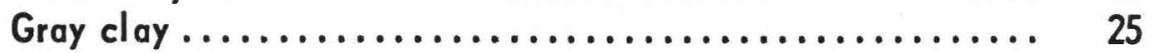

45

Englishtown Formation:

Gray sand with pyrite ........................ $16 \quad 86$

Gray clay ................................ 5

Gray sand and clay ......................... $10 \quad 101$

Englishtown Formation, Woodbury Clay and Merchantville

Formation (undifferentiated):

Gray clay ................................. $94 \quad 195$

Green marl ................................ $40 \quad 235$

Gray clay ........................................ $25 \quad 260$

Grayish-green clay ........................... $5 \quad 265$

Black clay .................................. $14 \quad 279$

Magothy and Raritan Formations:

Gray sand and clay ............................ $34 \quad 313$

Black clay .................................... At 313

Gray sand ................................ $4 \quad 317$ 
Table 3. - Selected drillers' logs of wells in Burlington County, N. J. - Continued

Well no. 68, John Marincas

(Log by Greenhalgh and Kaye)

Altitude 97 feet

Thickness Depth

(feet) (feet)

Quaternary and Cretaceous:

Pensauken and Englishtown Formations (undifferentiated):

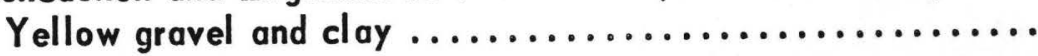

Cretaceous:

Woodbury Clay and Merchantville Formation (undifferentiated):

Dense, black clay

Gray and black clay.

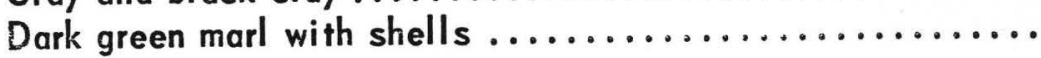

Black clay

45

15

Magothy and Raritan Formations:

$\mathrm{Cl}$ ay and sand

Coarse sand and sandstone with wood

Hell no. 70, Hoeganaes Sponge Iron Co.

(Log by Layne-New York Co., Inc.)

Quaternary:

Cape May (?) Formation:

Sand

Cretaceous:

Magothy and Faritan Formations:

Sand and sandy clay

Muddy sand

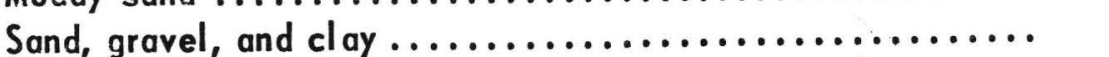

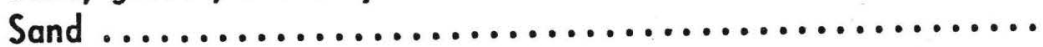

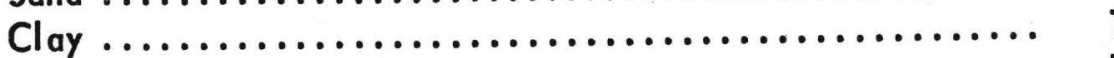

Sand, gravel, and clay ........................

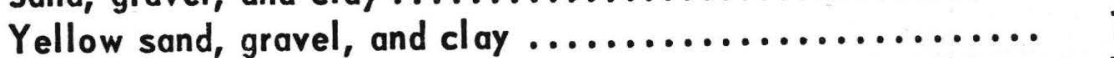

Gray sand and white clay ........................

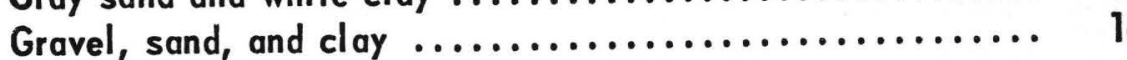

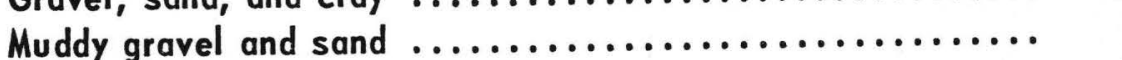

Gravel with streaks of clay $\ldots . \ldots \ldots \ldots \ldots \ldots \ldots \ldots \ldots$

Coarse sand and gravel ......................... 26 
Table 3. - Selected drillers' logs of wells in Burlington County, N. J. - Continued Well no. 71, Eastampton School (Log by Charles L. Mollitor, Inc.)

\begin{tabular}{|c|c|c|}
\hline & $\begin{array}{l}\text { Altitude } 7 \\
\text { Thickness } \\
\text { (feet) }\end{array}$ & $\begin{array}{l}3 \text { feet } \\
\text { Depth } \\
\text { (feet) }\end{array}$ \\
\hline $\begin{array}{l}\text { Loamy topsoil } \ldots \ldots \ldots \ldots \ldots \ldots \ldots \ldots \ldots \ldots \ldots \ldots \ldots \ldots \ldots \ldots \ldots \ldots \\
\text { etaceous: } \\
\text { Mount Laurel Sand and Wenonak Formation: }\end{array}$ & . 6 & 6 \\
\hline 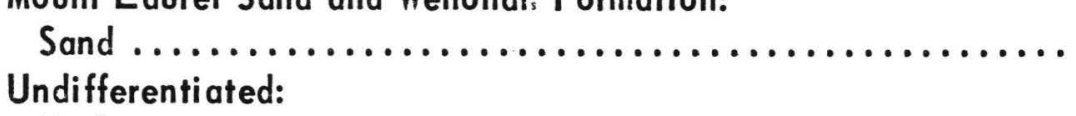 & 8 & 14 \\
\hline 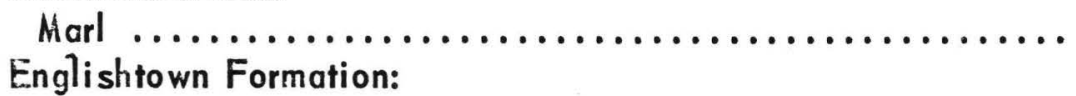 & 117 & 131 \\
\hline 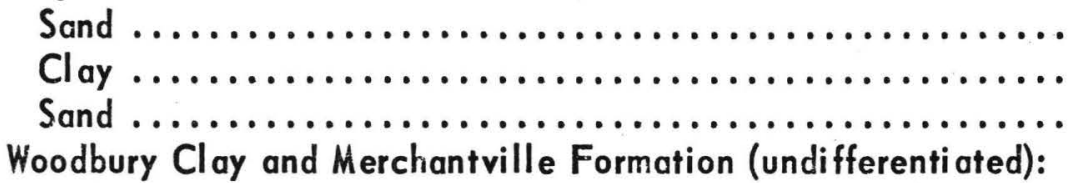 & $\begin{array}{l}15 \\
23 \\
13\end{array}$ & $\begin{array}{l}146 \\
169 \\
182\end{array}$ \\
\hline Magothy and Raritan Formations: & 161 & 343 \\
\hline 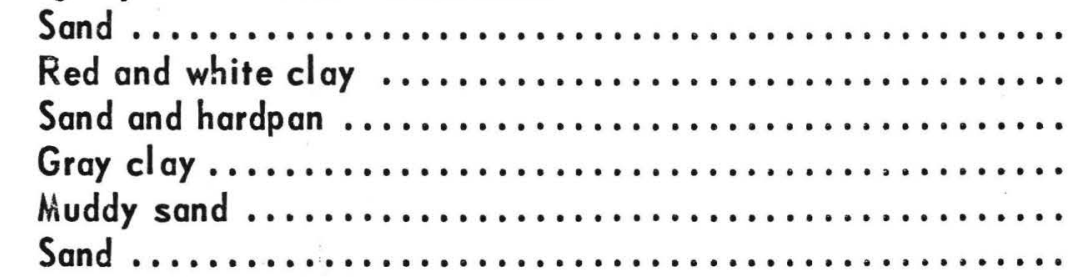 & $\begin{array}{r}4 \\
22 \\
5 \\
21 \\
6 \\
16\end{array}$ & $\begin{array}{l}347 \\
369 \\
374 \\
395 \\
401 \\
417\end{array}$ \\
\hline
\end{tabular}


Table 3. - Selected drillers' logs of wells in Burlington County, N. J. - Continued Well no. 72, J. A. Methvin

(Log by Paul Steffens)

Loamy top soil

Altitude 39 feet

Thickness Depth

(feet)

(feet)

Cretaceous:

11

11

Mount Laurel Sand and Wenonah and Marshalltown Formations

(undifferentiated):

Black sandy marl

Englishtown Formation:

Water-bearing, fine sand

Dark gray clay

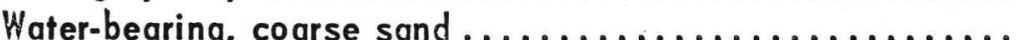

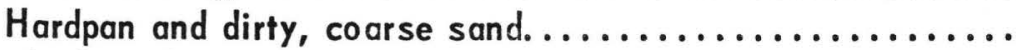

Black marl

Fine sand with wood

Black marl

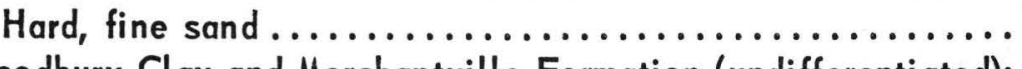

Woodbury $\mathrm{Cl}$ ay and Merchantville Formation (undifferentiated):

Very stiff, black marl $\ldots \ldots \ldots \ldots \ldots \ldots \ldots \ldots \ldots \ldots \ldots$

Marl with streaks of hardpan $\ldots \ldots \ldots \ldots \ldots \ldots \ldots \ldots \ldots$

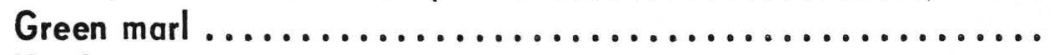

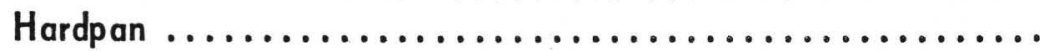

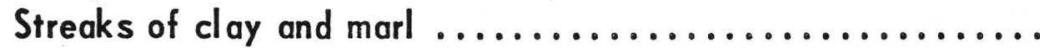

Sandy marl with thin streaks of sand $\ldots \ldots \ldots \ldots \ldots \ldots$

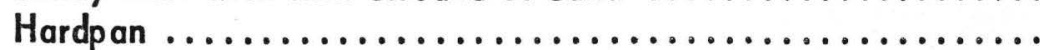

Mar

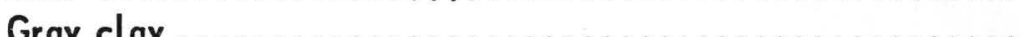

Magothy and Raritan Formations:

Hard sand .................................. 4

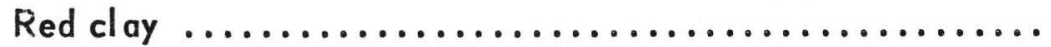

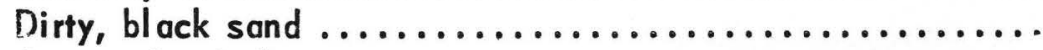

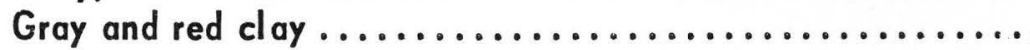

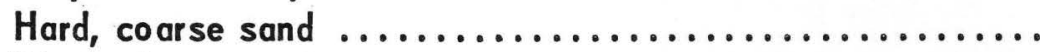

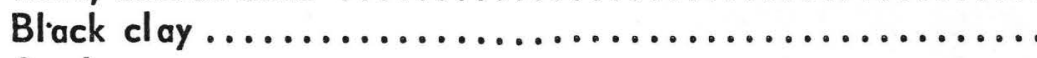

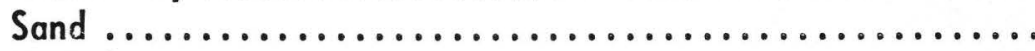

Fed clay 
Table 3. - Selected drillers' logs of wells in Burlington County, N. J. - Continued

Well no. 73, Byron T. Roberts Farms, Inc.

(Log by A. C. Schultes and Sons)

Cretaceous:

Altitude 93 feet

Thickness Depth

Navesink Formation:

Fine to medium, brown sand $\ldots \ldots \ldots \ldots \ldots \ldots \ldots \ldots \ldots$. 6 . 6

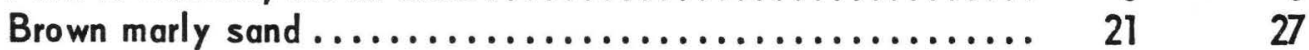

Gray marl .................................... 55

Mount Laurel Sand and Wenonah Formation:

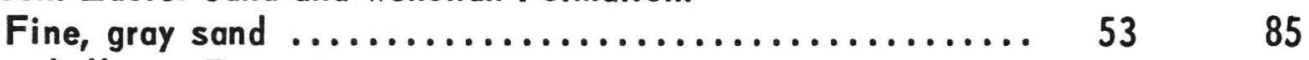

Marshall town Formation:

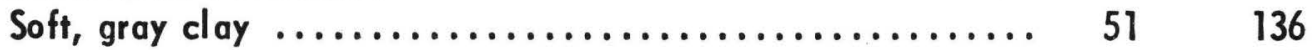

Gray, laminated clay........................... $4 \quad 140$

Englishtown Formation:

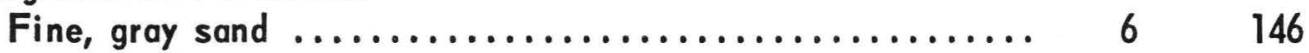

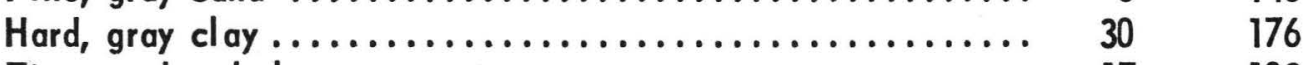

Fine sand and clay .......................... $17 \quad 193$

Woodbury Clay and Merchantville Formation (undifferentiated):

Gray clay.................................. $79 \quad 272$

Hard, gray clay .............................. $43 \quad 315$

Magothy and Raritan Formations:

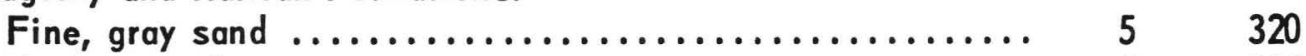

Hard, gray clay ............................... $2 \quad 322$

Fine to coarse, gray sand $\ldots \ldots \ldots \ldots \ldots \ldots \ldots \ldots \ldots \ldots, 32 \quad 354$

Clay ........................................ $6 \quad 360$

Fine sand $\ldots \ldots \ldots \ldots \ldots \ldots \ldots \ldots \ldots \ldots \ldots \ldots \ldots, \quad 22 \quad 382$

Hard, gray clay $\ldots \ldots \ldots \ldots \ldots \ldots \ldots \ldots \ldots \ldots \ldots \ldots, 24 \quad 406$ 
Table 3. - Selected drillers' logs of wells in Burlington County, N. J. - Continued Well no. 74, David D. Griscom

(Log by J. Henry Robbins)

Quaternary:

Pensauken Formation:

Yellow clay ................................. 20

Cretaceous:

Navesink Formation:

Black mud ... 50

Undifferentiated:

Green marl ..................................... 80

Altitude 92 feet

Thickness Depth

(feet) (feet)

Hard, black clay ............................. 120

Green clay .....................................

Magothy (?) and Raritan (?) Formations:

Black and gray clay ............................ 20

Magothy and Raritan Formations:

White sand...

Well no. 76, Earl R. Blyler

(Log by Charles L. Mollitor, Inc.)

Topsoil

Quaternary:

Cape May Formation:

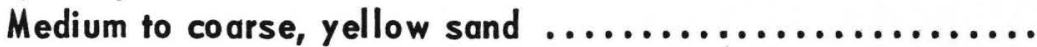

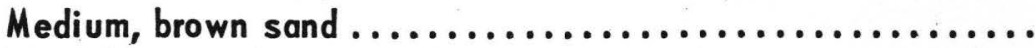

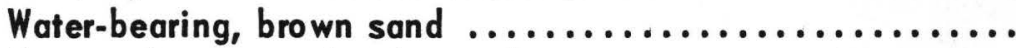

Coarse, brown sand and gravel....................

Altitude 39 feet

Very coarse sand and gravel

150

270

30300

320

340

Cretaceous:

Magothy and Raritan Formations:

Grayish-white sandy clay .......................... 10

Fine, yellow sand, clay, and hardpan ................. 2

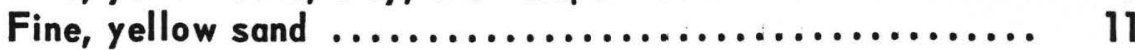

Medium to coarse, dark yellow sand $\ldots . . . \ldots \ldots \ldots \ldots . . . .6$

White clay with iron-stained sand $\ldots \ldots \ldots \ldots \ldots \ldots \ldots \ldots . .2$

White clay, sand, and hardpan ......................

Water-bearing, medium to coarse, yellow sand ............

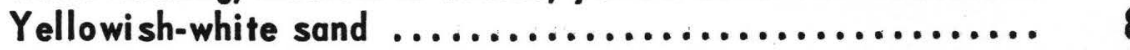

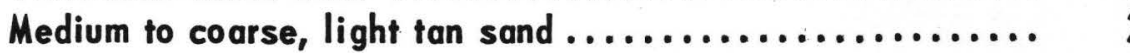

Coarse, light tan sand with clay ...................... 10

Water-bearing, medium to coarse, light tan sand and gravel ....................................... 
Table 3. - Selected drillers' logs of wells in Burlington County, N. J. - Continued

Well no. 78, Amico Sand and Gravel Co.

(Log by Paul Steffens)

Quaternary:

Cape May Formation:

Sand

Coarse gravel

Altitude 15 feet

Thickness Depth

(feet) (feet)

Cretaceous:

Magothy (?) and Raritan (?) Formations:

Black clay ......................................

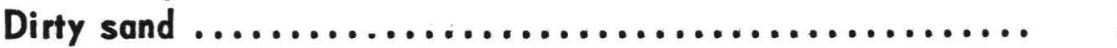

Sand and gravel $\ldots \ldots \ldots \ldots \ldots \ldots \ldots \ldots \ldots \ldots \ldots \ldots, 12$

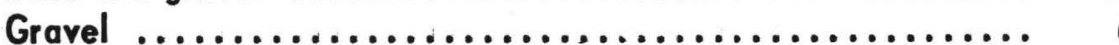

Clay ............................................ 13

3

arly Paleozoic (?):

Wissahickon Formation:

Soft rock

Well no. 80, Holy Cross High School

(Log by Charles L. Mollitor Inc.)

Altitude 82 feet

Topsoil

Cretaceous:

Magothy and Raritan Formations:

Yellow sand

Brown sand

4

White clay

Red clay

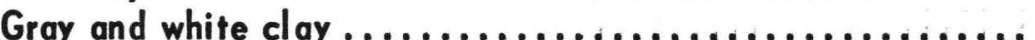

Gray clay

Dirty, gray st

White clay

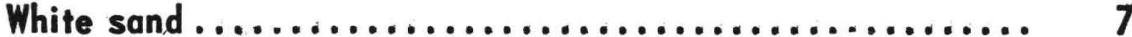

White clay ......................................

Sand and gravel

153

$21 \quad 174$ 
Table 3. - Selected drillers' logs of wells in Burlington County, N. J. - Continued Well no. 81, Holiday Lake (Log by Charles L. Mollitor, Inc.)

Quaternary (?):

Altitude 25 feet
Thickness $\begin{gathered}\text { Depth } \\ \text { (feet) }\end{gathered}$

Cape May (?) Formation:

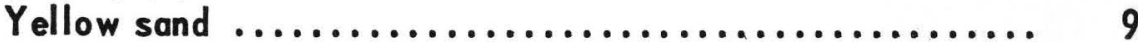

Sand $\ldots \ldots \ldots \ldots \ldots \ldots \ldots \ldots \ldots \ldots \ldots \ldots \ldots \ldots, 12$

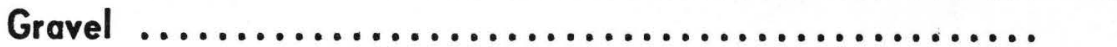

$4 \quad 25$

Cretaceous:

Magothy and Raritan Formations:

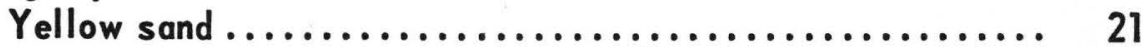

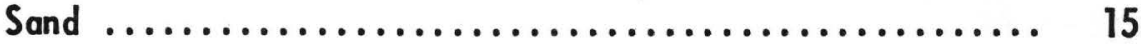

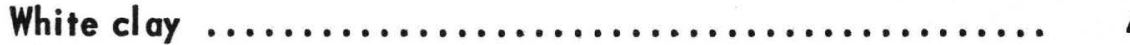

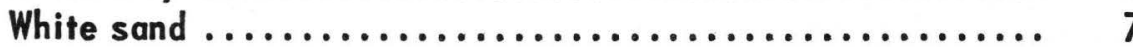

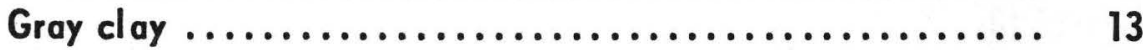

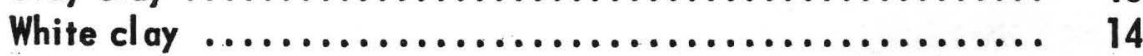

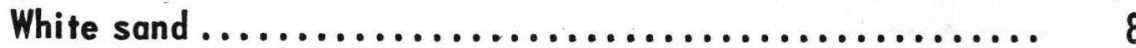

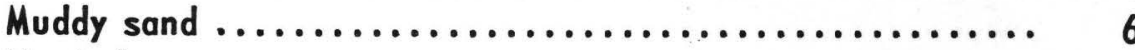

Hard clay ..................................... 6

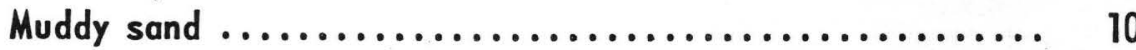

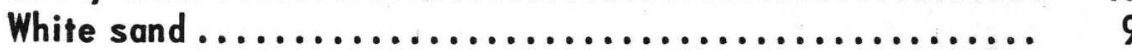

White clay ......................................

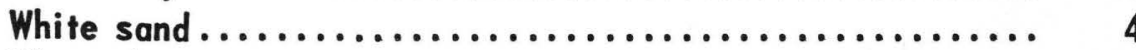

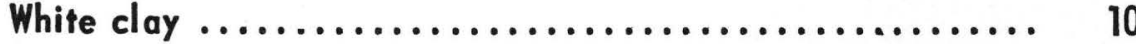

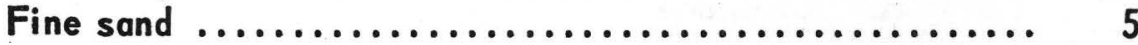

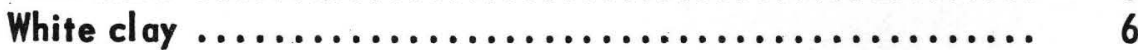

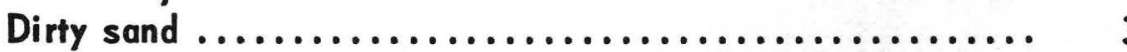

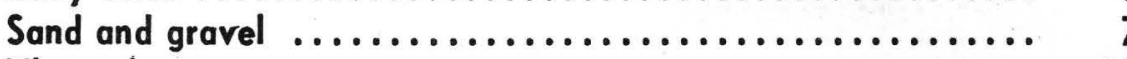

White clay ..................................... 10

Sand and gravel 
Table 3. - Selected drillers' logs of wells in Burlington County, N. J. - Continued

Well no. 88, John H. Cook

(Log by Charles L. Mollitor Inc.)

Topsoil

Quaternary:

Pensauken Formation:

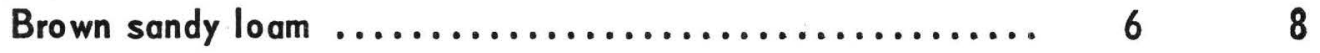

Cretaceous:

Mount Laurel Sand and Wenonah Formation:

Hard, coarse, brown sand with some clay ............. 7

Hard, brown sandy clay ....................... $16 \quad 31$

Marshall town (?) Formation:

Green marl ................................ $14 \quad 45$

Black clay .............................. $25 \quad 70$

Marshalltown Formation:

Black to green marl ........................... 6

Gray sandy clay ............................ $16 \quad 92$

Black clay with some fine sand................ $40 \quad 132$

Englishtown Formation:

Water-bearing, green sand with fossils and wood ........ $16 \quad 148$ 
Table 3. - Selected drillers' logs of wells in Burlington County, N. J. - Continued Well no. 89, Turnpike Junction Industrial Park (Log by A. C. Schultes and Sons)

Altitude 60 feet Thickness Depth

(feet) (feet)

Cretaceous:

Woodbury Clay and Merchantville Formation (undifferentiated):

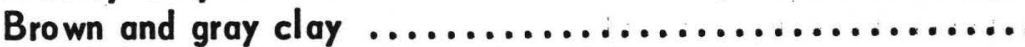

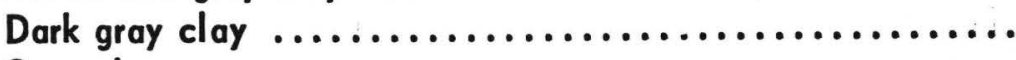

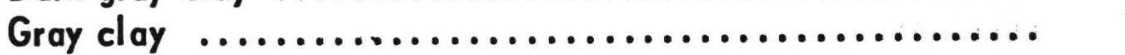

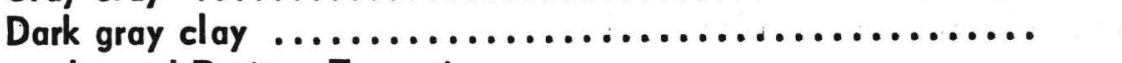

Magothy and Raritan Formations:

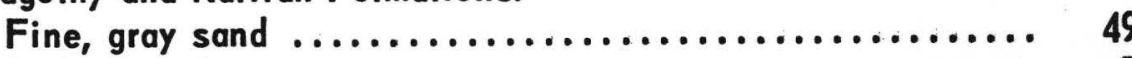

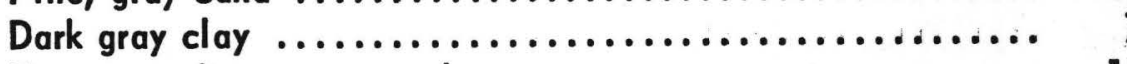

Fine to medium, gray sand $. . \ldots \ldots \ldots \ldots \ldots \ldots \ldots \ldots . .15$

Dark gray clay ..................................

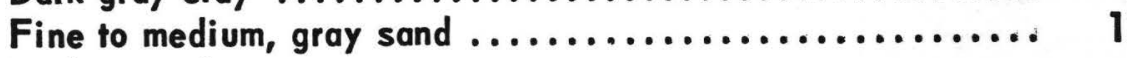

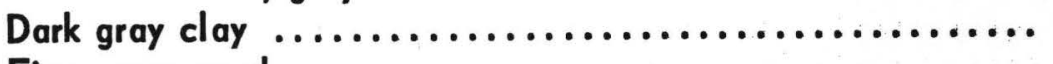

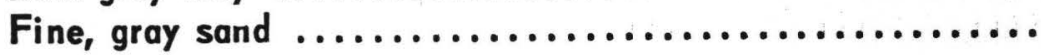

Red clay ........................................

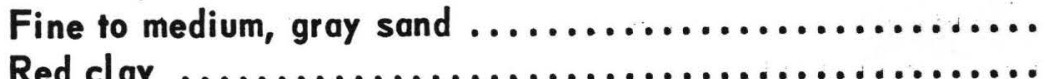

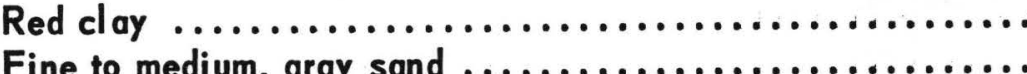

Red clay .......................................

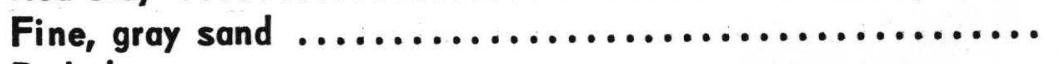

Red clay .......................................

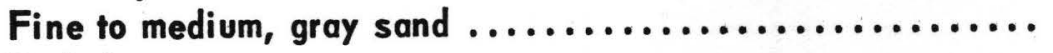

Red clay ........................................

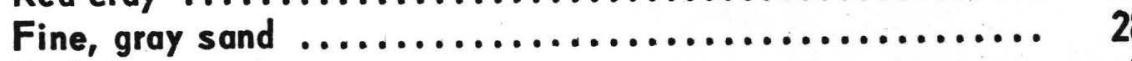

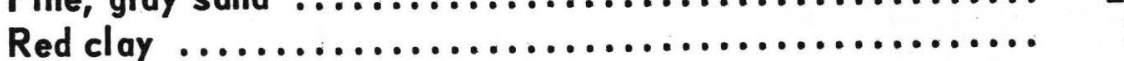

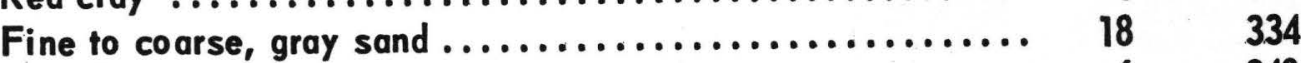

Red clay 
Table 3. - Selected drillers' logs of wells in Burlington County, N. J. - Continued

Well no. 90, Franklin Tallman

(Log by Charles L. Mollitor Inc.)

Quaternary:

Cape May Formation:

Fine, brown sand and loam

Altitude 82 feet

Thickness Depth

(feet) (feet)

retaceous (?):

Englishtown (?) Formation:

Hardpan .................................. 1

Medium, yellow sand $\ldots \ldots \ldots \ldots \ldots \ldots \ldots \ldots \ldots \ldots \ldots \ldots \ldots . .77$

Water-bearing, medium sand with a thin layer of hardpan $\ldots . .47$

Brown clay .............................. 9

Cretaceous:

Woodbury Clay and Merchantville Formation (undifferentiated):

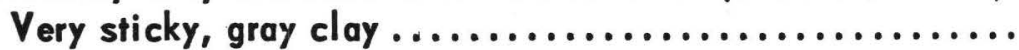

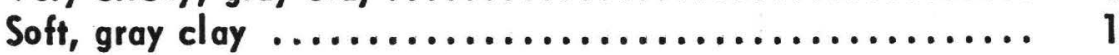

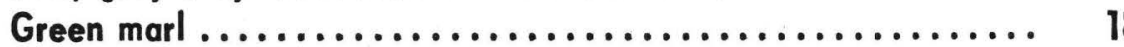

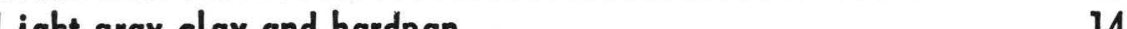

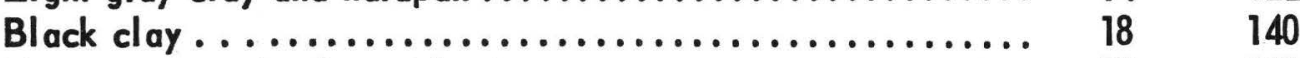

Fine, gray sandy clay with mica ................... $20 \quad 160$

Hard, black clay ............................ $20 \quad 180$

Magothy and Raritan Formations:

Red and white sandy clay ....................... 7

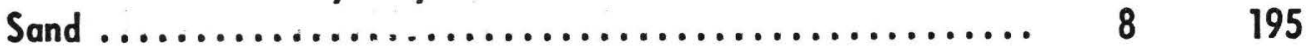


Table 3. - Selected drillers' logs of wells in Burlington County, N. J. - Continued Well no. 91 , Ed Phares (Log by Louis Bainbridge)

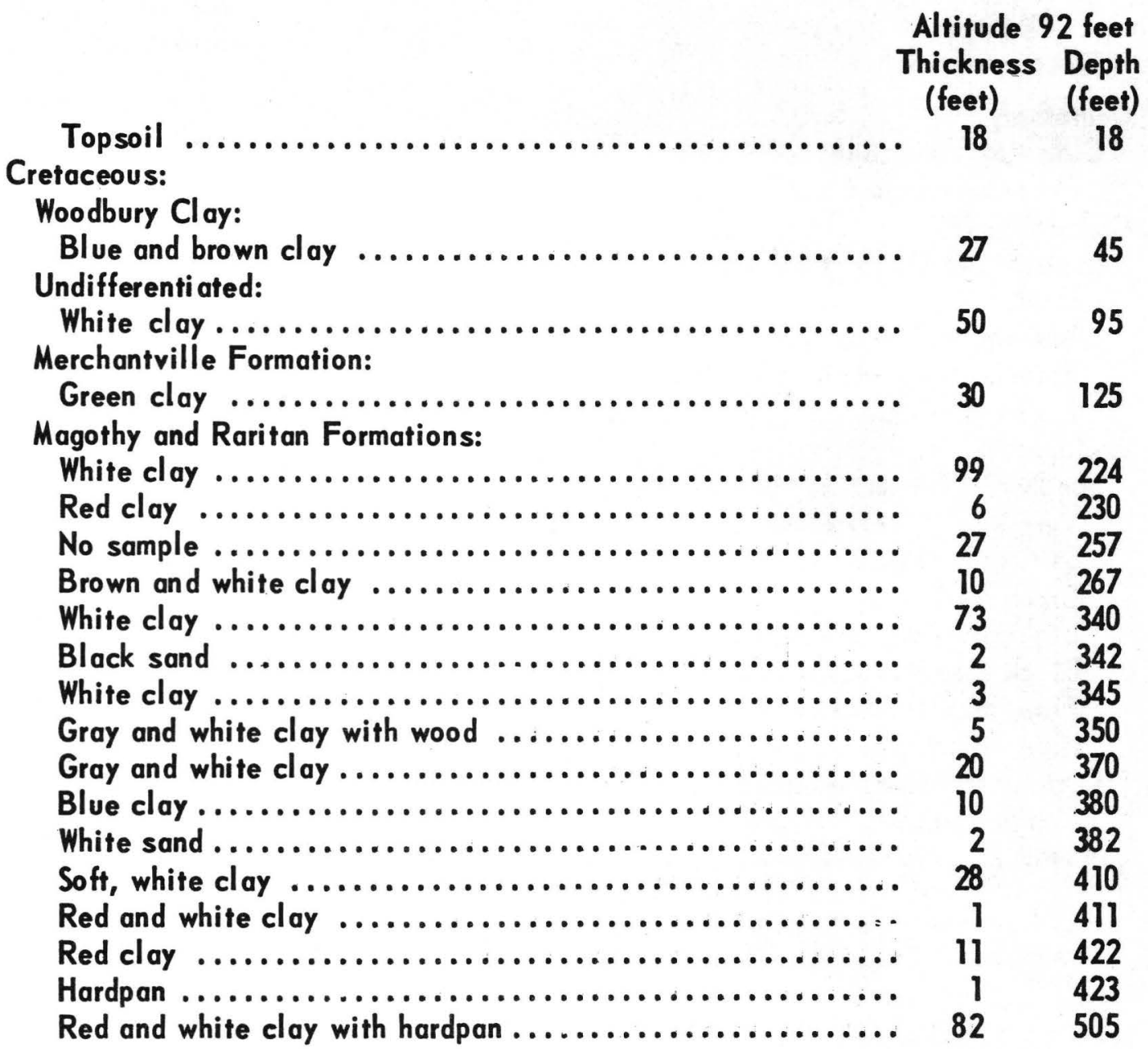


Table 3. - Selected drillers' logs of wells in Burlington County, N. J. - Continued Well no. 94, North Burlington Co. Regional Jr.-Sr. High School (Log by Russell D'Agostino)

Crefaceous:

Altitude 83 feet

Thickness Depth

(feet) (feet)

Marshalltown Formation:

Medium, brown sand and clay....................... $30 \quad 30$

Brown clay ................................. 55

Englishtown Formation:

Medium, brown sand ............................. $17 \quad 52$

Brown sand and. clay .......................... $11 \quad 63$

Undifferentiated:

Dark clay.................................... $92 \quad 155$

Gray gravel and clay .............................. 55

Dark clay ................................... $60 \quad 220$

Merchantville (?) Formation:

Dark clay with some stones ...................... $12 \quad 233$

Magothy and Raritan Formations:

Sand ........................................... $24 \quad 256$

Fine, brown sand ............................. 8264

Clay ........................................ $12 \quad 276$

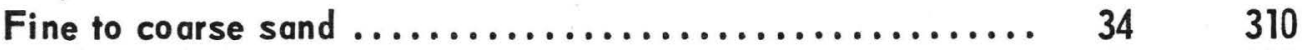

Sand and clay ................................. 10 320

Sand and clay with wood $\ldots \ldots \ldots \ldots \ldots \ldots \ldots \ldots . . . \ldots$ At 320

Sand and clay $\ldots \ldots \ldots \ldots \ldots \ldots \ldots \ldots \ldots \ldots \ldots \ldots \ldots \ldots \ldots \ldots, 16,336$

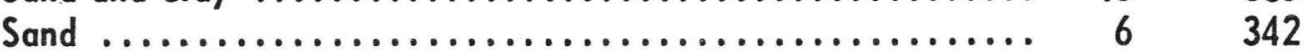

Dark clay .................................. 8350 
Table 3. - Selected drillers' logs of wells in Burlington County, N. J. - Continued Well no. 95, Ralph R. Parker

(Log by W. R. Clair)

\section{Cretaceous:}

Altitude 62 feet

Thickness Depth

(feet) (feet)

Englishtown Formation:

Clayey sand ................................. 15

Water-bearing sand $\ldots \ldots \ldots \ldots \ldots \ldots \ldots \ldots \ldots \ldots \ldots \ldots . . . \ldots$

Woodbury Clay and Merchantville Formation (undifferentiated):

Black clay ................................... 105

Water-bearing, green marl $\ldots \ldots \ldots \ldots \ldots \ldots \ldots \ldots \ldots \ldots \ldots$ 2

Undifferentiated:

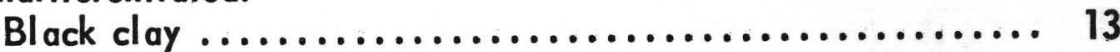

Magothy and Raritan Formations:

Fine to medium sand $\ldots \ldots \ldots \ldots \ldots \ldots \ldots \ldots \ldots \ldots \ldots \ldots, 5285$

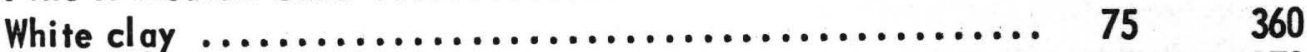

Fine sand $\ldots \ldots \ldots \ldots \ldots \ldots \ldots \ldots \ldots \ldots \ldots \ldots \ldots \ldots \ldots . \ldots \ldots$

Coarse sand ................................. $10 \quad 380$

Well no. 97, Peter Martin

(Log by John S. Wrobel)

Altitude 62 feet

Tertiary and Cretaceous:

Vincentown Formation, Hornerstown Sand, and Navesink

Formation (undifferentiated):

Marl

Cretaceous:

Mount Laurel Sand and Wenonah Formation:

Sandstone ................................. 20

Water-bearing, gray sand 
Table 3. - Selected drillers' logs of wells in Burlington County, N. J. - Continued Well no. 98, William E. Johnson Jr.

(Log by Charles L. Mollitor Inc.)

Topsoil

Tertiary:

Hornerstown Sand:

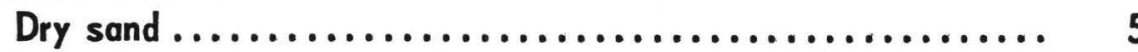

Water-bearing, green sand ........................ $36 \quad 43$

Cretaceous:

Navesink Formation:

Sandy marl $\ldots \ldots \ldots \ldots \ldots \ldots \ldots \ldots \ldots \ldots \ldots \ldots \ldots \ldots, \quad 32 \quad 75$

Mount Laurel Sand and Wenonah Formation:

Fine sand $\ldots \ldots \ldots \ldots \ldots \ldots \ldots \ldots \ldots \ldots \ldots \ldots \ldots, 50,125$

Marshall to wn Formation:

Black sandy marl: ............................. $70 \quad 195$

Englishtown Formation and Woodbury Clay (undifferentiated):

Green marl ...................................... $20 \quad 215$

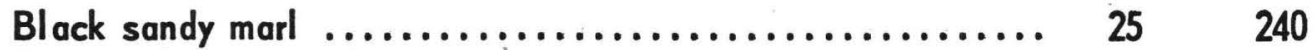

Gray marl ...................................... $5 \quad 245$

Hardpan and clay .............................. $10 \quad 255$

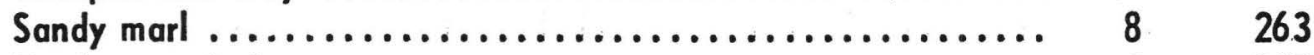

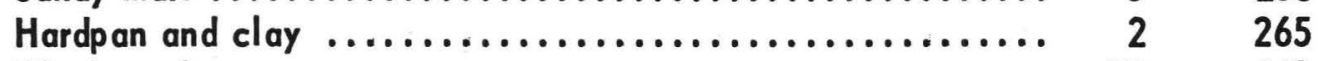

Black marl ................................. $73 \quad 338$

Merchantville Formation:

Green marl ..................................... $37 \quad 375$

Black marl ................................... $12 \quad 387$

Green marl .................................... $13 \quad 400$

Magothy and Raritan Formations:

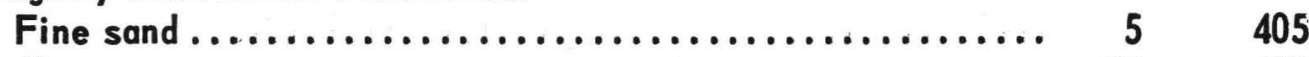

Clay ........................................ $15 \quad 420$

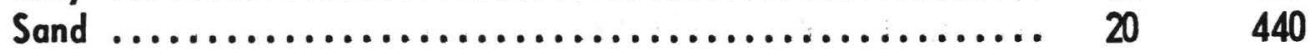


Table 3. - Selected drillers' logs of wells in Burlington County, N. J. - Continued Well no. 99, George Aaron

(Log by J. Henry Robbins)

Altitude 116 feet

Thickness Depth

(feet) (feet)

Tertiary:

Kirkwood Formation:

Yellow clay and fine sand $\ldots \ldots \ldots \ldots \ldots \ldots \ldots \ldots \ldots \ldots .60 . \ldots$

Tertiary and Cretaceous:

Manasquan and Vincentown Formations, Hornerstown Sand and

Navesink Formation (undifferentiated):

Black clay .............................. 70

150

Green clay .............................. $170 \quad 320$

Cretaceous:

Mount Laurel (?) Sand and Wenonah (?) Formation:

Green sand ................................... $20 \quad 340$

Well no. 100, William G. Freeman

(Log by J. Henry Robbins)

Tertiary:

Kirkwood Formation:

Yellow clay and sand

50

50

Tertiary and Cretaceous:

Manasquan and Vincentown Formations, Hornerstown Sand, and Navesink Formation (undifferentiated):

Black clay ................................... 100

Green clay ................................ $110 \quad 260$

Cretaceous:

Mount Laurel Sand and Wenonah Formation:

Green and white sand 
Table 3. - Selected drillers' logs of wells in Burlington County, N. J. - Continued

Well no. 102, Albert E. Crisp

(Log by Haines and Moore)

Quaternary:

Pensauken Formation:

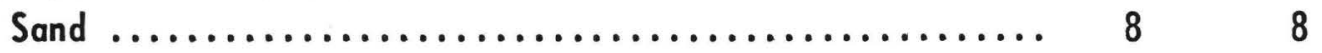

Cretaceous:

Marshalltown Formation:

Black clay ................................ $24 \quad 32$

Englishtown Formation, Woodbury $\mathrm{Cl}$ ay and Merchantville

Formation (undifferentiated):

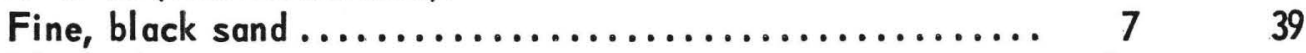

Black clay ................................. $86 \quad 125$

Sand, marl, and clay ............................ $24 \quad 149$

Black clay ................................ $28 \quad 177$

Magothy and Raritan Formations:

Sand

197

Well no. 104, William Haines, Jr.

(Log by Thomas C. Hagee, Jr.)

Qusaternary:

Altitude 52 feet

Cape May Formation:

Sand and loam .................................... 3

Sand and gravel ......................... 19

Cretaceous:

Marshalltown Formation:

Green marl ................................ 325

Englishtown Formation:

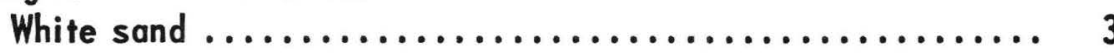


Table 3. - Selected drillers' logs of wells in Burlington County, N. J. - Continued Well no. 105, Roy W. Conrow

(Log by J. Henry Robbins)

Quaternary:

Cape May Formation:

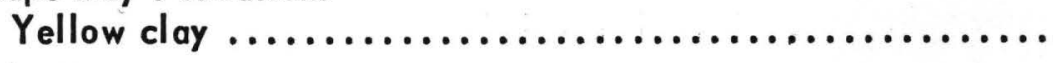

Altitude 37 feet

Thickness Depth

(feet)

(feet)

Cretaceous:

Englishtown Formation, Woodbury Clay, and Merchantville

Formation (undifferentiated):

Black clay .................................... 100

110

Green marl .................................. $80 \quad 190$

Magothy and Raritan Formations:

White clay and sand

Sand

$5 \quad 195$

......

10

205

Well no. 107, Thomas P. McGinnis

(Log by J. Henry Robbins)

Cretaceous:

Altitude 46 feet

Marshall town Formation:

Yellow clay and sand...

Black clay and sand

20

20

Englishtown Formation:

White sand ...

Woodbury Clay and Merchantville Formation (undifferentiated):

Black clay

140

250

Magothy and Raritan Formations:

Fine sand

White sand 
Table 3. - Selected drillers' logs of wells in Burlington County, N. J. - Continued

Well no. 108, Dominic Tuscano

(Log by Gus Hauser)

Cretaceous:

Englishtown Formation:

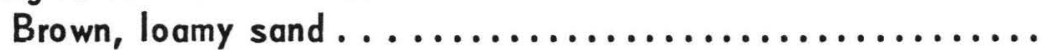

Green and brown sand

Altitude 28 feet

Thickness Depth

(feet) (feet)

Green and brown loamy sand ...................... $10 \quad 17$

Tan loamy sand $\ldots \ldots \ldots \ldots \ldots \ldots \ldots \ldots \ldots \ldots \ldots, \quad 7 \quad 24$

Englishtown Formation, Woodbury Clay, and Merchantville

Formation (undifferentiated):

Gray clay...................................... $24 \quad 48$

Black clay..................................... $24 \quad 72$

Brown and black clay ........................... $24 \quad 96$

Black clay .................................. $64 \quad 160$

Gray and black clay.......................... $30 \quad 190$

Magothy and Raritan Formations:

Fine, gray sand $\ldots \ldots \ldots \ldots \ldots \ldots \ldots \ldots \ldots \ldots \ldots . \ldots \ldots$ 2 2192

Fine, gray sand with some fine gravel $\ldots \ldots \ldots \ldots \ldots \ldots \ldots, 4 \quad 196$

Well no. 111, Eva Diamond

(Log by John Wrobel)

Cretaceous:

Navesink Formation, Mount Laurel Sand, and Wenonah Formation and Marshall town Formation (undifferentiated):
No sample..
Marl
100
$\mathrm{Clo}$
$20 \quad 120$
Mart
$12 \quad 132$
White sand with some clay $\ldots \ldots \ldots \ldots \ldots \ldots \ldots \ldots \ldots, \quad 7 \quad 150$
Marl ....................................... $6 \quad 156$

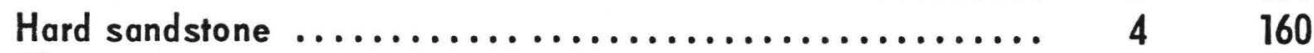
Clay ..................................... 19
Englishtown Formation:
Clay and black sand $\ldots \ldots \ldots \ldots \ldots \ldots \ldots \ldots \ldots \ldots \ldots, \quad 7 \quad 186$
Water-bearing, black sand with some shells ............ $16 \quad 202$ 
Table 3. - Selected drillers' logs of wells in Burlington County, N. J. - Continued Well no. 113, Samuel Dickter (Log by Charles L. Mollitor Inc.)

Altitude 50 feet Thickness Depth (feet) (feet)

\section{Cretaceous:}

Mount Laurel Sand and Wenonah Formation:

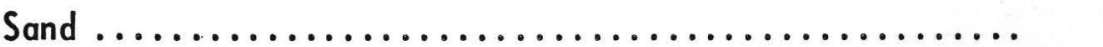

Black marl ................................ 38

$7 \quad 7$

Marshall town Formation:

Green sandy marl ............................ 80

Englishtown Formation:

Sand

$38 \quad 45$

Englishtown Formation, Woodbury Clay and Merchantville

Formation (undifferentiated):

Black marl ................................. 20

155

Green sand $\ldots \ldots \ldots \ldots \ldots \ldots \ldots \ldots \ldots \ldots \ldots \ldots \ldots, 5$

Black marl ................................... 50

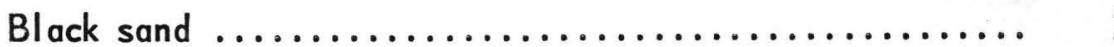

Green marl ..................................... 36

Hardpan ................................... 2

Green marl ..................................

Magothy and Raritan Formations:

Sand

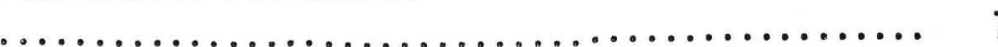

15300


Table 3. - Selected drillers' logs of wells in Burlington County, N. J. - Continued

Well no. 116, Charles J. Ruhle

(Log by James L. Ruhle and J. H. Robbins $4 /$ )

Quaternary:

Pensauken Formation:

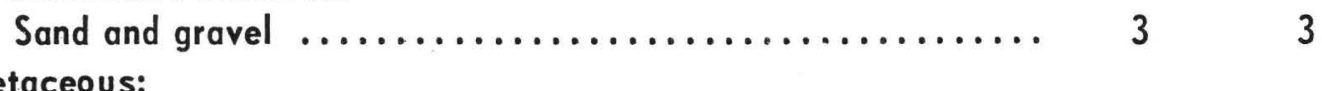

Cretaceous:

Mount Laurel Sand and Wenonah Formation:

Yellow sandy clay ......................... $17 \quad 20$

Marshall town Formation:

Glauconitic sandy clay ...................... $30 \quad 50$

Englishtown Formation:

Glauconitic sandy clay, but lighter in color ........... $80 \quad 130$

Woodbury $\mathrm{Cl}$ ay and Merchantville Formation (undifferentiated):

Dark clay with fossils ......................... $45 \quad 175$

Lighter-colored clay with fossils ................ $55 \quad 230$

Magothy and Raritan Formations:

Woiter-bearing sand

$10 \quad 240$

4/ Richards, 1954

Well no. 117, Felix Oliveto

(Log by Paul Steffens)

Altitude 63 feet

Topsoil

Quaternary:

Pensauken Formation:

Loam ...................................... 10

Gravel .................................... 2

12

$2 \quad 14$

\section{Cretaceous:}

Marshalltown Formation:

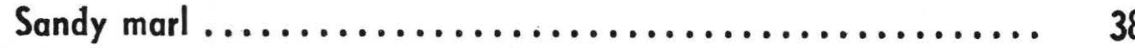

$38 \quad 52$

Englishtown Formation:

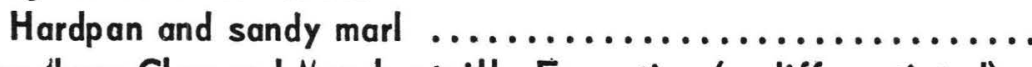

Woodbury $\mathrm{Cl}$ ay and Merchantville Formation (undifferentiated):

Marl

Magothy and Raritan Formations:

$130 \quad 217$

Sand

$6 \quad 223$ 
Table 3. - Selected drillers' logs of wells in Burlington County, N. J. - Continued Well no. 120, William Breur (Log by Charles L. Mollitor Inc.)

Topsoil Cretaceous:

Englishtown Formation:

Yellow sand

Altitude 78 feet

Thickness Depth

(feet) (feet)

Gravel

1

Wet sand

Green clay

Dry sand

Yellow sand

$\begin{array}{rr}8 & 9 \\ 4 & 13 \\ 9 & 22 \\ 7 & 29 \\ 3 & 32 \\ 46 & 78\end{array}$

Woodbury Clay:

Black mar

Gray clay

26

27

Sandy marl

7

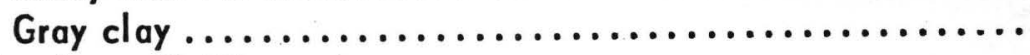

13

104

131

138

Merchantville Formation:

Black marl

$6 \quad 157$

Green marl

29

Black marl

28

186

Magothy and Raritan Formations:

White, sandy clay

$29 \quad 243$

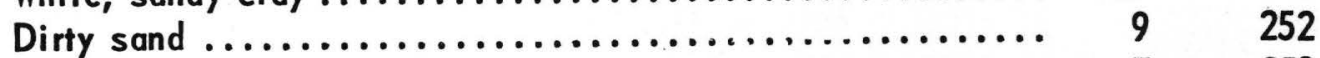

White sand $\ldots \ldots \ldots \ldots \ldots \ldots \ldots \ldots \ldots \ldots \ldots \ldots \ldots \ldots \ldots . \ldots \ldots$

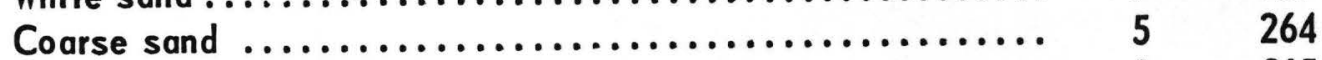

White clay ................................. 11265

Coarse, white sand $\ldots \ldots \ldots \ldots \ldots \ldots \ldots \ldots \ldots \ldots \ldots \ldots, 7272$ 
Table 3. - Selected drillers' logs of wells in Burlington County, N. J. - Continued

(Well no. 122, Joseph E. Rudderow)

(Log by Charles L. Mollitor Inc.)

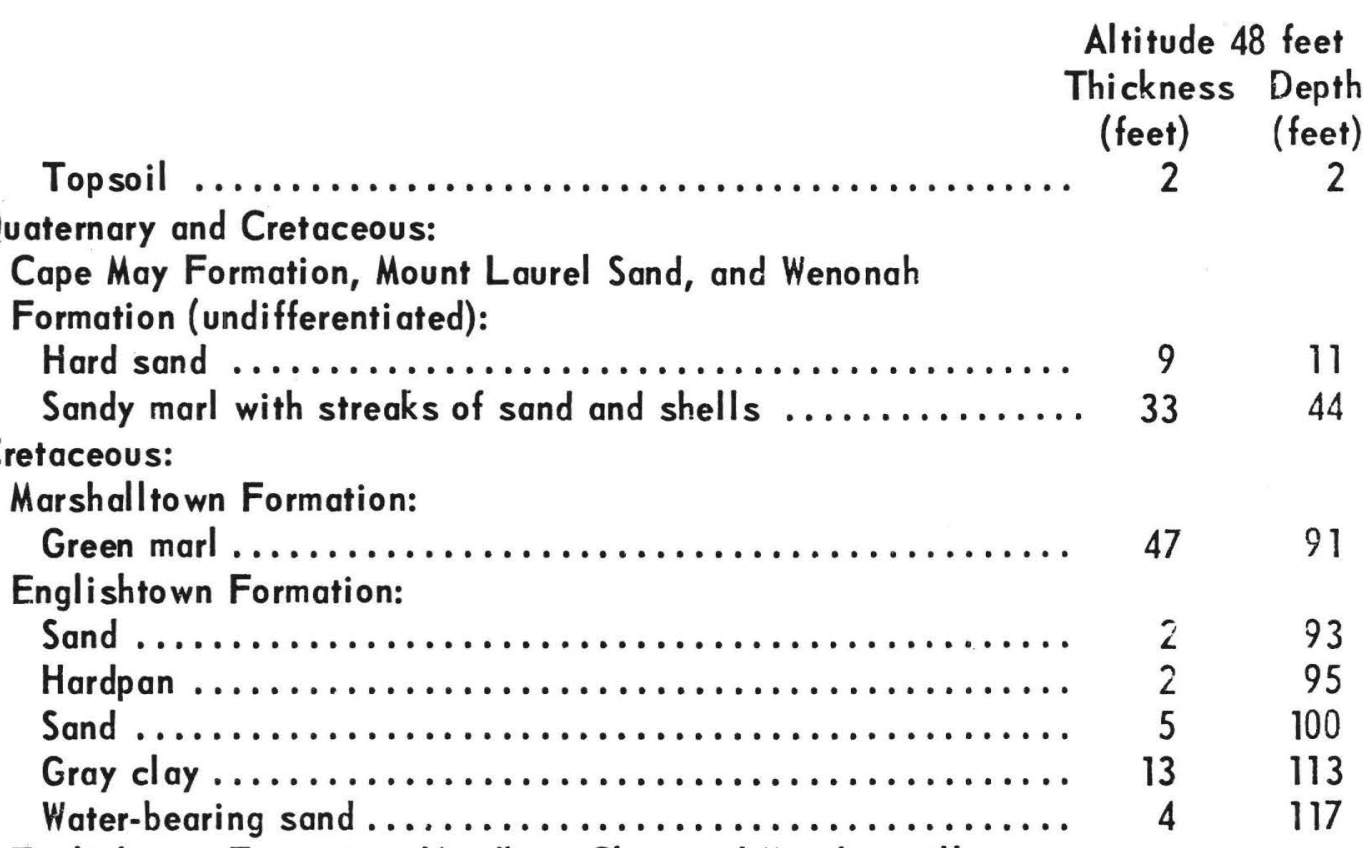

Englishtown Formation, Woodbury Clay, and Merchantville

Formation (undifferentiated):

No sample .............................. 121

238

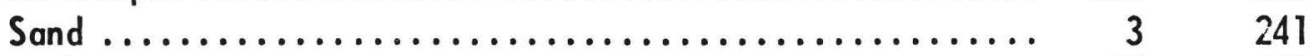

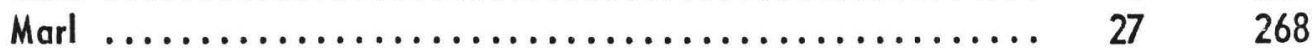

Magothy and Raritan Formations:

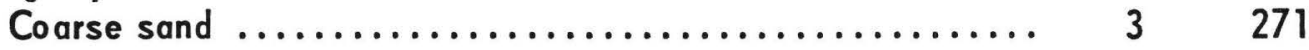

Red clay .................................... $10 \quad 281$

Sand .................................... 3284

Red and gray clay ......................... $43 \quad 327$

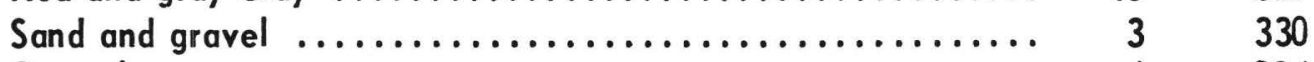

Gray clay ................................. 4334

Hardpan .................................... $25 \quad 359$

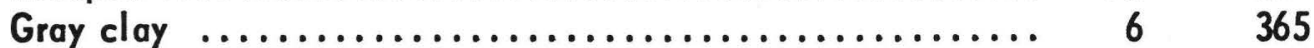

Hardpan .................................. $11 \quad 376$

Gray clay................................ $5 \quad 381$

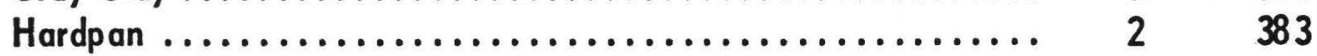

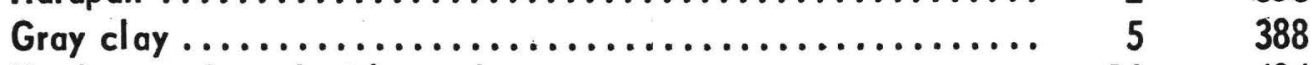

Hardpan and sand with wood $\ldots \ldots \ldots \ldots \ldots \ldots \ldots \ldots \ldots \ldots, 16 \ldots \ldots$

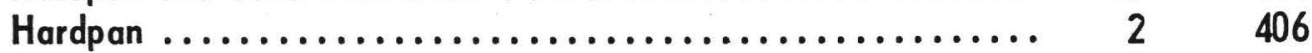

Streaks of sand and hardpan ...................... 7413

Red and gray clay ............................ $26 \quad 439$

Water-bearing, coarse sand with wood $\ldots \ldots \ldots \ldots \ldots \ldots \ldots . \ldots 17 \quad 456$

Gray clay ................................ 1 
Table 3. - Selected drillers' logs of wells in Burlington County, N. J. - Continued Well no. 126, Pittsburgh Plate Glass Co. (Log by Layne-New York Co.)

\begin{tabular}{|c|c|c|}
\hline & $\begin{array}{c}\text { Altitude } 6 \\
\text { Thickness } \\
\text { (feet) }\end{array}$ & $\begin{array}{l}5 \text { feet } \\
\text { Depth } \\
\text { (feet) }\end{array}$ \\
\hline \multicolumn{3}{|l|}{$\begin{array}{l}\text { Quaternary: } \\
\text { Pensauken Formation: }\end{array}$} \\
\hline Red sand ........... & 12 & 12 \\
\hline $\begin{array}{l}\text { Cretaceous: } \\
\text { V'oodbury Clay and Merchantville Formation (undifferentiated): }\end{array}$ & & \\
\hline $\begin{array}{l}\text { Blue clay } \ldots \ldots \ldots \ldots \ldots \ldots \ldots \ldots \ldots \ldots \ldots \ldots \ldots \ldots \ldots \ldots \ldots \ldots \ldots \ldots \ldots \ldots \\
\text { Magothy and Faritan Formations: }\end{array}$ & 126 & 138 \\
\hline 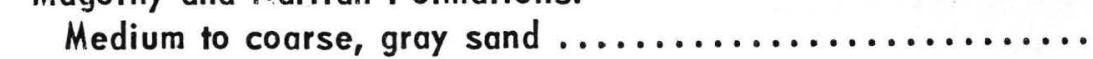 & 9 & 147 \\
\hline Tough, blue and gray clay ...... & 103 & 250 \\
\hline Coarse, gray sand and gravel ... & 33 & 283 \\
\hline 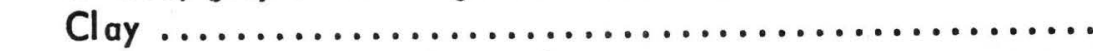 & 6 & 289 \\
\hline $\begin{array}{l}\text { Coarse, gray sand and gravel } \ldots \ldots \ldots \ldots \ldots \ldots \ldots \ldots \ldots \ldots \\
\text { Clay, coarse, gray sand, and gravel } \ldots \ldots \ldots \ldots \ldots \ldots \ldots \ldots \ldots\end{array}$ & $\begin{array}{r}26 \\
3\end{array}$ & $\begin{array}{l}315 \\
318\end{array}$ \\
\hline
\end{tabular}


Table 3. - Selected drillers' logs of wells in Burlington County, N. J. - Continued Well no. 128, Dunlap Carpet Works $5 /$

(Log by C. G. Orcutt)

Cretaceous:

Marshall town Formation:

Dark (marl) ................................ 43

Englishtown Formation:

Sand and gravel $\ldots \ldots \ldots \ldots \ldots \ldots \ldots \ldots \ldots \ldots \ldots \ldots \ldots, \quad 3 \quad 46$

Dark (marl) ................................. 61107

Fine sand ................................... $4 \quad 111$

Woodbury Clay and Merchantville Formation (undifferentiated):

Dark (marl) or clay .............................. $134 \quad 245$

Magothy and Raritan Formations:

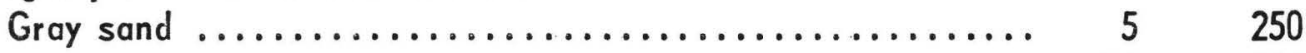

Dark sandy clay ............................. $12 \quad 262$

Red clay .................................. 11273

White clay .................................. 12274

Brown sandy clay ............................ $20 \quad 294$

White clay ................................. 2296

Light sandy clay $\ldots \ldots \ldots \ldots \ldots \ldots \ldots \ldots \ldots \ldots \ldots, 69 . \ldots \ldots$

Fed clay ................................... 2367

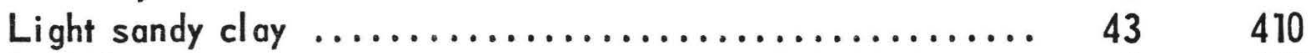

Red clay ..................................... $2 \quad 412$

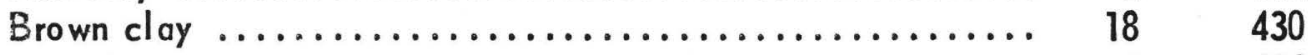

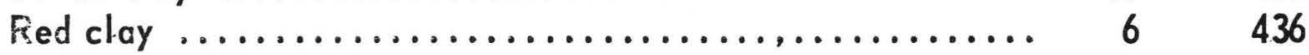

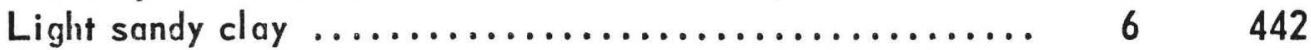

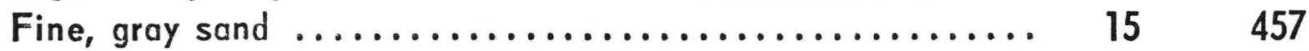

Sand and clay ............................... $52 \quad 509$

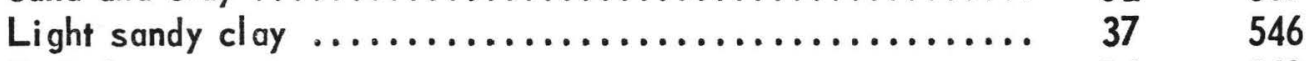

Red clay ................................... $14 \quad 560$

Sandy clay and fine sand $\ldots \ldots \ldots \ldots \ldots \ldots \ldots \ldots \ldots \ldots$. $48 \quad 608$

Yellow clay..................................... 396

Brown clay .................................. 9 9 920

Red clay $\ldots \ldots \ldots \ldots \ldots \ldots \ldots \ldots \ldots \ldots \ldots \ldots \ldots, 40.6 \%$

Fine sand with some brown clay .................... $7 \quad 667$

Fine and coarse sand and some gravel .............. $8 \quad 675$

5/ Smock, 1893, p. 303-30.4 
Table 3. - Selected drillers' logs of wells in Burlington County, N. J. - Continued

Well no. 129, Acme Market

(Log by Charles L. Mollitor Inc.)

Topsoil

Cretaceous:

Mount Laurel Sand and Wenonah Formation:

Yellow sand

Yellow clay

Marshall town Formation:

Gray marl

Soft marl

.............................

Brown sand

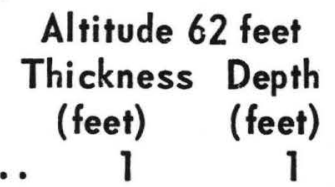

Black marl

Englishtown Formation:

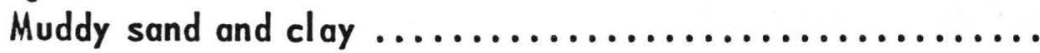

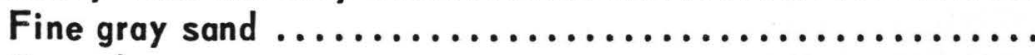

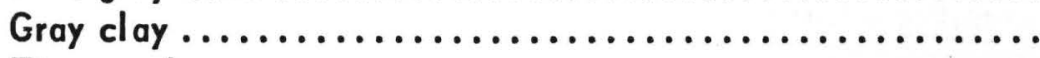

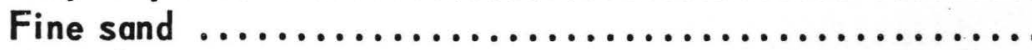

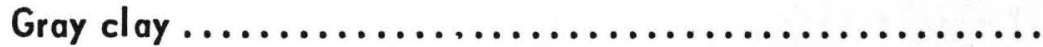

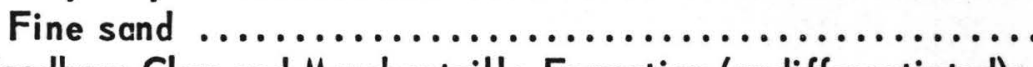

Woodbury Clay and Merchantville Formation (undifferentiated):

Gray clay ........................................

Green marl ......................................

Sticky, hard, black marl ..........................

$\begin{array}{rr}25 & 26 \\ 8 & 34 \\ 11 & 45 \\ 4 & 49 \\ 3 & 52 \\ 21 & 73 \\ 12 & 85 \\ 8 & 93 \\ 12 & 105 \\ 7 & 112 \\ 9 & 121 \\ 7 & 128\end{array}$

Magothy and Raritan Formations:

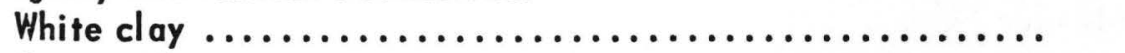

Gray marl .......................................

Hard, red clay ...................................

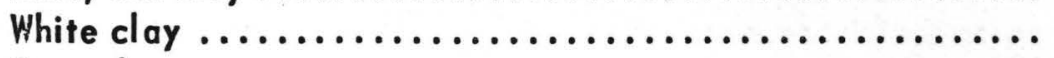

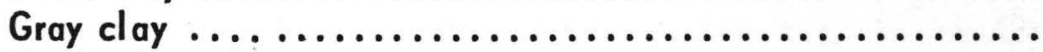

Hard, white clay ...................................

$80 \quad 208$

$35 \quad 243$

$35 \quad 278$

Sandy cl ay

2

280

$3 \quad 283$

$19 \quad 302$

$32 \quad 334$

$40 \quad 374$

$18 \quad 392$

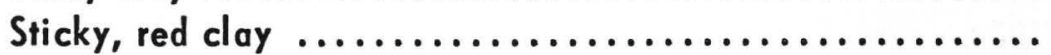

$21 \quad 413$

Gray clay

$8 \quad 421$

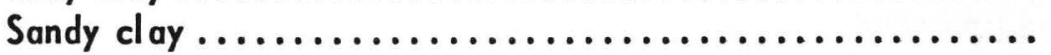

14

435

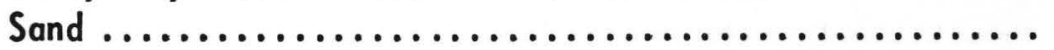

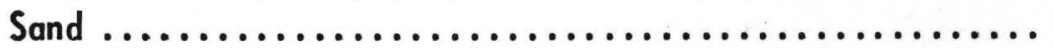

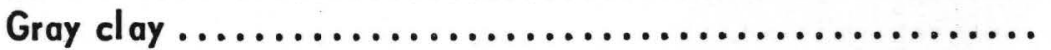

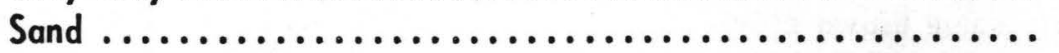

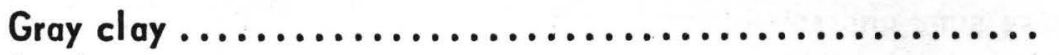

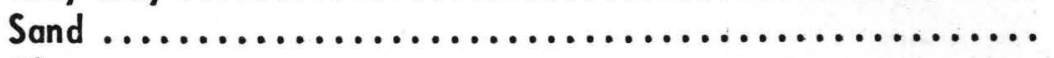

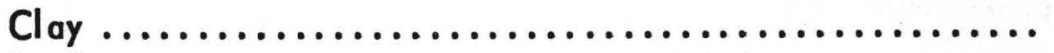

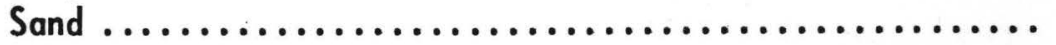

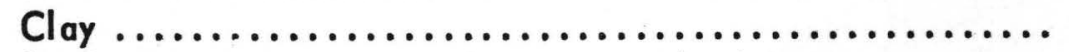

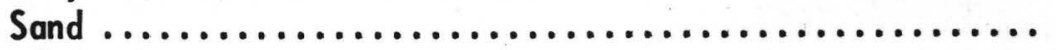

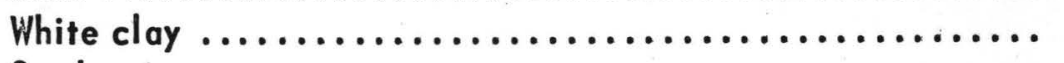

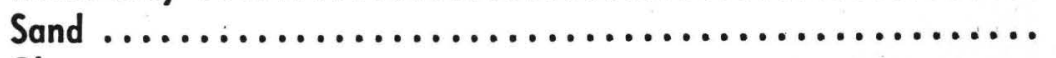

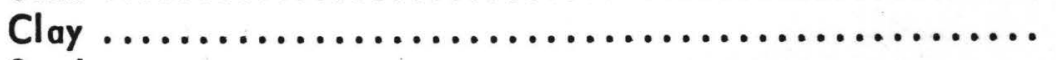

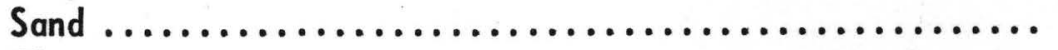

Clay 
Table 3. - Selected drillers' logs of wells in Burlington County, N. J. - Continued

Well no. 130, Jacobstown Baptist Church

(Log by Andrew White)

Quaternary, Tertiary, and Cretaceous:

Altitude 175 feet

Thickness Depth

(feet) (feet)

Pensauken, Kirkwood, and Vincentown Formations (undifferentiated):

Yellow sandy clay ............................ 48

48

Hornerstown and Red Bank Sands, and Navesink Formation

(undifferentiated):

Light-brown marl $\ldots \ldots \ldots \ldots \ldots \ldots \ldots \ldots \ldots \ldots \ldots \ldots, 17 \quad 65$

Dark-green marl ............................... $22 \quad 87$

Black clay and sand ............................ $13 \quad 100$

Gray clay and black sand with some shells ........... $20 \quad 120$

Cretaceous:

Mount Laurel Sand and Wenonah Formation:

Black sand with some fine gravel and shells ........... $8 \quad 128$

Gray to black sand with some fine gravel and shells....... $12 \quad 140$

Well no. 131, Jersey Central Power and Light Co. (Log by Greenhalgh and Kaye)

Tertiary:

Altitude 88 feet

Vincentown Formation:

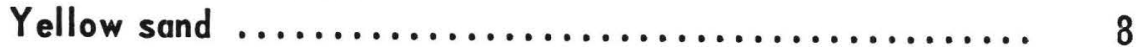

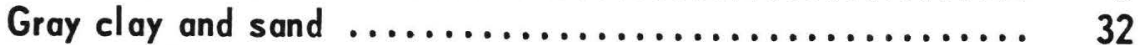

Tertiary and Cretaceous:

40

Hornerstown and Red Bank (?) Sands, and Navesink Formation (undifferentiated):

Dark-green and black marl ......................... $60 \quad 100$

Light-green marl ............................... $10 \quad 110$

Cretaceous:

Mount Laurel Sand and Wenonah Formation:

Sand with shells 
Table 3. - Selected drillers' logs of wells in Burlington County, N. J. - Continued Well no. 132, Edward H. Gancarz

(Log by Greenhalgh and Kaye)

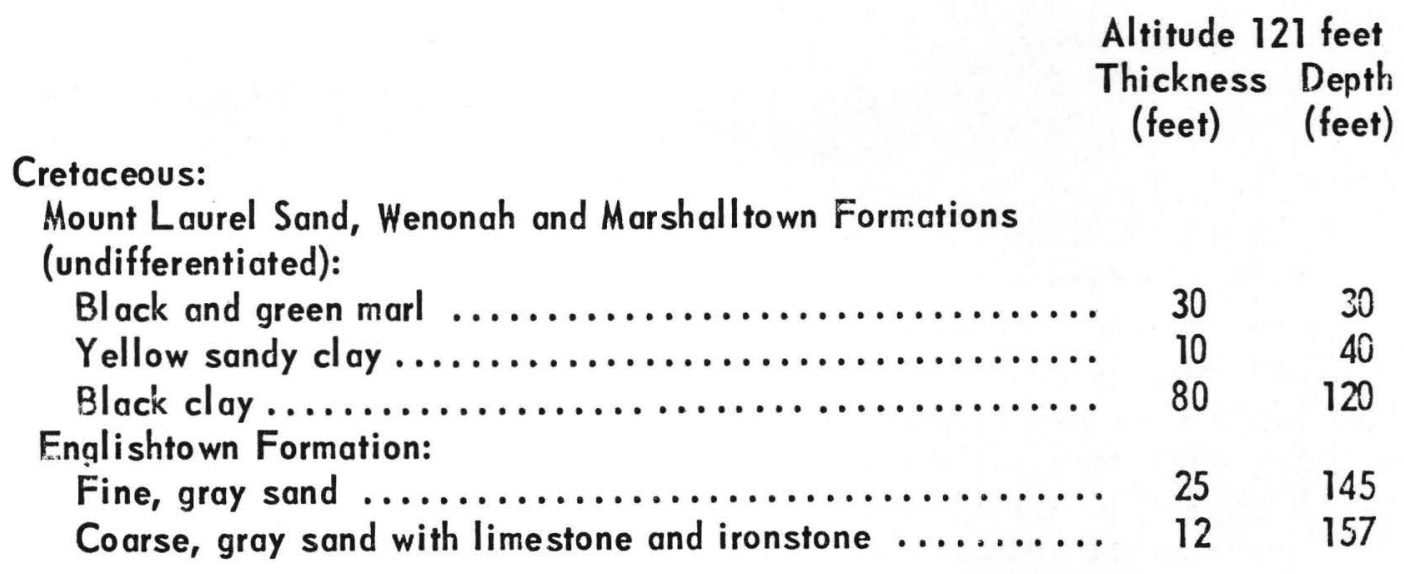

Well no. 134, Spartan Village, Inc.

(Log by Louis Bainbridge and Charles L. Mollitor Inc.)

Quaternary and Tertiary:

Altitude 154 feet

Bridgeton and Kirkwood Formations.(undifferentiated):

Tertiary:

Dry sand

25

25

Vincentown Formation:

Brown clay ................................ 328

Black marl................................... 1543

Black sandy marl ............................. $17 \quad 60$

Tertiary and Cretaceous:

Hornerstown Sand and Navesink Formation (undifferentiated):

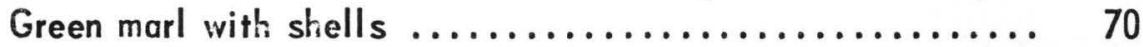

130

Cretaceous:

Mount Laurel Sand and Wienonah Formation:

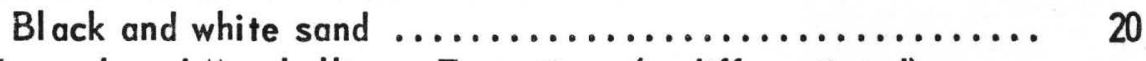

Wenonah and Marshalltown Formations (undifferentiated):

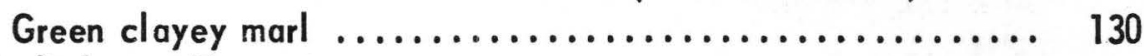

Englishtown Formation:

$\begin{array}{llll}\text { Brown clay with some pyrite, wood, and brown sand ........ } & 7 & 287\end{array}$

Hard, brown clay ............................. $18 \quad 305$

Englishtown Formation and Woodbury Clay (undifferentiated):

Hard, blue to brown clay ........................... 60

Hard, gray sand and sandy clay .................... 
Table 3. - Selected drillers' logs of wells in Burlington County, N. J. - Continued Well no. 136, B. F. Goodrich Co. (Log by Paul Steffens)

Quaternary:

Altitude 34 feet
Thickness
Depth
(feet) (feet)

Cape May Formation:

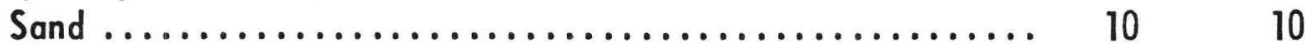

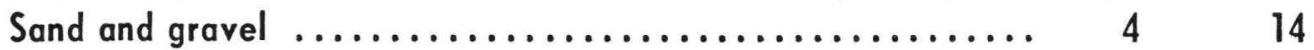

Cretaceous:

Magothy and Raritan Formations:

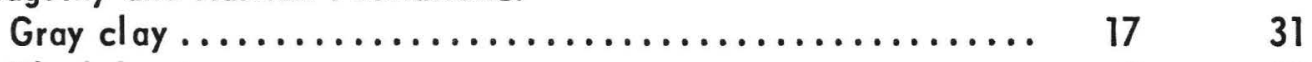

Black hardpan ............................... 23

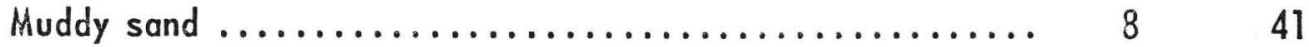

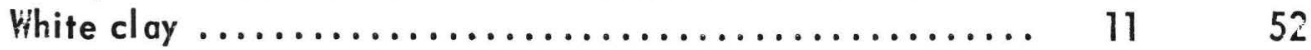

Muddy sand and gravel $\ldots \ldots \ldots \ldots \ldots \ldots \ldots \ldots \ldots \ldots, 6 \ldots \ldots$

White clay ................................. $17 \quad 75$

Sand and gravel............................... 3

Brown clay ................................. 19

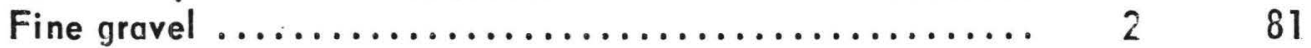

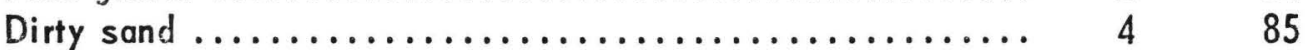

Dirty sand and gravel ............................ $21 \quad 106$

Brown sandy clay ........................... I 107

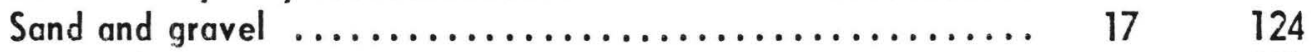

Sandy clay .................................. $5 \quad 129$

White clay ................................... 5 134

Sand and gravel ................................ 3 3137

White clay .................................... $4 \quad 141$

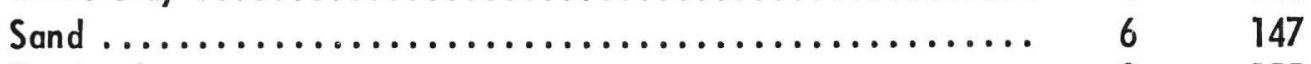

Sandy clay .................................. $8 \quad 155$

Reddish-brown clay ........................... 2157

Early Paleozoic (?):

Wissahickon Formation:

Soft rock ................................... 5162 
Table 3. - Selected drillers' logs of wells in Burlington County, N. J. - Continued

Well no. 138, Riverside-Alloy Metal Co.

(Log by Ridpath and Potter)

\section{Ouaternary and Cretaceous:}

Altitude 18 feet Thickness Depth

(feet) (feet)

Cape May, Magothy and Faritan Formations (undifferentiated):

Fine to coarse sand, clay, and some gravel ............ $120 \quad 120$ Early Paleozoic (?):

Wissahickon Formation:

Mica gneiss ................................ 680

800

Well no. 140, Jane Tabone

(Log by Charles L. Mollitor Inc.)

Altitude 130 feet

Ouaternary and Tertiary:

Bridgeton and Kirkwood Formations (undifferentiated):

Yellow sand and gravel ............................ $22 \quad 22$

Red sand and gravel $\ldots \ldots \ldots \ldots \ldots \ldots \ldots \ldots \ldots \ldots \ldots, 26 \ldots \ldots$

Tertiary:

Kirkwood Formation:

Yellow sand

22

70

Black mud ...

$5 \quad 75$

Muddy sand ...

$30 \quad 105$

Manasquan Formation:

Green marl $\ldots \ldots \ldots \ldots \ldots \ldots \ldots \ldots \ldots \ldots \ldots \ldots \ldots \ldots \ldots \ldots \ldots$
Vincentown Formation:

$25 \quad 130$

Green and black sand

10

140 
Table 3. - Selected drillers' logs of wells in Burlington County, N. J. - Continued

Well no. 141, A. F. Brunt

(Log by Charles L. Mollitor Inc.)

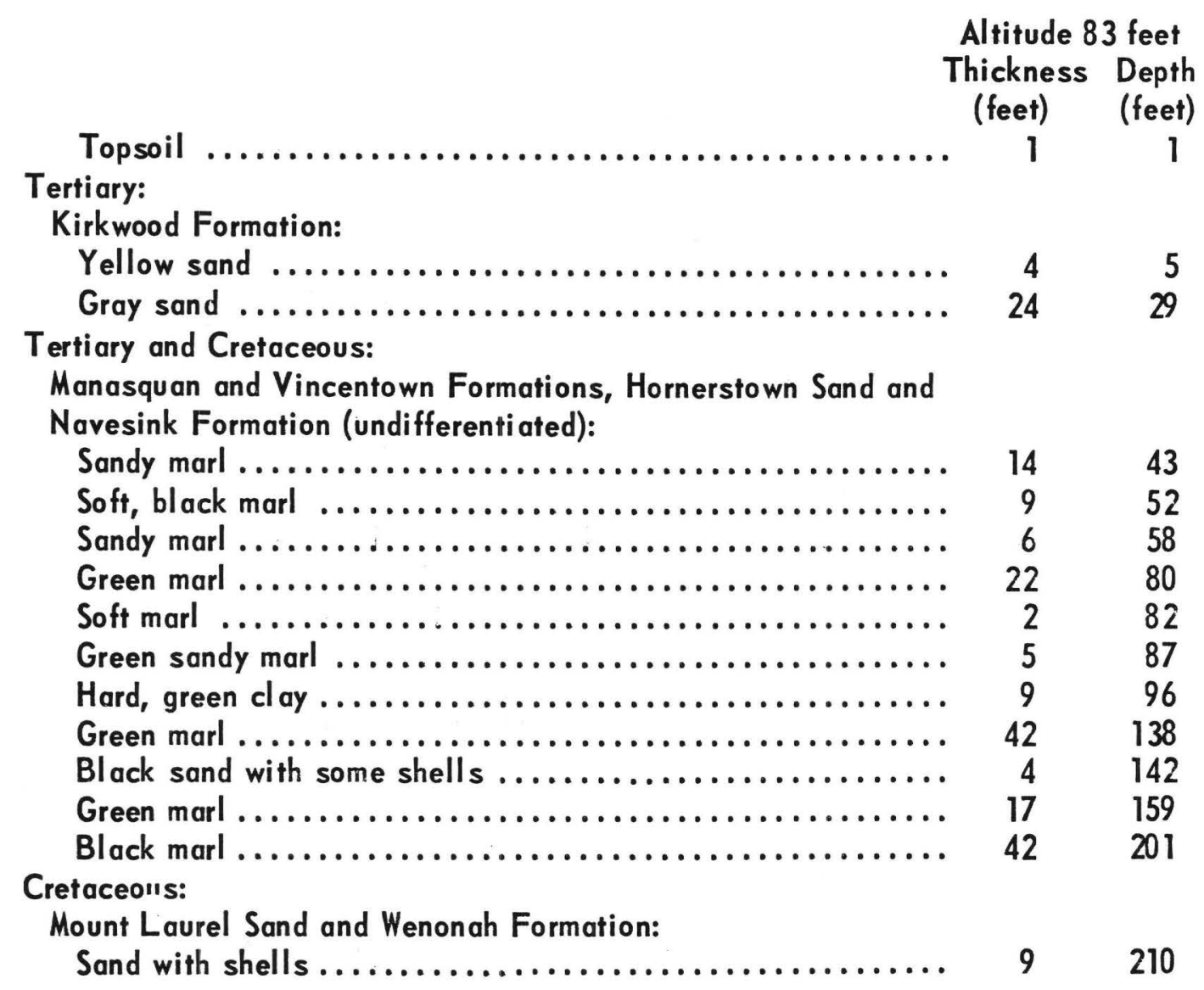


Table 3. - Selected drillers' logs of wells in Burlington County, N. J. - Continued

Well no. 143, Mr. Reilley

(Log by Henry B. Kummel 의)

Tertiary:

Kirkwood (?) Formation:

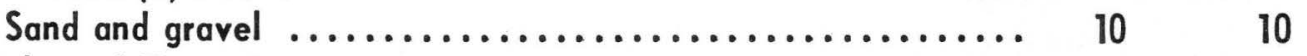

Kirkwood Formation:

Brown sandy clay ............................ $50 \quad 60$

Tertiary and Cretaceous:

Manasquan and Vincentown Formations, Hornerstown Sand and

Navesink Formation (undifferentiated):

Greenish clay with some marl and a trace of lignite and pebbles.................................... 80

Greensand (glauconite) marl .................... 80

Cretaceous:

Mount Laurel Sand and Wenonah Formation:

Fine, gray sand with some marl at the top and bottom ..... 110

Coarse, yellow sand with shells, clay, and very micaceous

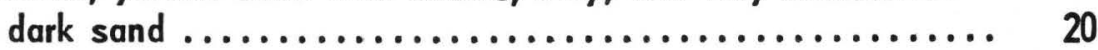

Marshall to wn Formation:

Black sandy clay ............................ 20

Very fine, greenish-black micaceous sand with glauconite .................................. 10

No sample .................................. 10

Englishtown Formation:

Fine, gray quartz sand $\ldots \ldots \ldots \ldots \ldots \ldots \ldots \ldots \ldots, 10$

Fine quartz sand with some glauconite $\ldots \ldots \ldots \ldots \ldots \ldots . .20$

Water-bearing, coarse quartz sand .................. 10

6) Kummel, 1910, p. $85-90$ 
Table 3. - Selected drillers' logs of wells in Burlington County, N. J. - Continued

Well no. 144, Joseph O'Neill

(Log by A. C. Schiultes and Sons)

Tertiary:

Kirkwood Formation:

Sand and gravel ............................. $60 \quad 60$

Yellow sandy clay ............................ $20 \quad 80$

Manasquan Formation:

Green marl ..................................... $38 \quad 118$

Tertiary and Cretaceous:

Vincentown Formation, Hornersto wan Sand, and Navesink

Formation (undifferentiated):

Hard sand ................................... 2120

Green clay.................................. $25 \quad 145$

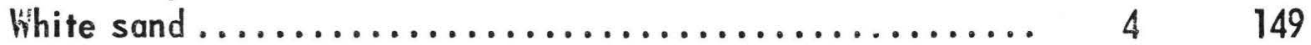

Clay and green marl $\ldots \ldots \ldots \ldots \ldots \ldots \ldots \ldots \ldots \ldots \ldots$. $26 \quad 175$

Green marl ...................................... $6 \quad 181$

Clay and green marl ........................... $52 \quad 233$

Green marl and sand with shells ..................... 27260

Green marl ..................................... $15 \quad 275$

Green marl with some sand ...................... $16 \quad 291$

Cretaceous:

Miount Laurel Sand and Henonah Formation:

Sand and gravel ............................... 9 9300

Clayey sand .................................... 19 319

Medium and coarse sand ......................... 18 337 
Table 3. - Selected drillers' logs of wells in Burlington County, N. J. - Continued Well no. 145, Country Lakes Inc. (Log by Charles L. Mollitor Inc.)

Tertiary:

$\begin{array}{cc}\text { Altitude } 82 \text { feet } \\ \text { Thickness } & \begin{array}{c}\text { Depth } \\ \text { (feet) }\end{array}\end{array}$

Kirkwood Formation:

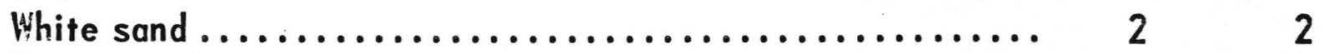

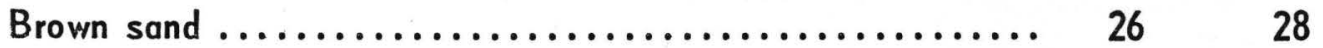

Sandy marl ............................... $23 \quad 51$

Fine, dirty sand ............................ $11 \quad 62$

Sandy marl ................................ 7

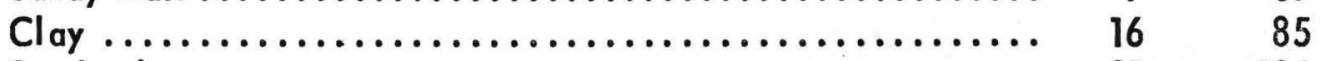

Sandy clay ................................... $21 \quad 106$

Tertiary and Cretaceous:

Manasquan and Vincentown Formations, Hornerstown Sand, and Navesink Formation (undifferentiated):

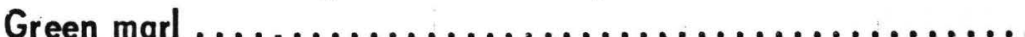

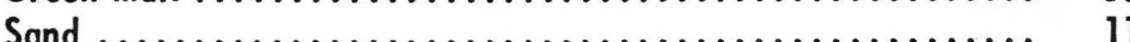

$63 \quad 169$

Sticky, green marl ......................... $80 \quad 260$

Green marl with shells ....................... $61 \quad 321$

Cretaceous:

Mount Laurel (?) Sand and Wenonah (?) Formation:

Gray sandy clay ............................ $8 \quad 329$

Mount Laurel Sand and Wenonah Formation:

Sand with shells ............................ $16 \quad 345$ 
Table 3. - Selected drillers' logs of wells in Burlington County, N. J. - Continued

Well no. 148, Helis Stock Farm

Cretaceous: (Log by William Blaisdell and Orcutt Bros. 그)

Mount Laurel Sand and Wenonah Formation:

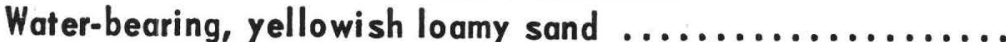

Fine sand with dark mud.

Marshall town Formation:

Stiff, black sandy clay

Water-bearing, fine, muddy

Stiff, black sandy clay

Englishtown Formation:

Water-bearing, fine sand with scattered layers of sandstone, clay, and shells

Black sandy clay

Water-bearing, fine sand

Black sandy clay

Water-bearing, fine sand

Woodbury Clay and Merchantville Formation (undifferentiated):

Dark sandy clay with scattered layers of sandstone and shells

Dark sandy clay and scattered layers of sandstone, shells, and wood

Magothy and Raritan Formations:

Fine sand with some gravel and brown clay

Red and white clay

Sand and sandstone with considerable wood and some clay

No sample

Fine sand .

Coarse sand which yields some water

Coarse gravel

Fine sand

White clay.

White sand and some coarse gravel ..................

Fine, white sand

Dark micaceous sand

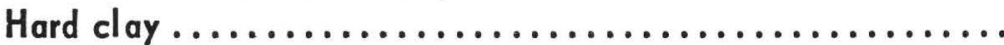

Coarse, dark sand and red clay ....................

Fine, white sand $\ldots \ldots \ldots \ldots \ldots \ldots \ldots \ldots \ldots \ldots \ldots$

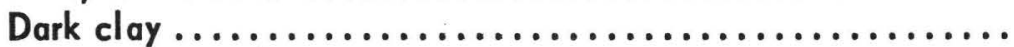

White and red $\mathrm{cl}$ ay $\ldots \ldots \ldots \ldots \ldots \ldots \ldots \ldots \ldots \ldots$

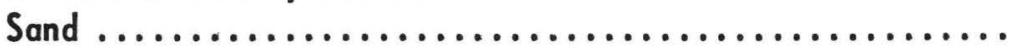

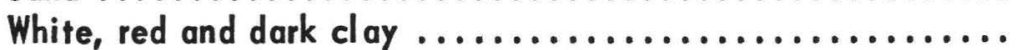

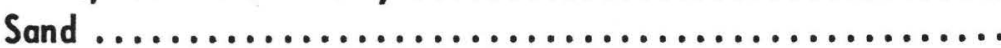

Coarse, white sand with clay $\ldots \ldots \ldots \ldots \ldots \ldots \ldots \ldots \ldots$

Coarse sand and gravel ..........................

Fine sand $\ldots \ldots \ldots \ldots \ldots \ldots \ldots \ldots \ldots \ldots \ldots \ldots \ldots \ldots \ldots \ldots \ldots \ldots$

Clay ....................................

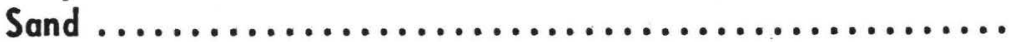

White clay $\ldots \ldots \ldots \ldots \ldots \ldots \ldots \ldots \ldots \ldots \ldots \ldots \ldots \ldots$

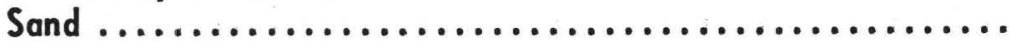

White clay $\ldots \ldots \ldots \ldots \ldots \ldots \ldots \ldots \ldots \ldots \ldots \ldots \ldots \ldots \ldots \ldots$

Gravel and coarse sand ........................

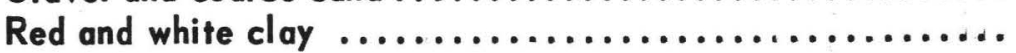

Sand
Altitude approx. 70 feet

Thickness Depth

(feet) (feet)

$14 \quad 14$

$34 \quad 48$

$24 \quad 72$

173

$9 \quad 82$

$34 \quad 116$

$1 \quad 117$

$7 \quad 124$

$1 \quad 125$

$3 \quad 128$

$50 \quad 178$

$128 \quad 306$

$8 \quad 314$

$24 \quad 338$

$18 \quad 356$

$11 \quad 367$

$3 \quad 370$

$17 \quad 387$

3390

$5 \quad 395$

$5 \quad 400$

$10 \quad 410$

$22 \quad 432$

$8 \quad 440$

$11 \quad 451$

$8 \quad 459$

$20 \quad 479$

$4 \quad 483$

$12 \quad 495$

2497

$78 \quad 575$

$13 \quad 588$

$12 \quad 600$

3603

$16 \quad 619$

$25 \quad 644$

$7 \quad 651$

$13 \quad 664$

$15 \quad 679$

$2 \quad 681$

$9 \quad 690$

$25 \quad 715$

At $\quad 715$ 
Table 3. - Selected drillers' logs of wells in Burlington County, N. J. - Continued

Well no. 149, Kauffman and Minteer Inc.

(Log by Charles L. Mollitor Inc.)

\begin{tabular}{|c|c|c|}
\hline & $\begin{array}{c}\text { Altitude } 7 \\
\text { Thickness } \\
\text { (feet) }\end{array}$ & $\begin{array}{l}4 \text { feet } \\
\text { Depth } \\
\text { (feet) }\end{array}$ \\
\hline Fill $\ldots \ldots \ldots \ldots \ldots \ldots \ldots \ldots \ldots \ldots \ldots \ldots \ldots \ldots \ldots \ldots$ & 1 & 1 \\
\hline 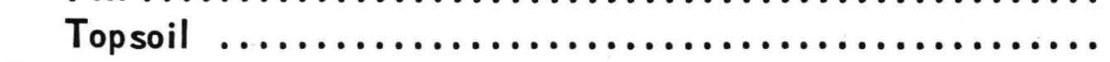 & 1 & 2 \\
\hline $\begin{array}{l}\text { Cretaceous: } \\
\text { Mount Laurel Sand and Wenonah Formation: }\end{array}$ & & \\
\hline 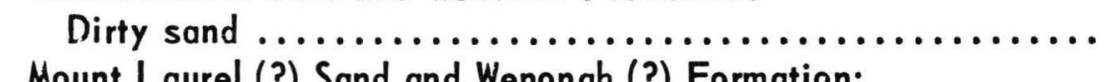 & 17 & 19 \\
\hline $\begin{array}{l}\text { Sandy marl } \ldots \ldots \ldots \ldots \ldots \ldots \ldots \ldots \ldots \ldots \ldots \ldots \ldots \ldots \ldots \ldots \ldots \ldots \\
\text { Marshall town Formation: }\end{array}$ & 39 & 58 \\
\hline $\begin{array}{l}\text { Marl } \ldots \ldots \ldots \ldots \ldots \ldots \ldots \ldots \ldots \ldots \ldots \ldots \ldots \ldots \ldots \ldots \\
\text { Englishtown Formarion: }\end{array}$ & 36 & 94 \\
\hline 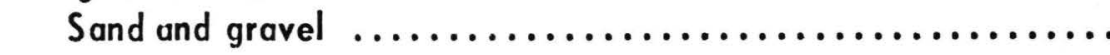 & 13 & 107 \\
\hline
\end{tabular}


Table 3. - Selected drillers' logs of wells in Burlington County, N. J. - Continued

Well no. 151, Helis Stock Farm

(Log by Ridpath and Potter)

Tertiary and Cretaceous:

Altitude 96 feet

Thickness Depth

(feet) (feet)

Undifferentiated:

Green sandy marl with some cemented sand ........... $342 \quad 342$

Soft, dark clay ............................ $69 \quad 411$

Cretaceous:

Magothy and Raritan Formations:

Hardpan with layers of light-colored sand ............. $29 \quad 440$

Hardpan with wood ......................... $18 \quad 458$

Hardpan with some thin streaks of sand ............ 40498

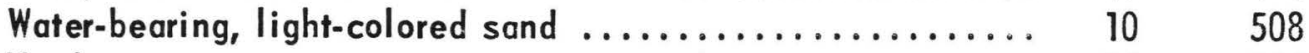

Hardpan ................................ 14

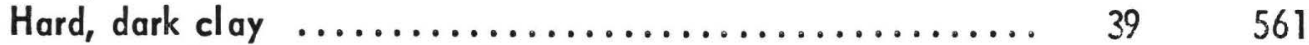

Very fine, light-colored sand ................... i2 579

Tough, dark clay .......................... $4 \quad 583$

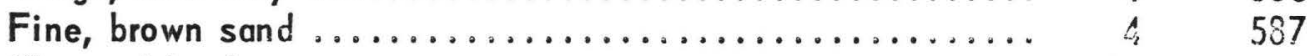

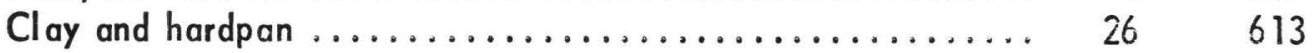

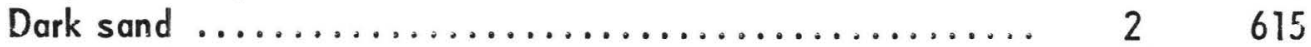

Very hard, white clay .......................... 5 620

Hard, red clay ........................... $87 \quad 707$

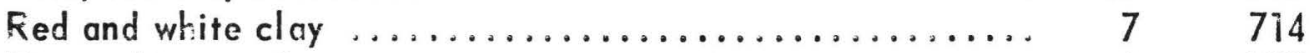

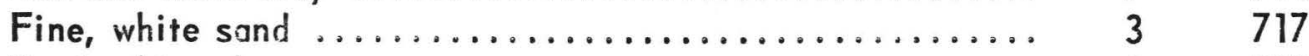

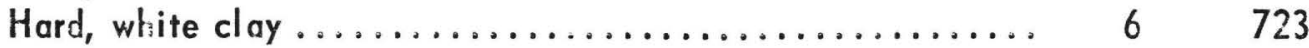

Hardpan ................................. 21

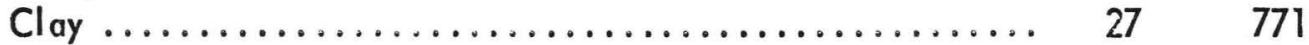

Hardpan ................................ $11 \quad 782$

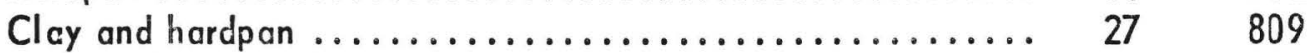

Light-colored sand ............................ $5 \quad 814$

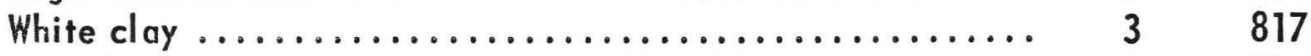

Red clay ............................... $78 \quad 895$

Sand ..................................... 1896

Hardpan ............................... 7903

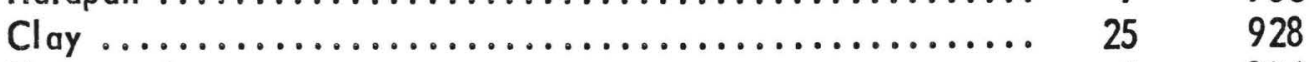

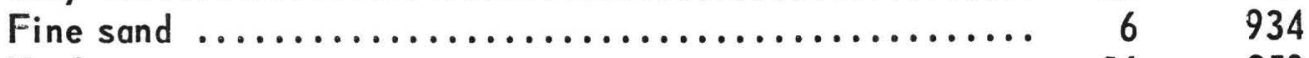

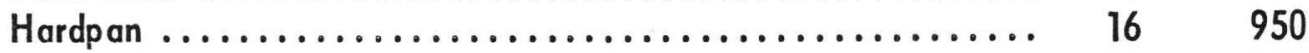

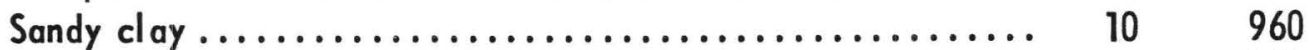

Hardpan ................................... 3963

No sample ................................ $24 \quad 987$

Early Paleozoic (?):

Wissahickon (?) Formation:

Hard sandstone, probably weathered bedrock ............ 1988 
Table 3. - Selected drillers' logs of wells in Burlington County, N. J. - Continued Well no. 152, Ridge Stables (Log by Paul Steffens)

Altitude 41 feet Thickness Depth

(feet) (feet)

Civaternary:

Cape Hay Formation:

Englishtown Formation:

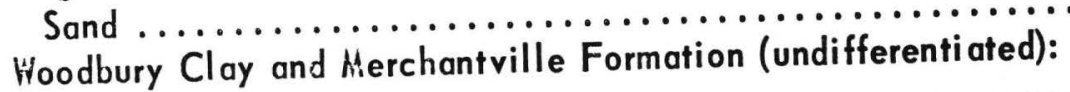
Marl.

165 216

Magothy and Raritan Formations:

Sand witl: wood and streaks of clay

Well no. 155, Tallman Brothers, Inc.

(Log by W. R. Clair)

Altitude 42 feet

Quaternary:

Cape May Formation:

Fine sand

18

18

Yellow clay

6

24

\section{Cretaceous:}

Englishtown Formation:

Fine sand 
Table 3. - Selected drillers' logs of wells in Burlington County, N. J. - Continued Well no. 157, Ross Monroe (Log by Harvey Beebe Sr.)

Yellow topsoil Altitude 46 feet Thickness Depth

(feet) (feet) Quaternary:

Cape May Formation:

Gravel

Tertiary:

Kirkwood Formation:

Black clay

Tertiary and Cretaceous:

Manasquan and Vincentown Formations, Hornerstown Sand, and Navesink Formation (undifferentiated):

Marl

Cretaceous:

Mount Laurel Sand and Wenonah Formation:

Black and white sand

80

237

Marshall town Formation:

Hardpan

Well no. 158, Hampton Lakes Development Co. (Log by Charles L. Mollitor Inc.)

Altitude 49 feet

Loamy topsoil

Quaternary:

Cape May Formation:

Sand

8

Terti ary:

Kirkwood Formation:

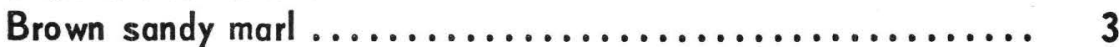

Black marl .................................. 13

Manasquan and Vincentown Formations and Hornerstown Sand

(undifferentiated):

Light-green marl ................................. $83 \quad 142$

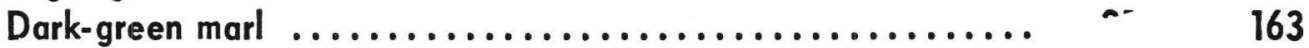

\section{Cretaceous:}

Navesink Formation:

Black marl with shells ............................. $6 \quad 169$

Black marl .................................. $29 \quad 198$

Navesink (?) Formation:

Sandy marl with sand streaks ..................... $47 \quad 245$

Mount Laurel Sand and Wenonah Formation:

Sand with shells and clay streaks. 
Table 3. - Selected drillers' logs of wells in Burlington County, N. J. - Continued Well no. 159, Vincentown Diner

(Log by Edward Robbins Sr.)

Tertiary and Cretaceous:

Altitude 72 feet Thickness Depth (feet) (feet)

Hornerstown Sand and Navesink Formation (undifferentiated):

Black clay

\section{Cretaceous:}

Mount Laurel Sand and Wenonah Formation:

Sand with iron oxide stains

Marshall town Formation:

Black clay

Englishtown Formation:

Sand

Tertiary:

Cohan sey Sand:

Yellow sand and gravel ...................... 20

Altitude 140 feet

Clay and gravel

Black clay................................ 15

Gravel .................................. 10

Clay .....................................

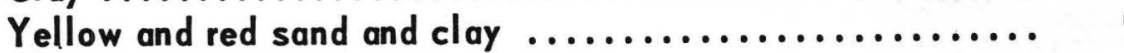

$9 \quad 69$


Table 3. - Selected drillers' logs of wells in Burlington County, N. J. - Continued

Well no. 162, A. Marone

(Log by Charles L. Mollitor Inc.)

Topsoil

Altitude 40 feet

Thickness Depth

(feet) (feet)

Cretaceous:

Englishtown Formation:

Yellow sand ...................................... 14

Englishtown (?) Formation:

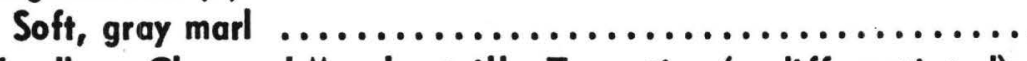

Woodbury Clay and Merchantville Formation (undifferentiated):

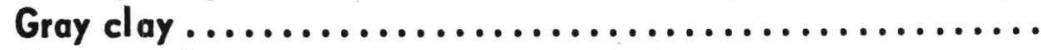

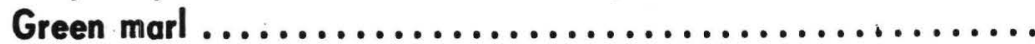

Magothy (?) and Raritan (?) Formation:

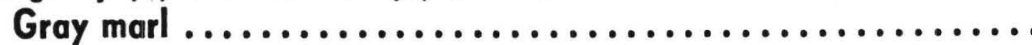

Magothy and Raritan Formation:

Sand...

Well no. 165, Alexander Construction Co. (Log by Paul Steffens)

Altitude 57 feet

Loamy top soil

Cretaceous:

Englishtown Formation:

Dry sand

Water-bearing sand and gravel .

Sandy marl

Brown sand

Woodbury $\mathrm{Cl}$ ay and Merchantville Formation (undifferentiated):

Marl

Magothy and Raritan Formations:

Dark-gray clay

Coarse sand and gravel

$13 \quad 215$


Table 3. - Selected drillers' logs of wells in Burlington County, N. J. - Continued Well no. 166, John S. Pew

(Log by Charles L. Mollitor Inc.)

Topsoil

Englishtown Formation:

Yellow sand

Woodbury (?) Clay:

Gray marl .................................

Woodbury Clay and Merchantville Formation (undifferentiated):

Hard, black marl ...........................

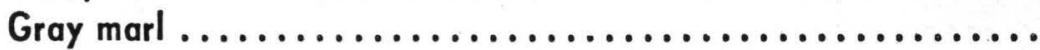

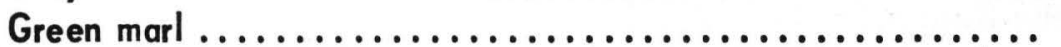

Soft, gray marl $\ldots \ldots \ldots \ldots \ldots \ldots \ldots \ldots \ldots \ldots \ldots \ldots \ldots$

Green marl

154

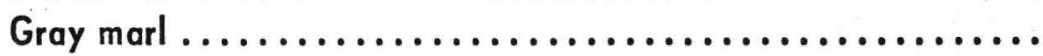

Magothy and Raritan Formations:

Sand

163

$48 \quad 211$

17

228

Well no. 169, John W. Hampton

(Log by Charles L. Mollitor Inc.)

Altifude 55 feet

Top soil

Cretaceous:

Mount Laurel Sand and Wenonah Formation:

Sand

Marshall town Formation:

Marl ................................... 93

128

Hardpan ................................ 2

130

Englishtown Formation:

Muddy sand

Sand 
Table 3. - Selected drillers' logs of wells in Burlington County, N. J. - Continued

Well no. 171, Ionac Chemical Co.

(Log by A. C. Schultes and Sons)

Quaternary:

$\begin{array}{cc}\text { Altitude } 30 \text { feet } \\ \text { Thickness } & \begin{array}{c}\text { Depth } \\ \text { (feet) }\end{array} \\ \text { (feet) }\end{array}$

Cape May Formation:

Fine to coarse, brown sand and gravel

Tertiary and Cretaceous:

Hornerstown Sand and Navesink Formation (undifferentiated):

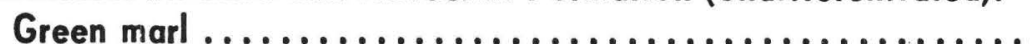

Dark-gray clay ................................ 15

$40 \quad 50$

Cretaceous:

Mount Laurel Sand and Wenonah Formation:

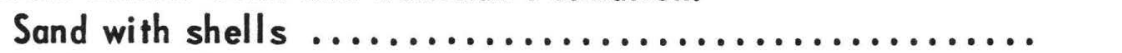

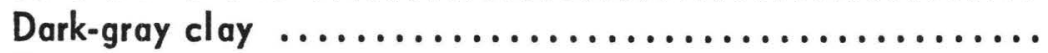

Fine to coarse, green sand $\ldots \ldots \ldots \ldots \ldots \ldots \ldots \ldots \ldots$

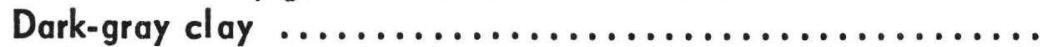

Fine to medium, gray sand $\ldots \ldots \ldots \ldots \ldots \ldots \ldots \ldots \ldots$

Dark-gray silty clay

Fine, greenish-gray sand

Marshalltown Formation:

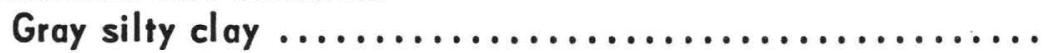

Dark-gray clay

$7 \quad 72$

$5 \quad 77$

1592

$11 \quad 103$

$33 \quad 136$

$12 \quad 148$

$7 \quad 155$

Englishtown Formation:

Fine, gray sand.$\ldots \ldots \ldots \ldots \ldots \ldots \ldots \ldots \ldots \ldots \ldots \ldots$

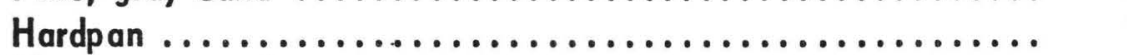

Fine, gray sand $\ldots \ldots \ldots \ldots \ldots \ldots \ldots \ldots \ldots \ldots \ldots \ldots \ldots \ldots \ldots$

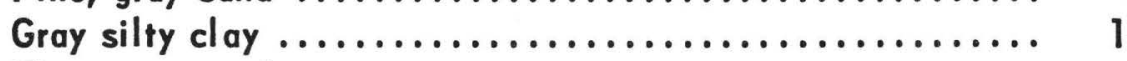

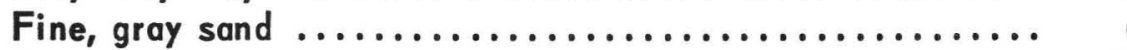

Gray, silty clay ........................... 19

$37 \quad 192$

11203

Woodbury Clay:

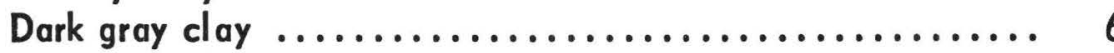

Gray silty clay ............................. 34

$65 \quad 322$

3435

Merchantville Formation:

Hardpan ................................ 2358

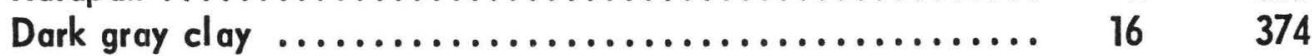

Hardpan .................................. $15 \quad 389$

Dark gray clay .............................. $25 \quad 414$

Magothy and Raritan Formations:

Fine, gray clay ............................. $15 \quad 429$

Gray silty clay .............................. 949

Hard, red clay .............................. $24 \quad 462$

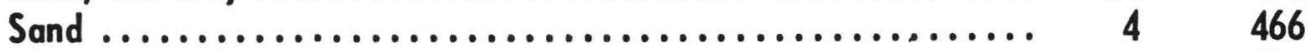

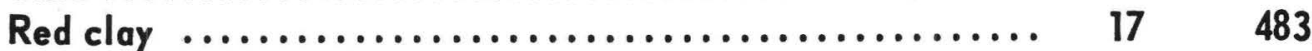

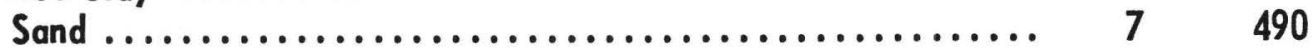

Fine to medium, variegated sand $\ldots \ldots \ldots \ldots \ldots \ldots \ldots \ldots \ldots, 31$

Red clay ................................ 1

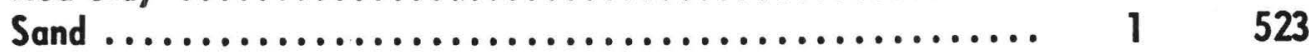


Table 3. - Selected drillers' logs of wells in Burlington County, N. J. - Continued

Well no. 171, lonac Chemical Co.

(Log by A. C. Schultes and Sons)

(Continued)

Cretaceous - Continued:

Magothy and Raritan Formations - Continued:

Thickness Depth

Hardpan .......................................

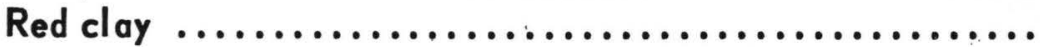

Hardpan ........................................

Sand

Red and white clay

(feef)

(feet)

Fine, gray clay

1524

$5 \quad 529$

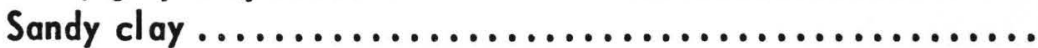

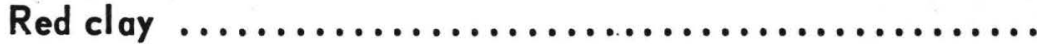

Gray sandy clay with shells

Sand

Fine, gray sand

Sand

Fine, gray sand

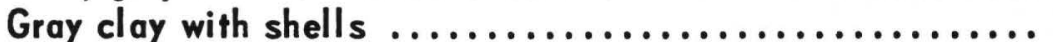

Sand

Fine, variegated sand .

Hardpan

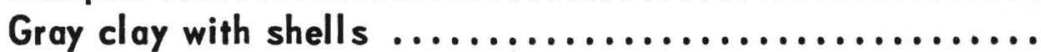

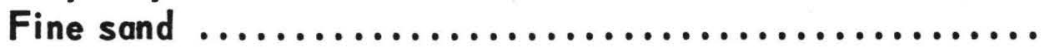

Gray clay with shells

Hardpan

Dark gray silty clay

Hardpan ............................................

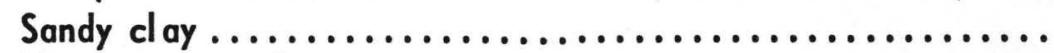

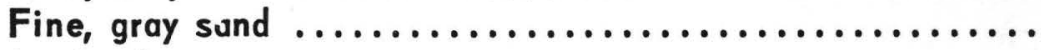

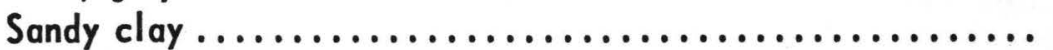

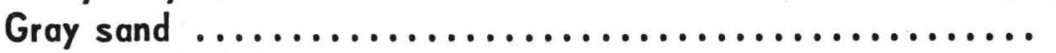

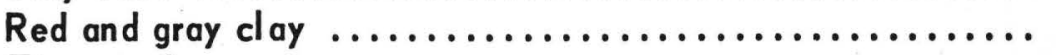

Fine sand

Red clay

Gray and red sandy clay ...........................

Sand

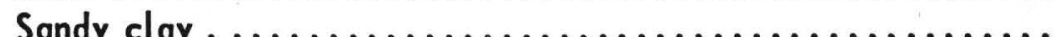

Sand

Hard, red clay .........................................

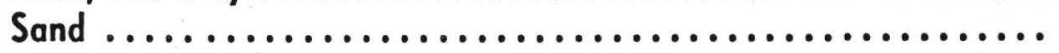

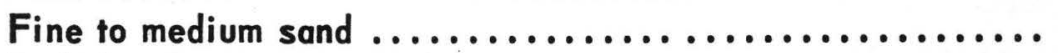

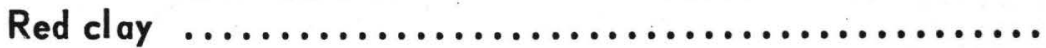

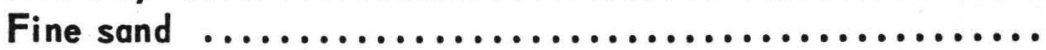

Red clay ......................................

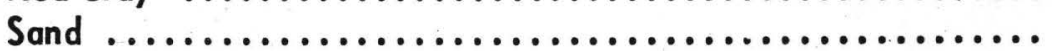

Hardpan .........................................

Red clay

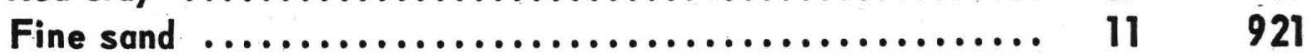


Table 3. - Selected drillers' logs of wells in Burlington County, N. J. - Continued

Well no. 172, Burlington Co. Institution No. 1

(Log by Layne-New York Co., Inc.)

Tertiary:

Altitude 59 feet

Thickness Depth

(feet) (feet)

Kirkwood Formation:

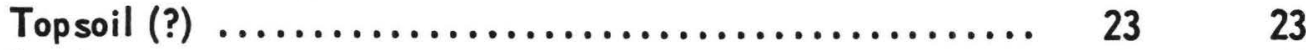

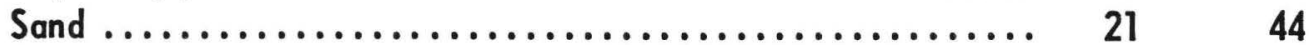

Tertiary and Cretaceous:

Manasquan and Vincentown Formations, Hornerstown Sand and

Navesink Formation (undifferentiated):

Sand and clay ................................. $22 \quad 66$

Blue clay.................................. $144 \quad 210$

Cretaceous:

Mount Laurel Sand and Wenonah Formation:

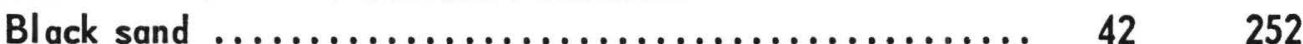

Marshalltown Formation:

Black sand and clay ........................... $107 \quad 359$

Englishtown Formation:

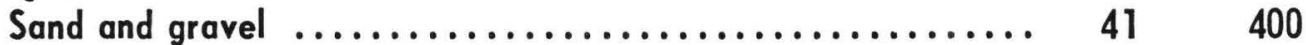

Clay ....................................... 214

Clay ........................................ $42 \quad 463$

Woodbury Clay and Merchantville Formation (undifferentiated):

Blue marl..................................... $151 \quad 614$

Magothy and Raritan Formation:

Gravel and clay ................................. $20 \quad 634$

Tough clay ................................... $41 \quad 675$

Clay ......................................... $27 \quad 702$

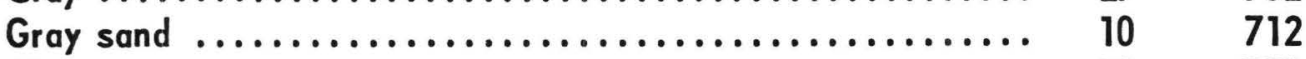

Clay ......................................... $58 \quad 770$

Early Paleozoic (?):

Wissahickon Formation:

Rock..................................... $6 \quad 776$ 
Table 3. - Selected drillers' logs of wells in Burlington County, N. J. - Continued Well no. 178, William C. Rahilly

(Log by Greenhalgh and Kaye)

Quaternary and Tertiary:

$$
\begin{gathered}
\text { Altitude } 195 \text { feet } \\
\text { Thickness } \\
\text { Depth } \\
\text { (feet) }
\end{gathered}
$$

Bridgeton Formation, Cohansey Sand, and Kirkwood Formation

(undifferentiated):

Hard, fine, yellow sand and clay $\ldots . . . . . . . . . . . . .$.

Tertiary:

Vincentown Formation and Hornerstown Sand (undifferentiated):

Yellow clay .......................................

Green marl

$11 \quad 60$

Cretaceous:

Navesink Formation:

Black marl

$40 \quad 100$

Black alay with lignite and shills....................... 22

Mount Laurel Sand and Wenonah Formation:

Green sand with shells and clay

18

158

Well no. 181, Wrightstown Municipgl Utility Authority

(Log by (unknown) 은)

Tertiary:

Altitude 120 feet

Kirkwood and Vincentown Formations (undifferentiated):

Sandy clay

Tertiary and Cretaceous:

Undifferentiated:

Sand and clay with shells $\ldots \ldots \ldots \ldots \ldots \ldots \ldots \ldots \ldots \ldots, 130 \quad 190$

Clay ........................................ $56 \quad 246$

Cretaceous:

Englishtown Formation:

Water-bearing, medium glauconitic sand ............... 8 o 254

Clayey sand $\ldots \ldots \ldots \ldots \ldots \ldots \ldots \ldots \ldots \ldots \ldots \ldots \ldots, 14,268$

8/ Richards, 1948, p. $39-76$ 
Table 3. - Selected drillers' logs of wells in Burlington County, N. J. - Continued

Well no. 183, Charles B. Hutchinson

(Log by Charles L. Mollitor Inc.)

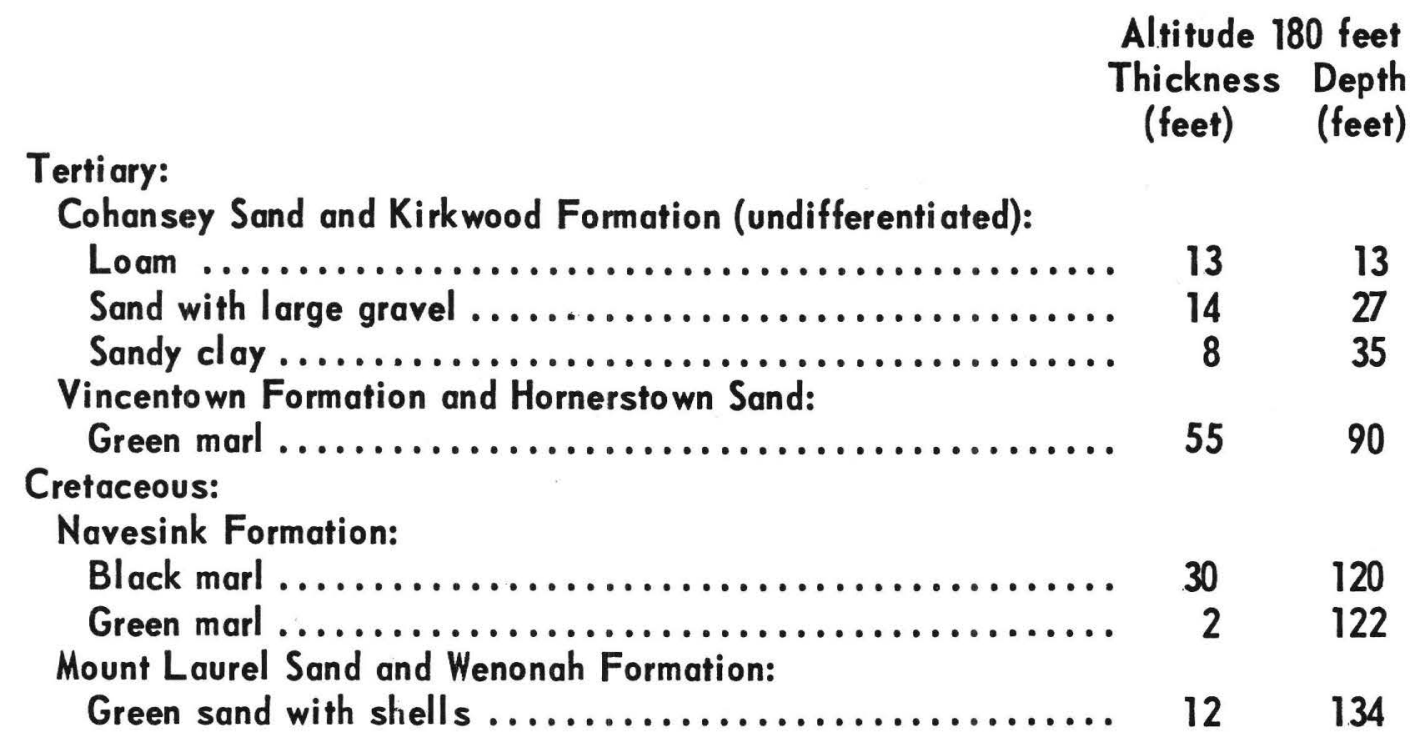


Table 3. - Selected drillers' logs of wells in Burlington County, N. J. - Continued Well no. 185, Joseph J. White Inc. (Log by Ridpath \& Potter)

Tertiary and Cretaceous:

Altitude 95 feet

Thickness Depth

(feet) (feet)

Cohansey Sand and Kirkwood Formation (undifferentiated):

Soil (?) .......................................... $15 \quad 15$

Fine, yellow sand with some coarse, angular grains ....... $10 \quad 25$

Co arse, angul ar quartz sand ......................... 5

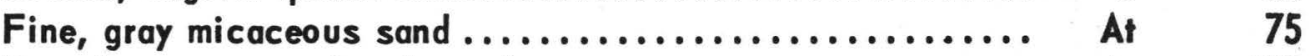

Very fine, gray sand with much mica................. At 100

Fine, dark-gray sand with some dark-brown clay ......... At 115

Manasquan and Vincentown Formations, Hornerstown Sand, and

Navesink Formation (undifferentiated):

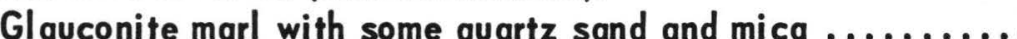

Glauconite marl ....................................

Glauconite marl with some white clay and white

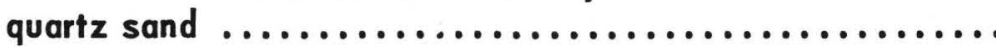

Light-green sandy clay with a little glauconite .......... A

At $\quad 132$

At 150

At $\quad 207$

Cretaceous:

Mount Laurel Sand and Wenonah Formation:

Fine, gray sand with a little lignite $\ldots . \ldots \ldots \ldots \ldots \ldots$ A

Coarse, black and white glauconitic sand with shell

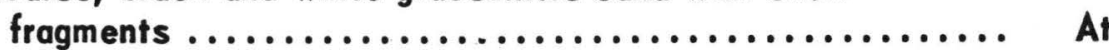

At $\quad 341$

At $\quad 380$

Marshall town Formation:

Yellowish gray glauconitic sand $\ldots \ldots \ldots \ldots \ldots \ldots \ldots \ldots . . .6$.

At $\quad 400$

Fine, gray glauconitic sand with some mica ............ At 440

Fine, gray glauconitic sand with some mica ............ At 450

Englishtown Formation:

Medium-yellow sand with some glauconite and mica ........ At 535

Medium-yellow sand with some glauconite and mica ....... $15 \quad 550$

Woodbury Clay and Merchantville Formation (undifferentiated):

Very fine, dark-gray sand with glauconite and mica......... At 700

Very fine, durk-gray sand with glauconite and mica........ $25 \quad 725$

Very fine, dark-gray sand and sandy micaceous clay....... At 730

Fine, dark-gray, sand with much glauconite and mica ...... At 800 
Table 3. - Selected drillers' logs of wells in Burlington County, N. J. - Continued Well no. 186, Joseph J. White Inc.

(Log by J. W. Jenkins)

Tertiary:

Cohansey Sand:

Fine, yellow sand

Kirkwood Formation:

Fine, gray sand with clay

Manasquan Formation:

Hard, dark-green clay .......................... 14

152

Dark-green clay with black sand .

Vincentown Formation:

Light-green clay

Tertiary and Cretaceous:

Hornerstown Sand and Navesink Formation (undifferentiated):

Dark-green clay with black sand and shells .............

Dark-brown clay with shells and black sand ...............

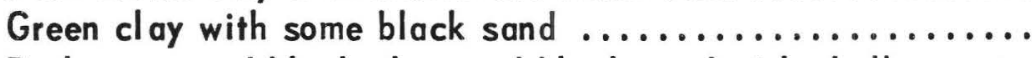

Dark-green and black clay, and black sand with shells...... Cretaceous:

Mount Laurel Sand and Wenonah Formation:

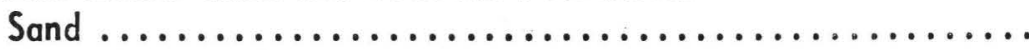

Water-bearing, fine to medium sand....

$20 \quad 365$

$11 \quad 239$

$57 \quad 296$

$24 \quad 320$

$25 \quad 345$

Well no. 196, Taunton Lakes Water Co.

(Log by J. Henry Robbins)

Tertiary:

Altitude 57 feet

Kirkwood Formation:

Yellow clay .....................................

$25 \quad 25$

Black clay

55

80

Manasquan and Vincentown (?) Formations and Hornerstown

Sand (undifferentiated):

Green clay

\section{Cretaceous:}

Navesink Formation:

Black clay

Undifferentiated:

Green clay and fine sand $\ldots \ldots \ldots \ldots \ldots \ldots \ldots \ldots, 10$

Mount Laurel Sand and Wenonah Formation:

Green and white sand 
Table 3. - Selected drillers' logs of wells in Burlington County, N. J. - Continued

Well no. 198, Riverton-Palmyra Water Company

(Log by A. C. Schultes and Sons)

\begin{tabular}{|c|c|c|}
\hline & $\begin{array}{c}\text { Altitude } 7 \\
\text { Thickness } \\
\text { (feet) }\end{array}$ & $\begin{array}{l}9 \text { fee } \\
\text { Dep } \\
\text { (fee }\end{array}$ \\
\hline $\begin{array}{l}\text { Quaternary: } \\
\text { Cape May Formation: }\end{array}$ & & \\
\hline Fine, brown sand ...... & 4 & \\
\hline Fine, brown clayey sand. & 2 & \\
\hline ............................ & 6 & \\
\hline retaceous: & & \\
\hline Merchantville Formation: & & \\
\hline $\begin{array}{l}\text { Clay } . \ldots \ldots \ldots \ldots \ldots \ldots \ldots \ldots \ldots \ldots \\
\text { Magothy and Raritan Formations: }\end{array}$ & 7 & \\
\hline Fine to medium, brown sand ........... & 6 & \\
\hline Medium to coarse, brown sand $\ldots \ldots \ldots \ldots \ldots \ldots \ldots \ldots \ldots$ & 5 & \\
\hline 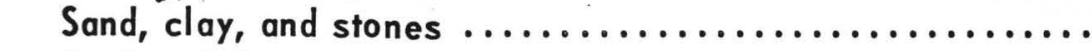 & 7 & \\
\hline Sand and clay $\ldots \ldots \ldots \ldots \ldots \ldots \ldots \ldots \ldots \ldots \ldots \ldots \ldots \ldots \ldots \ldots$ & 2 & \\
\hline 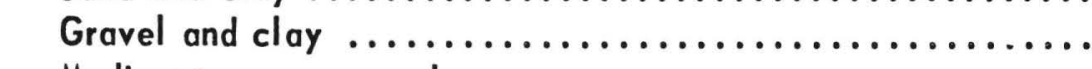 & 1 & \\
\hline Medium to coarse sand $\ldots \ldots \ldots$. & 4 & \\
\hline 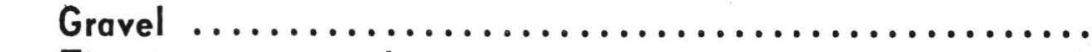 & 2 & \\
\hline 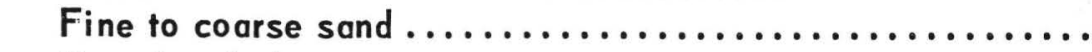 & 4 & \\
\hline Gravel and clay $, \ldots, \ldots, \ldots, \ldots, \ldots, \ldots, \ldots, \ldots, \ldots, \ldots$ & 4 & \\
\hline 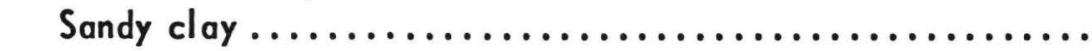 & 5 & \\
\hline (n..., & 4 & \\
\hline nd and gravel $\ldots \ldots \ldots \ldots \ldots \ldots \ldots \ldots$ & 2 & \\
\hline 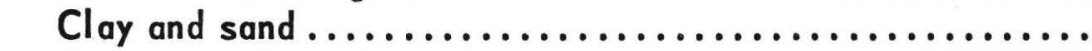 & 11 & \\
\hline Medium sand $[\ldots, \ldots, \ldots, \ldots, \ldots, \ldots, \ldots, \ldots, \ldots, \ldots, \ldots$ & 1 & \\
\hline 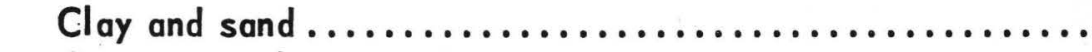 & 8 & \\
\hline vel and sand $[\ldots \ldots \ldots \ldots, \ldots, \ldots, \ldots, \ldots, \ldots$ & 3 & \\
\hline 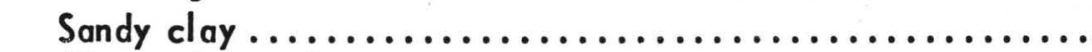 & 10 & \\
\hline & 4 & \\
\hline Clay and sand & 4 & \\
\hline 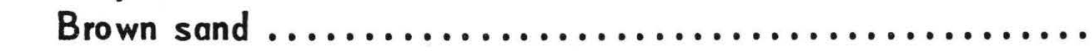 & 12 & \\
\hline Coarse gravel $\ldots \ldots \ldots \ldots \ldots \ldots \ldots \ldots \ldots \ldots \ldots \ldots \ldots \ldots$ & 5 & \\
\hline & 1 & \\
\hline and gravel $\ldots . . . \ldots$. & 25 & \\
\hline Sand and clay $\ldots \ldots \ldots, \ldots, \ldots, \ldots, \ldots, \ldots, \ldots, \ldots, \ldots, \ldots$ & 6 & \\
\hline 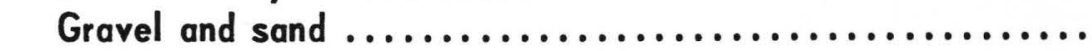 & 5 & \\
\hline Sandy clay $[\ldots, \ldots, \ldots, \ldots, \ldots, \ldots, \ldots, \ldots, \ldots, \ldots, \ldots, \ldots$ & 5 & \\
\hline 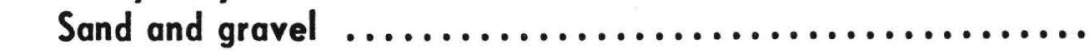 & 14 & \\
\hline Gravel and sand $\ldots \ldots \ldots \ldots \ldots \ldots \ldots \ldots \ldots \ldots \ldots \ldots \ldots \ldots$ & 9 & \\
\hline Gravel and clay $\ldots \ldots \ldots \ldots \ldots \ldots \ldots \ldots \ldots \ldots \ldots \ldots \ldots \ldots \ldots \ldots$ & 2 & \\
\hline 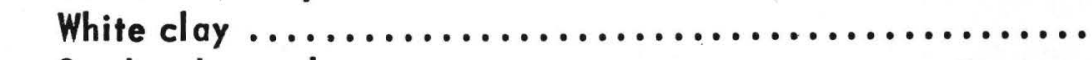 & 4 & \\
\hline${ } \ldots \ldots, \ldots, \ldots, \ldots, \ldots, \ldots, \ldots$ & 6 & \\
\hline
\end{tabular}

(Continued) 
Table 3. - Selected drillers' logs of wells in Burlington County, N. J. - Continued

\section{Well no. 198, Riverton-Palmyra Water Company}

(Log by A. C. Schultes and Sons)

(Continued)

Cretaceous - Continued:

Magothy and Raritan Formations - Continued:

Thickness Depth

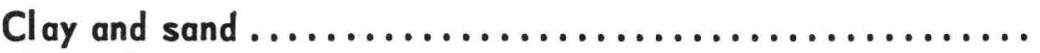

(feet) (feet)

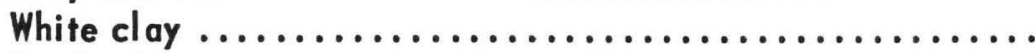

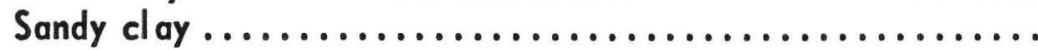

1218

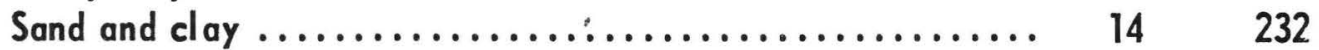

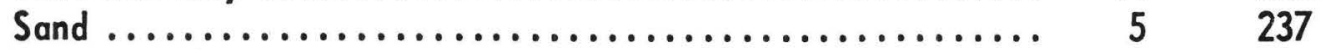

Sand and clay .................................. 12

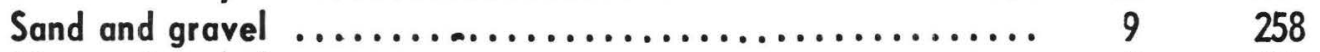

Clay and gravel ............................ 1259

Sand and gravel ............................. 6265

Medium to coarse, brown sand with some gravel .......... 6271

Medium to coarse, brown sand and gravel ............. $11 \quad 282$

Red clay .................................. 55287

Very hard, brown and white clay ................... 2289

Coarse gravel ................................ $13 \quad 302$

Early Paleozoic (?):

Wissahickon Formation:

Stones, gravel, and weathered, micaceous rock ......... $2 \quad 304$

Rock with some sand ....................... 2306

Weathered rock ............................. 308 
Table 3. - Selected drillers' logs of wells in Burlington County, N. J. - Continued Well no. 199, Moorestown Township Water Department (Log by Layne-New York Co., Inc.)

Altitude 59 feet Thickness Depth (feet) (feet)

Cretaceous:

Englishtown Formation:

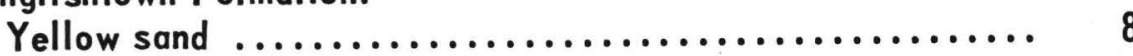

$8 \quad 8$

Undifferentiated:

Gray clay with hard streaks

$84 \quad 92$

Magothy and Raritan Formations:

Gray clay

$48 \quad 140$

Red, white, and gray clay......................... $10 \quad 150$

Gray clay with hard streaks...$\ldots \ldots \ldots \ldots \ldots \ldots \ldots \ldots$. $70 \quad 220$

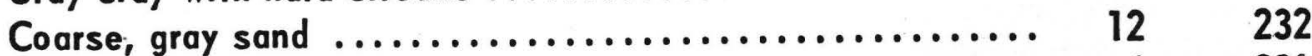

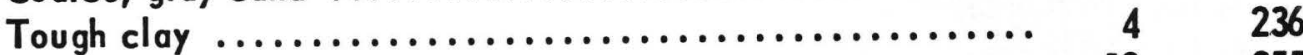

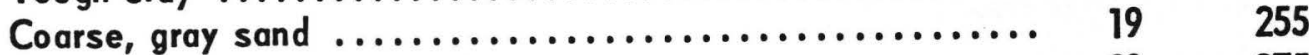

Coarse, gray clayey sand $\ldots \ldots \ldots \ldots \ldots \ldots \ldots \ldots \ldots \ldots, 20,275$

Medium to coarse sand with pebbles.................. $68 \quad 343$

Red and white variegated clay .................... $17 \quad 360$

Well no. 201, Cedar Bridge Fire Tower

(Log by J. Henry Robbins)

Tertiary:

Altitude 200 feet

Cohansey Sand:

Yellow clayey sand with small pebbles ................. 30 30

Layers of light-gray to buff clay and sand ............... $30 \quad 60$

Fine to coarse, yellow clayey sand with small

pebbles...................................... $10 \quad 70$

Fine to coarse, yellow clayey sand $\ldots \ldots \ldots \ldots \ldots \ldots \ldots ., 30 \quad 100$

Light-gray clay with thin sand layers................ 10 110 
Table 3. - Selected drillers' logs of wells in Burlington County, N. J. - Continued

Well no. 202, Inductotherm Corporation

(Lọ by Charles L. Mollitor Inc.)

Cretaceous:

Englishtown Formation:

Clay ..........................................

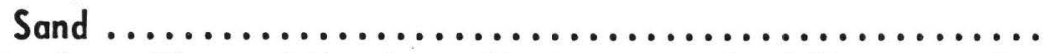

Woodbury Clay and Merchantville Formation (undifferentiated):

Gray marl .......................................

Black clay.................................. 58

Green marl .................................. 70

Magothy and Raritan Formations:

Gray sandy clay ...............................

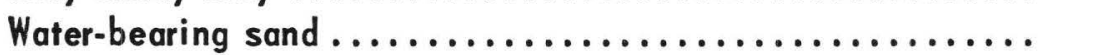

Red clay..................................... 16

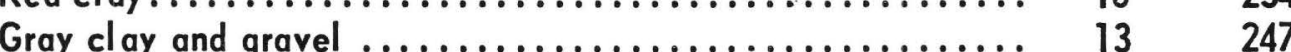

White clay ..................................... 1248

Black clay and gravel ........................... $36 \quad 284$

White clay ................................... $6 \quad 290$

Black clay.................................... $15 \quad 305$

White clay .................................... 4309

Red clay .................................... $16 \quad 325$

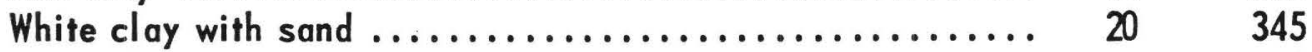

White clay .................................... 11356

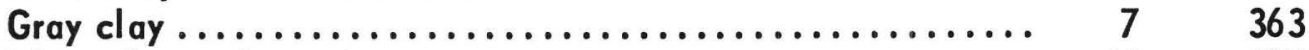

White clay with sand ................................ $22 \quad 385$

Water-bearing sand $\ldots \ldots \ldots \ldots \ldots \ldots \ldots \ldots \ldots \ldots \ldots, 138 \ldots \ldots$

Gray clay ..................................... 3389

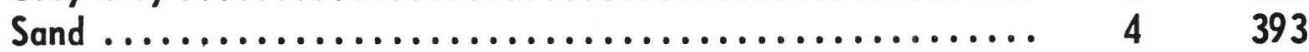

Clay ....................................... $12 \quad 405$

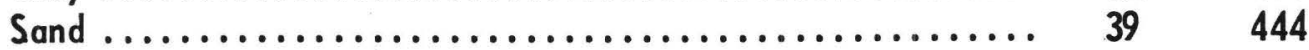

Gray clay ................................... 194 
Table 3. - Selected drillers' logs of wells in Burlington County, N. J. - Continued Well no. 208, Mount Laurel Water Company (Log by A. C. Schultes and Sons)

Quaternary:

Altitude 30 feet Thickness

Depth

(feet)

(feet)

Cape May Formation:

Topsoil, sand, and gravel

Cretaceous:

Marshalltown Formation:

Gray sandy clay

Englishtown Formation:

Fine, gray sand

Woodbury Clay:

Gray clay and fine, hard sand.

Merchantville Formation:

Black marl

192

Magothy and Raritan Formations:

Fine to medium sand ......................... 24

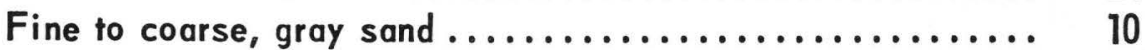

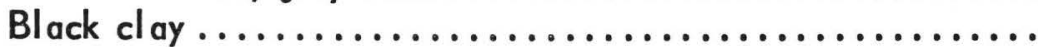

Fine to coarse, gray sand $\ldots \ldots \ldots \ldots \ldots \ldots \ldots \ldots \ldots \ldots$

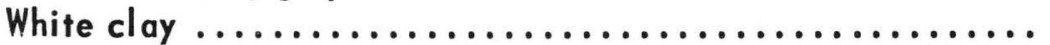

Fine to coarse gray sand and gravel .

Clay

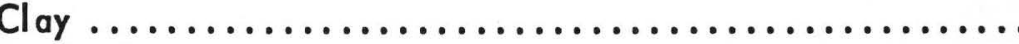

Sandy clay

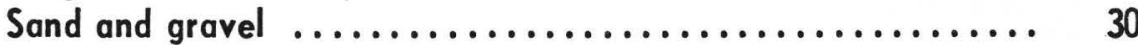

Red clay .................................. 5

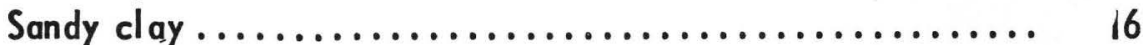

Coarse sand and gravel ......................... 13

Red clay

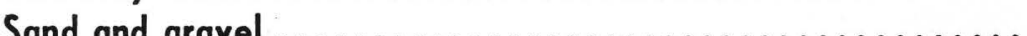

Red clay

Sand, gravel, and clay ......................... 8

Coarse sand and gravel ........................ 38

Red clay ....................................

Coarse sand and gravel ........................ 21

Red clay

Coarse sand and gravel

Early Paleozoic (?):

Wissahickon Formation:

Weathered rock 


\begin{tabular}{|c|c|c|c|c|c|c|c|c|c|c|c|c|c|c|c|c|c|c|c|c|c|}
\hline \multirow[t]{2}{*}{$=0$} & \multirow{2}{*}{$\begin{array}{l}\text { Date of } \\
\text { oalloction }\end{array}$} & \multirow{2}{*}{$\begin{array}{l}\text { Tom- } \\
\text { Purer } \\
\text { tors) }\end{array}$} & \multirow[t]{2}{*}{$\begin{array}{l}\mathrm{s1110 \textrm {oa }} \\
(\mathrm{S10})\end{array}$} & \multirow{2}{*}{$\begin{array}{l}\text { Total } \\
\text { 1ron } \\
\text { (Fo) }\end{array}$} & \multirow{2}{*}{ 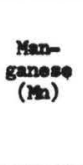 } & \multirow{2}{*}{ 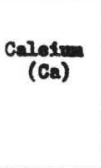 } & \multirow{2}{*}{$\begin{array}{c}\text { Mage- } \\
\text { neavivim } \\
\text { (Mg) }\end{array}$} & \multirow{2}{*}{$\begin{array}{c}\text { Sodium } \\
(\mathrm{Na})\end{array}$} & \multirow{2}{*}{$\begin{array}{l}\text { Potas- } \\
\text { stum } \\
\text { (I) }\end{array}$} & \multirow{2}{*}{ 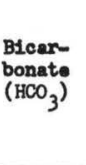 } & \multirow{2}{*}{$\begin{array}{c}\text { Sulfate } \\
\left(\mathrm{sO}_{4}\right)\end{array}$} & \multirow{2}{*}{$\begin{array}{l}\text { Chloride } \\
\text { (C1) }\end{array}$} & \multirow{2}{*}{$\begin{array}{l}\text { Fluo- } \\
\text { rlde } \\
\text { (F) }\end{array}$} & \multirow{2}{*}{$\begin{array}{l}\mathrm{N}_{1} 1 \mathrm{tratat} \\
\left(\mathrm{NO}_{3}\right)\end{array}$} & \multirow{2}{*}{ 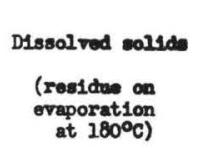 } & \multicolumn{2}{|c|}{$\begin{array}{l}\text { Hardness } \\
\text { es } \mathrm{CaCO}_{3}\end{array}$} & \multirow{2}{*}{$\begin{array}{l}\text { Specific } \\
\text { conduct- } \\
\text { ance } \\
\text { (mitcro- } \\
\text { mhos at } \\
25^{\circ} \mathrm{C} \text { ) }\end{array}$} & $\mathrm{nH}$ & Source & Map \\
\hline & & & & & & & & & & & & & & & & $\begin{array}{c}\text { calcium } \\
\text { mag- } \\
\text { nessium }\end{array}$ & $\begin{array}{l}\text { Moncar- } \\
\text { bonato }\end{array}$ & & pin & dats & \\
\hline 3 & $5-21-51$ & 55 & 10 & 0,01 & 3.6 & 23 & 10 & 14 & 10 & 80 & 38 & 20 & 0.2 & 5.9 & 203 & 98 & 33 & 318 & 7.2 & usas & 3 \\
\hline 5 & $9-9-52$ & - & -- & .10 & .00 & ---- & - & - & - & 12 & ---- & 5.0 & .0 & -- & 54 & 28 & 18 & -- & 5.7 & D & 5 \\
\hline 6 & $5-22-51$ & 57 & 8.4 & .02 & .00 & .28 & 4.5 & 2.6 & 5.5 & 103 & 12 & 1.2 & .1 & .5 & 113 & 88 & 4 & 190 & 7.6 & uscs & 6 \\
\hline 7 & $5-31-61$ & - & - & 15 & .18 & -- & -- & - & - & 52 & --- & --- & - & -- & -- & 38 & 0 & -- & 6.9 & NJगH & 7 \\
\hline 8 & $9-310-54$ & 56 & - & .06 & -- & --- & $\ldots$ & $u_{4}$ & & 35 & 42 & 9.8 & --- & 16 & - & 68 & 39 & 206 & 6.3 & usas & 8 \\
\hline 11 & $3-22-51$ & 56 & 27 & .17 & .10 & 43 & 4.0 & 2.2 & 5.7 & 149 & 7.0 & 2.6 & .4 & .3 & 166 & 224 & 2 & 251 & 8.2 & vsas & 11 \\
\hline 13 & $5-3-51$ & 55 & 12 & .00 &.$\infty$ & 8.0 & 4.4 & 4.8 & 1.4 & 8 & 14 & 8.0 & .0 & 21 & 84 & 38 & 32 & 112 & 7.1 & usGS & 13 \\
\hline 24 & $6-19-59$ & -- & 9.8 & 2.4 & .05 & 16 & 2.8 & 9. & & 68 & 12 & 1.8 & .1 & .2 & 105 & 52 & 0 & 137 & 6.5 & usas & $\mu_{4}$ \\
\hline 25 & $6-19-59$ & -- & 11 & 2.2 & .04 & 16 & 3.3 & 8. & & 68 & 11 & 2.8 & .1 & .2 & 85 & 54 & 0 & مالע & 6.8 & usGs & 15 \\
\hline 16 & $6-18-59$ & - & 9.8 & 2.1 & .09 & 14 & 4.7 & 6. & & 66 & 12 & 1.4 & .1 & .4 & 100 & 55 & 1 & 134 & 6.4 & uscs & 16 \\
\hline 18 & $3-1-61$ & --- & 7.8 & 2.0 & .06 & 15 & 2.1 & 8.2 & 3.8 & 64 & 8.2 & 5.1 & .1 & .4 & 84 & 46 & 0 & 139 & 7.1 & usas & 18 \\
\hline 19 & $3-1-61$ & -- & 8.3 & 2.8 & .09 & 16 & 2.6 & 6.0 & 3.8 & 67 & 8.2 & 2.5 & .0 & .8 & 82 & 51 & 0 & 233 & 6.8 & usas & 19 \\
\hline 20 & 3- 1-61 & --- & 9.8 & 3.1 & .08 & 17 & 2.6 & 6.0 & 3.8 & 69 & 7.6 & 2.4 & .0 & .5 & 84 & 53 & 0 & 135 & 6.9 & usas & 20 \\
\hline 21 & $5-22-51$ & 56 & $\underline{\mu}$ & .22 & .00 & 26 & 2.9 & 2.8 & 3.9 & 92 & 7.0 & 2.0 & .1 & .2 & 100 & 77 & 1 & 167 & 8.3 & USGS & 21 \\
\hline 23 & $4-18-58$ & -- & --- & 9.3 & .35 & 18 & 6.8 & --- & --- & 56 & --- & 6.9 & .2 & -- & 104 & 73 & 27 & -- & 6.2 & 0 & 23 \\
\hline 24 & 6-13-61 & 56 & 10 & 5.2 & .05 & 3.3 & 1.7 & 2.2 & 1.0 & 16 & 5.5 & 2.2 & .0 & .2 & 40 & 15 & 2 & 47 & 6.1 & USGS & 24 \\
\hline 28 & $6-b 511$ & 60 & 9.6 & 1.1 & .00 & 28 & 5.2 & 3.5 & 6.8 & 105 & 20 & 1.2 & .0 & 2.4 & 129 & 92 & 5 & 216 & 7.3 & USGS & 28 \\
\hline 32 & $5-3-51$ & 58 & 11 & - & .13 & 19 & 3.2 & 4.5 & 4.1 & 47 & 32 & 2.4 & .1 & .4 & 94 & 61 & 22 & 254 & 6.6 & usGs & 32 \\
\hline 33 & $11-9=54$ & - & --- & .83 & .00 & 75 & 17 & --- & --- & 95 & ---- & 2.0 & --- & - & -- & 258 & 180 & - & 7.2 & 0 & 33 \\
\hline 35 & $5-28-51$ & 56 & 16 & .35 & .00 & $\psi$ & 3.0 & 2.2 & 4.3 & 153 & 5.0 & 3.4 & .1 & .3 & 156 & 122 & 0 & 253 & 7.8 & usGs & 35 \\
\hline 36 & $5-40-52$ & 59 & 11 & .30 & .00 & 21 & 5.4 & 3.3 & 8.4 & 107 & 4.5 & 2.2 & .0 & .9 & 109 & 75 & 0 & 187 & 7.7 & USGS & 36 \\
\hline 38 & 1960 & - & -- & .08 & .00 & $\ldots$ & - & - & --. & 113 & $-\ldots$ & 2.0 & --- & - & 122 & 82 & 0 & - & 8.0 & D & 38 \\
\hline 40 & 1960 & - & - & -- & .04 & - & -- & $\ldots$ & $\ldots$ & 15 & ---- & 9.0 & --- & - & --- & 52 & 40 & -- & 5.6 & 0 & 40 \\
\hline bl & $1-10-61$ & - & - & .04 & - & -- & -- & - & --- & 100 & --- & 3.0 & .0 & --- & - & 80 & 0 & -- & 7.8 & NJDH & 41 \\
\hline 15 & $5-3-51$ & 55 & 12 & .71 & -- & 3.6 & 1.5 & 2.8 & .5 & 3 & 14 & 3.2 & .0 & .8 & 41 & 15 & 13 & 49 & 5.6 & usas & 45 \\
\hline 47 & $5-2 h-51$ & - & - & 2.0 & - & 23 & 10 & 14 & 10 & 88 & 38 & 20 & - & 5.9 & -- & 98 & 26 & -- & 7.2 & 0 & 47 \\
\hline 50 & $112-5-54$ & - & - & 2.1 &.$\infty$ & 41 & 13 & ---- & --- & 98 & -- & 2.0 & - & - & - & 156. & 76 & - & 7.2 & 0 & 50 \\
\hline 61 & $8-14-52$ & 56 & 26 & .10 &.$\infty$ & .8 & .9 & 2.9 & 2.2 & 1 & 10 & 3.1 & .0 & .1 & 49 & 6 & 5 & 49 & 4.7 & USGS & 61 \\
\hline 66 & 6-13-61 & 75 & 18 & .60 & .03 & 39 & 2.9 & 2.0 & 6.0 & 138 & 5.9 & 2.8 & .1 & .4 & 24.5 & 110 & 0 & 232 & 7.7 & USGS & 66 \\
\hline 67 & $6-13-61$ & 62 & 9.3 & 10 & .14 & 17 & 2.8 & 2.5 & 2.5 & 60 & 11 & 2.6 & .1 & .1 & 77 & 54 & 5 & 128 & 6.5 & usGS & 67 \\
\hline 73 & $11-20-57$ & - & - & & - & 33 & 3.9 & 7.0 & - & 113 & 23 & 3.0 & - & - & -- & 110 & 18 & - & 7.9 & D & 73 \\
\hline$\pi 7$ & $12-6-54$ & -- & - & .06 & .00 & 12 & 25 & - & $-\ldots$ & 1 & -- & 9.0 & -- & - & - & $\$ 2$ & 91 & - & 5.9 & 0 & 77 \\
\hline$\infty$ & 12- $9-58$ & -- & - & 4.5 & .00 & 18.4 & 3.4 & 7.0 & -- & 57 & 19 & 4.0 & -- & - & - & 60 & 14 & -- & 7.4 & D & 89 \\
\hline 97 & $6-13-61$ & 65 & $2 \mathrm{~h}$ & 3.7 & .06 & 59 & 1.3 & 1.8 & 2.5 & 168 & 18 & 4.0 & .4 & .2 & 205 & 153 & 15 & 305 & 7.3 & usas & 97 \\
\hline 120 & $10-10-57$ & 63 & 13 & .65 & .02 & 38 & 5.6 & 2.0 & 6.7 & 151 & 3.5 & 2.6 & .4 & .2 & 141 & 118 & 0 & 239 & 8.2 & usas & 118 \\
\hline $1 \mathrm{n}$ & $6-23-61$ & 62 & 8,8 & .72 & .02 & 29 & 6.4 & 5.5 & 13 & m & 25 & 1.6 & .2 & 1.0 & 160 & 99 & 8 & 234 & 7.5 & UISGS & 124 \\
\hline 125 & $20.10-57$ & 6 & 17 & .87 & .06 & 34 & 3.2 & 1.6 & 4.1 & 117 & 7.7 & 2.4 & .4 & .0 & 139 & 98 & 2 & 205 & 7.3 & USGS & 125 \\
\hline 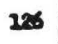 & $1-4-60$ & -1 & - & 16 & - & 22 & 4 & -- & - & 46 & 2.8 & 2.0 & - & - & - & 46 & 8 & --- & 6.3 & 0 & 126 \\
\hline
\end{tabular}


Table 4.--Chemical analyses, in parts per million, of water from wells in Burlington County, N. J.--Continued

\begin{tabular}{|c|c|c|c|c|c|c|c|c|c|c|c|c|c|c|c|c|c|c|c|c|c|}
\hline \multirow{2}{*}{$\begin{array}{l}\text { Map } \\
\text { no. }\end{array}$} & \multirow{2}{*}{$\begin{array}{l}\text { Date of } \\
\text { collection }\end{array}$} & \multirow{2}{*}{$\begin{array}{l}\text { Tem- } \\
\text { pera- } \\
\text { ture } \\
\left({ }^{\circ}\right)\end{array}$} & \multirow{2}{*}{$\begin{array}{l}\text { Silica } \\
\left(\mathrm{SiO}_{2}\right)\end{array}$} & \multirow{2}{*}{$\begin{array}{l}\text { Total } \\
\text { iron } \\
(\mathrm{Fe})\end{array}$} & \multirow{2}{*}{$\begin{array}{l}\text { Man- } \\
\text { ganese } \\
(\mathrm{Mn})\end{array}$} & \multirow{2}{*}{$\frac{\text { Calcium }}{(\mathrm{Ca})}$} & \multirow{2}{*}{$\begin{array}{c}\text { Mag- } \\
\text { nesium } \\
\text { (1/g) }\end{array}$} & \multirow{2}{*}{$\begin{array}{c}\text { Sodium } \\
(\mathrm{Na})\end{array}$} & \multirow{2}{*}{$\begin{array}{l}\text { Potas- } \\
\text { sium } \\
(\mathrm{K})\end{array}$} & \multirow{2}{*}{$\begin{array}{l}\text { Bicar- } \\
\text { bonate } \\
\left(\mathrm{HCO}_{3}\right)\end{array}$} & \multirow{2}{*}{$\begin{array}{l}\text { Sulf ate } \\
\left(\mathrm{SO}_{4}\right)\end{array}$} & \multirow{2}{*}{$\begin{array}{c}\text { Chloride } \\
\text { (CI) }\end{array}$} & \multirow{2}{*}{$\begin{array}{l}\text { Fluo- } \\
\text { ride } \\
\text { (F) }\end{array}$} & \multirow{2}{*}{$\begin{array}{c}\text { Nitrate } \\
\left(\mathrm{NO}_{3}\right)\end{array}$} & \multirow{2}{*}{$\begin{array}{l}\text { Dissolved solids } \\
\text { (residue on } \\
\text { evaporation } \\
\text { at } 180^{\circ} \mathrm{C} \text { ) }\end{array}$} & \multicolumn{2}{|c|}{$\begin{array}{l}\text { Hardness } \\
\text { as } \mathrm{CaCO}_{3}\end{array}$} & \multirow{2}{*}{$\begin{array}{l}\text { Specific } \\
\text { conduct- } \\
\text { ance } \\
\text { (micro- } \\
\text { mhos at } \\
25^{\circ} \mathrm{C} \text { ) }\end{array}$} & \multirow{2}{*}{$\mathrm{pH}$} & \multirow{2}{*}{$\begin{array}{c}\text { Source } \\
\text { of } \\
\text { date }\end{array}$} & \multirow{2}{*}{$\begin{array}{l}\text { Map } \\
\text { no. }\end{array}$} \\
\hline & & & & & & & & & & & & & & & & $\begin{array}{c}\text { Calcium } \\
\text { mag- } \\
\text { nesium }\end{array}$ & $\begin{array}{l}\text { Noncar- } \\
\text { bonate }\end{array}$ & & & & \\
\hline 131 & $6-13-61$ & 70 & 16 & .50 & 0.02 & 36 & 2.6 & 1.8 & 3.2 & .20 & 5.0 & 3.2 & 0.1 & 0.2 & 140 & 101 & 2 & 203 & 7.8 & USOS & 131 \\
\hline 136 & $7-20-59$ & - & $\cdots$ & -- & .00 & 5.0 & 3.0 & --- & ---- & 2 & 15 & 5.0 & -- & --- & -- & 24 & 23 & --- & 5.8 & 0 & 136 \\
\hline 149 & $6-13-61$ & 60 & 32 & 11 & .21 & 27 & 3.2 & 2.5 & 4.8 & 34 & 18 & 31 & .2 & .4 & 170 & 81 & 53 & 210 & 6.0 & usas & $\Perp_{19}$ \\
\hline 158 & $1-7-61$ & $\cdots$ & $\cdots$ & .16 & .00 & -- & -- & -.-- & --- & 142 & ---- & 6.0 & .2 & .2 & 136 & 70 & 0 & - & 8.1 & NJDH & 158 \\
\hline 168 & $21-8-54$ & --- & - & .02 & .00 & 75 & 18 & $-\cdots$ & ---- & 109 & --- & 4.0 & -- & --- & $\cdots$ & 260 & 171 & - & 7.2 & 0 & 168 \\
\hline 171 & $4-27-60$ & -- & 9.0 & .70 & .00 & 20 & 4.9 & 12 & -- & 87 & 7.0 & 6.0 & -- & --- & -- & 70 & 0 & -- & 8.1 & 0 & 171 \\
\hline 172 & $4-23-56$ & 58 & 9.7 & .24 & .01 & 27 & 4.6 & 2.7 & 7.4 & 112 & 6.4 & 2.2 & 0.1 & 0.9 & 218 & 86 & 0 & 196 & 8.0 & USGS & 172 \\
\hline 173 & 10- 9-57 & 59 & 31 & 9.8 & .11 & 19 & 1.1 & 1.6 & 4.3 & 47 & 18 & 3.5 & .8 & .4 & 116 & 52 & I4 & 129 & 6.5 & USGS & 173 \\
\hline 174 & $5-2 L-51$ & 58 & 9.8 & .68 & .45 & 26 & 4.6 & 3.2 & 6.3 & 98 & 15 & 1.2 & .1 & .6 & $11:$ & 86 & 3 & 190 & 7.9 & USGS & 174 \\
\hline 175 & $10--55$ & ---- & --- & .15 & .01 & --- & -- & --- & --- & 11 & -- & 11 & --- & --- & 84 & 50 & 41 & -- & 6.2 & 0 & 175 \\
\hline 176 & $6-13-61$ & 61 & 45 & 1.9 & .05 & 33 & 3.5 & 2.5 & 2.5 & .108 & 8.4 & 3.0 & .5 & .4 & $15 \mathrm{~L}$ & 97 & 9 & 197 & 6.8 & USGS & 176 \\
\hline 177 & $6-13-61$ & 64 & 32 & 7.2 & .09 & 36 & 3.0 & 2.0 & 7.0 & 62 & 28 & 25 & .6 & .0 & 180 & 103 & 52 & 247 & 6.9 & USGS & 177 \\
\hline 181 & $5-28-51$ & 55 & 21 & .64 & .12 & 37 & 1.8 & 2.0 & 3.3 & 121 & 8.4 & 2.5 & .1 & .2 & 139 & 100 & 1 & 224 & 8.0 & USGS & 181 \\
\hline 184 & $6-17-59$ & $\cdots$ & 9.0 & .64 & .03 & 23 & 4.6 & \multicolumn{2}{|c|}{8.0} & 104 & 7.1 & .8 & .0 & .4 & 150 & 77 & 0 & 176 & 7.1 & USGS & 184 \\
\hline 188 & $5-2-51$ & 59 & 6.0 & .00 & .00 & 5.2 & .0 & 5.0 & .3 & 10 & .0 & 3.2 & 1.9 & 1.9 & 4 & 13 & 5 & 46 & 6.9 & USGS & 188 \\
\hline 189 & $8-14-51$ & 56 & 4.3 & .12 & .00 & 1.3 & 1.9 & 2.4 & .7 & 3 & 4.5 & 8.2 & .0 & .3 & 26 & 11 & 9 & 79 & 5.0 & USGS & 189 \\
\hline 190 & $6-21-51$ & 65 & 6.1 & 10 & .52 & 2.4 & .4 & 3.0 & .9 & 5 & 4.2 & 5.0 & .1 & .2 & 25 & 8 & 4 & 35 & 5.5 & USGS & 190 \\
\hline 191 & $8-1 ل_{4}-51$ & 56 & 32 & .32 & .00 & 1.0 & .8 & 2.5 & 2.0 & 2 & 7.0 & 3.6 & .0 & .2 & 54 & 6 & 4 & 47 & 4.7 & USGS & 191 \\
\hline 192 & $8-14-51$ & 59 & 5.2 & .04 & .00 & .2 & .7 & 2.6 & .8 & 3 & .0 & 4.2 & .0 & 2.1 & 16 & 3 & 1 & 23 & 5.5 & USGS & 192 \\
\hline 193 & $6-21-51$ & 58 & 2.7 & 5.7 & .38 & 1.2 & 1.6 & 3.1 & .6 & 0 & 7.5 & 8.5 & .0 & .3 & 29 & 10 & 10 & 63 & 404 & USGS & 193 \\
\hline 194 & $6-21-51$ & 57 & 13 & .28 & .00 & 22 & 5.7 & 5.3 & 8.5 & 111 & 5.0 & 2.0 & .1 & .0 & 1115 & 78 & 0 & 192 & 8.1 & USGS & 194 \\
\hline 195 & $10-9-57$ & 55 & 20 & 11 & .20 & 5.9 & 1.0 & 1.6 & 1.8 & $I_{4}$ & 10 & 2.3 & .7 & .4 & 50 & 19 & 7 & 57 & 6.1 & USGS & 195 \\
\hline 196 & $6-21-51$ & 58 & 15 & .19 & .00 & 26 & 2.4 & 2.7 & 3.7 & 93 & 6.5 & 1.8 & .1 & .2 & 106 & 75 & 0 & 165 & 8.0 & USGS & 196 \\
\hline 198 & $9-10-59$ & $-\cdots$ & 12 & .10 & .00 & $\cdots$ & --- & 4.0 & ---- & 7 & .0 & 5.0 & .1 & --- & -- & 8 & 2 & -- & 6.6 & D & 198 \\
\hline 203 & $6-13-61$ & 69 & 1.5 & .47 & .04 & 1.6 & 1.0 & 1.8 & .5 & 2 & 6.7 & 2.2 & .1 & .2 & 23 & 8 & 7 & 33 & 4.8 & USGS & 203 \\
\hline 204 & $6-13-61$ & 56 & 13 & 11 & .40 & 13 & 5.0 & 4.4 & 3.0 & 18 & 28 & 7.6 & .2 & .2 & 125 & 53 & 24 & 197 & 5.8 & USGS & 204 \\
\hline 205 & $6-13-61$ & 57 & 14 & .25 & .10 & 32 & 2.1 & 2.0 & 3.0 & 106 & 4.6 & 2.8 & .4 & .2 & 120 & 89 & 2 & 184 & 7.7 & USGS & 205 \\
\hline 206 & $6-13-61$ & 59 & 10 & 1.2 & $: 03$ & 33 & 8.9 & 2.5 & 7.5 & 2118 & 28 & 1.2 & .1 & .2 & 160 & 119 & 23 & 257 & 7.6 & osas & 206 \\
\hline 207 & $6-13-61$ & 70 & 7.8 & 11 & .29 & 6.5 & 3.3 & 1.8 & 3.2 & 4 & 21 & 7.6 & .3 & .2 & 73 & 30 & 26 & 92 & 5.1 & USGS & 207 \\
\hline
\end{tabular}

